\title{
DISCOVERING DISEASE CAUSING VARIANTS IN DOGS THROUGH WHOLE GENOME SEQUENCING
}

\begin{abstract}
A Dissertation presented to the Faculty of the Graduate School
University of Missouri
\end{abstract}

\section{In Partial Fulfillment}

Of the Requirements for the Degree

Doctor of Philosophy in Veterinary Pathobiology

by

Ana Leticia Kolicheski

Dr. Gary S. Johnson, Dissertation Supervisor

July 2017 
The undersigned, appointed by the Dean of the Graduate School, have examined the dissertation entitled DISCOVERING DISEASE CAUSING VARIANTS IN DOGS THROUGH WHOLE GENOME SEQUENCING, presented by Ana Leticia Kolicheski a candidate for the degree of Doctor of Philosophy in Pathobiology and hereby certify that in their opinion it is worthy of acceptance.

\begin{tabular}{l}
\hline Dr. Gary S. Johnson \\
\hline Dr. Bimal K. Ray \\
\hline Dr. Leslie A. Lyons \\
\hline Dr. Christine G. Elsik \\
\hline Dr. Robert D. Schnabel
\end{tabular}




\section{Acknowledgements}

First and foremost I would like to thank Dr. Gary S. Johnson for giving me the opportunity to work in his lab and guiding me through this PhD program. Dr. Johnson is an amazing mentor, not only for the free meals and frogs, but he made a permanent mark in my life with his kind nature, quirky and dry sense of humor, patience, hard work and perseverance. I would like to express my gratitude to my dissertation committee, Dr. Bimal K. Ray, Dr. Leslie Lyons, Dr. Christine G. Elsik, and Dr. Robert D. Schnabel for all the helpful comments and guidance throughout my program. Each of you has had a very positive impact on me. I am thankful to have learned so much from you and your different areas of expertise; those are useful skills that I will carry throughout my professional life.

I would also like to thank all the lab crew: Dr. Tendai Mutangadura, Dr. Juyuan Guo, Liz Hansen, Peggy Ann Eichen, Natalie Villani, and the so many other members that have come and gone throughout the years. I also acknowledge Dr. Dennis O'Brien, Dr. Martin Katz, Dr. Joan Coates, and the many other research collaborators. I am also extremely thankful for all the support and encouragement I received from all of my family. My family was always there for me and helped to motivate me through the hard times, even from far away. A special thank you to my lovely boyfriend Thomas Stauffer, and to my adoptive American family: Donna and Don Stauffer, and Brian and Alexandria Stauffer, who were always supportive and believed in me.

Last but not least, I would like to thank my friends in Columbia, Guilherme Hosotani, Mariana Masiero, Estela Kobashigawa, Francine Russo, Brock Duck, Tim Poindexter, Sidharth Sen, Barbara Gandolfi and Erica Creighton. And my friends who are far away and still being supportive, Nathaly Gasparin, Mieke Stevens, Stefan Norenius, Brian Davis and Fabiana Farias. 


\section{Table of Contents}

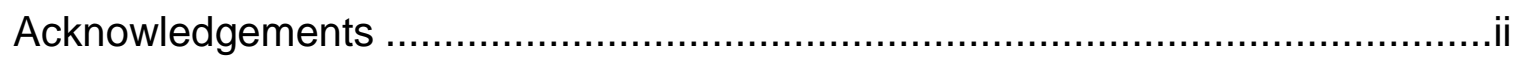

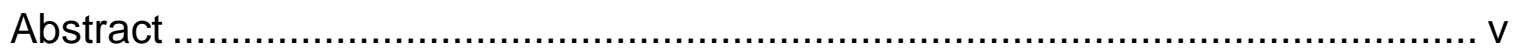

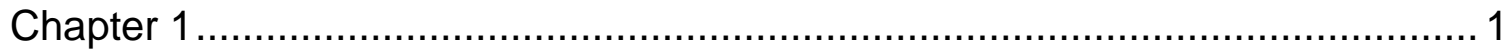

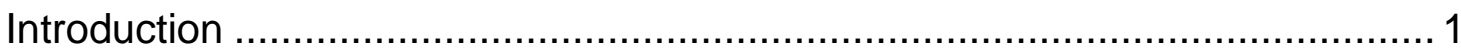

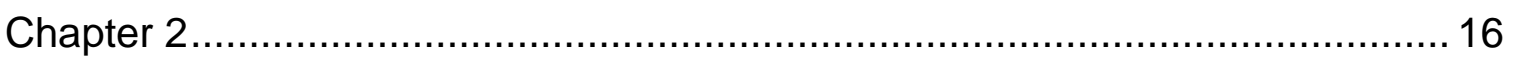

A homozygous PIGN missense mutation in Soft Coated Wheaten Terriers with a canine paroxysmal non-kinesigenic dyskinesia ............................... 16

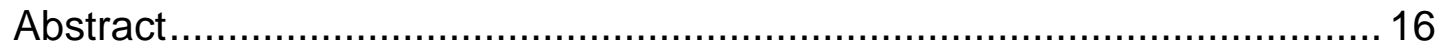

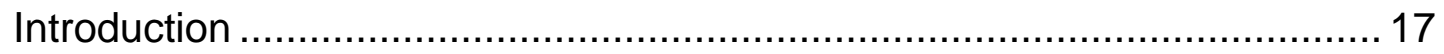

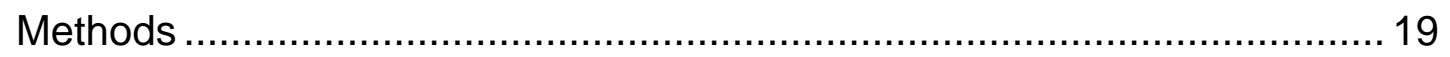

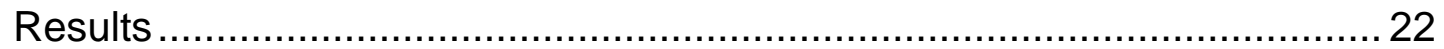

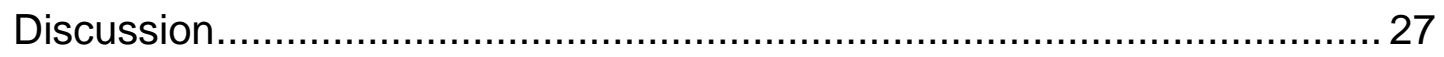

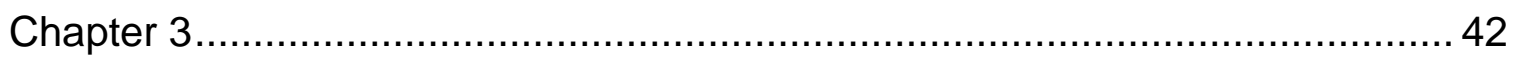

Australian Cattle Dogs with Neuronal Ceroid Lipofuscinosis are Homozygous for a CLN5 Nonsense mutation Previously Identified in Border Collies ........... 42

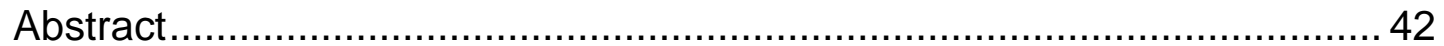

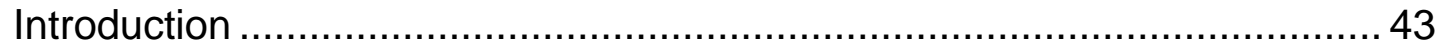

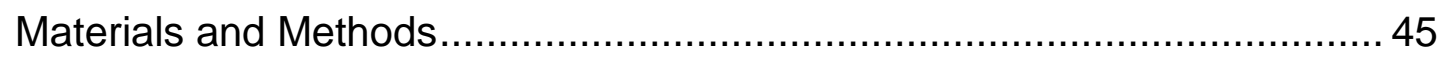

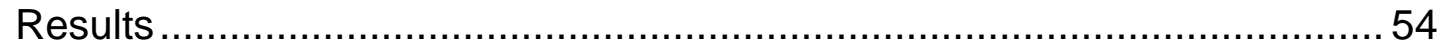

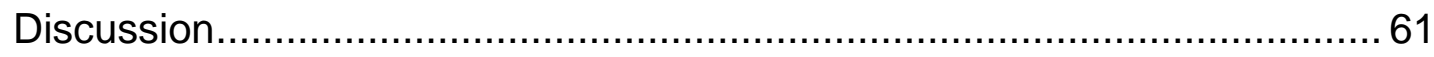

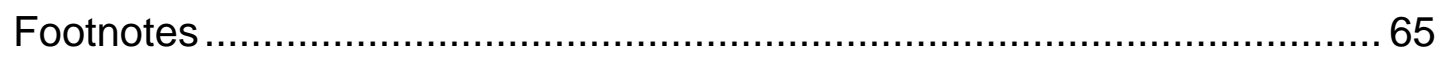

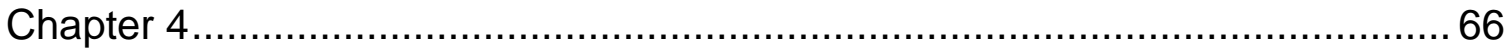

A homozygous PPT1 splice donor mutation in a Cane Corso Dog with neuronal

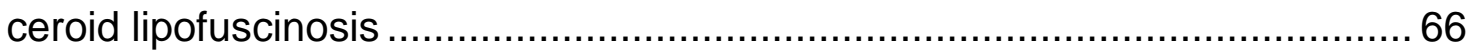

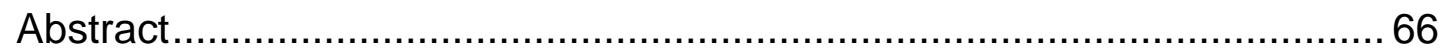

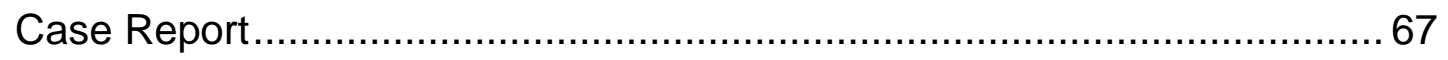

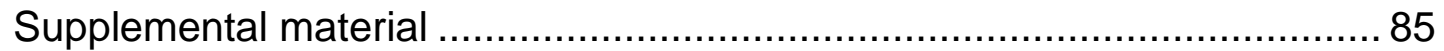

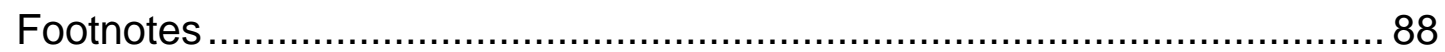

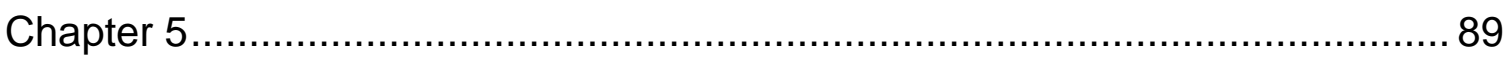


GM2 gangliosidosis in Shiba Inu dogs with an inframe deletion in HEXB .......89

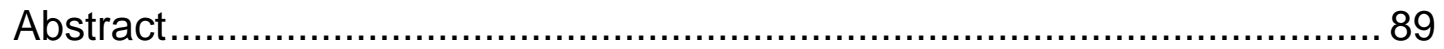

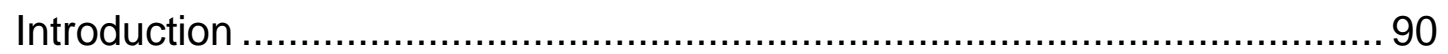

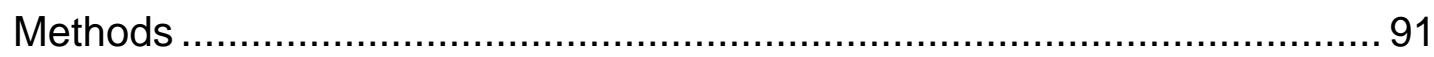

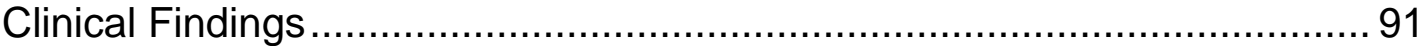

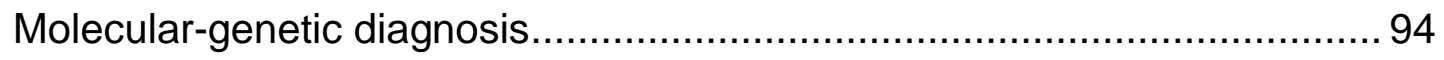

Biochemical and electron microscopic confirmation.................................... 95

Autofluorescent storage bodies in NCL and other diseases..................... 101

Comparison with other gangliosidoses in dogs ..................................... 103

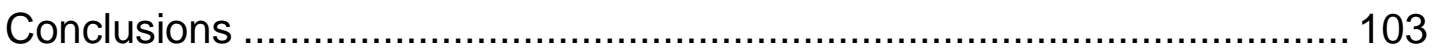

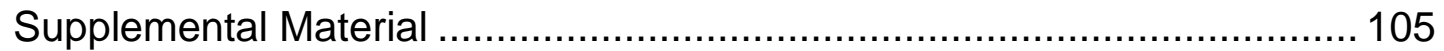

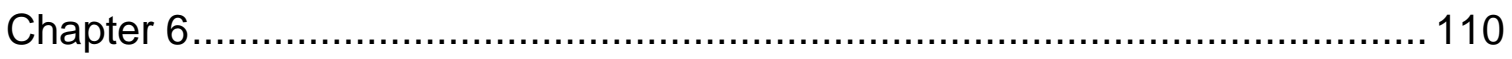

Discussion and Conclusion ............................................................... 110

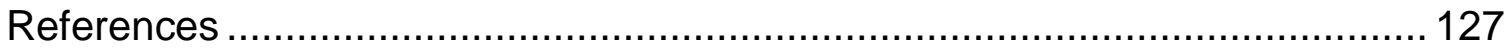

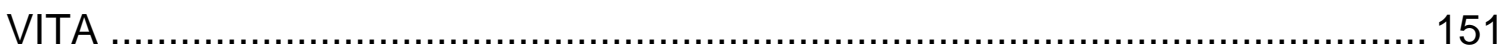




\section{Abstract}

This dissertation focuses on the use of whole genome sequencing (WGS) for the identification of disease causing variants in canine genomes. A brief review on the historical milestones of genetics, the creation and popularization of the fast throughput DNA sequencing technologies and their advantages and potential problems and biases, the importance of the study of canine genetics and the current state of the canine genome assembly is presented. Our lab sequenced $\sim 100$ dogs in the attempt to discover disease-causing variants. So far 20 such variants have been identified. This dissertation contains detailed accounts of the discovery variants likely to be responsible for four canine diseases. Those diseases are: Paroxysmal dyskinesia in Soft Coated Wheaten Terriers that is associated to the missense mutation PIGN:C.398C $>\mathrm{T}$; two different forms of neuronal ceroid lipofuscinosis, one in Australian Cattle dogs caused by CLN5:C.619C>T, and one in the Cane Corso caused by the splice site mutation PPT1C.124+1G>A; and a Shiba Inu GM2 gangliosidosis caused by HEXB.c:948_950deICCT. Furthermore, examples of not so successful attempts, possible reasons for failures and suggestions to successfully conclude other ongoing investigations. 


\section{Chapter 1}

\section{Introduction}

Next-generation sequencing (NGS) is one of the current technologies used for genetic variation screening and novel variant discovery for personalized medicine. Due to decreased costs and fast turnaround, NGS is also becoming viable option for personalized veterinary medicine. This dissertation focuses on the use of whole genome sequencing (WGS) for the identification of disease-causing variants in canine genomes. This introductory section reviews important milestones in the history of genetics and sequencing technologies. In addition it describes how the newer technologies have contributed to the establishment of personalized medicine and how WGS is contributing to the study of canine genetics and inherited canine diseases.

Genetics as a science and formal discipline is relatively young historically. Mankind, however, has been wondering about the origins of life, and how some traits are transmitted through generations since the Greek ancient times. For example, Herodotus produced one of the first known descriptions of a genetic condition in humans in one of his book passages: "The goddess [Aphrodite] afflicted the Scythians... and all their descendants forever with hermaphroditism..."1. Then attributed to Gods, curses, and the supernatural, several familial diseases, such as epilepsy, dwarfism, hemophilia, color blindness,

polydactyly and albinism, were anecdotally described by ancient physicians. ${ }^{1}$ The 
scientific community formally started to document their philosophies and theories regarding genetics in the early 1800 s, more specifically when Jean-Baptiste Lamarck wrote the first coherent and occasionally still discussed theory of evolution. ${ }^{2,3}$ In 1858 Charles R. Darwin published the widely accepted concepts of natural selection and evolution. ${ }^{4}$ Simultaneously, Gregor Mendel described "Mendelian inheritance" based on his observations while breeding pea plants. ${ }^{5,6}$ In 1871 Friedrich Miescher was the first scientist to isolate DNA from the nuclei of white blood cells, which he then called "nuclein." was unrecognized until much later, when in 1944 the link between the DNA molecule and heredity was established by Avery, MacLeod and McCarty. 6,9 Scientists world-wide became excited with the discovery and the "DNA era" began. The structure of DNA was described by James Watson and Francis Crick in the 1950s. ${ }^{6,10}$ Their discovery was based on the X-ray crystallographic investigations by Rosalind Franklin and Maurice Wilkins, ${ }^{10,11}$ together with the previous knowledge generated through biochemical studies of nucleotides by Phoebus Levene $^{10,12}$ and Erwin Chargaff. ${ }^{10,13,14}$ Twelve years later, scientists could sequence nucleotides through fragmenting RNA into short oligonucleotides with various RNase enzymes and then identify the residues using two-dimensional chromatography and spectrophotometry. With this extremely laborious methodology, only a few base pairs per year could be sequenced, not nearly enough to sequence an entire gene..$^{15}$ In 1977, Sanger and Maxam and Gilbert developed novel sequencing methods. ${ }^{16,17}$ Sanger's method prevailed over the Maxam and Gilbert's method because it is simpler, more reliable and used fewer 
toxic chemicals. ${ }^{18}$ Polymerase chain reaction (PCR) was invented by Kary Mullis in 1983, when he was working for Cetus Corp as a chemist. Mullis won the Nobel prize for inventing PCR in $1993 .{ }^{19}$ The invention of PCR technology allowed the automation of Sanger sequencing and revolutionized the genetics field, and the establishment of GenBank (http://www.ncbi.nlm.nih.gov/genbank) resulted in the generation of thousands of sequences through the 1980s, 1990s and early 2000s. ${ }^{18,20-22}$ Automated Sanger sequencing is still widely used for sequencing short segments of DNA and confirming genotypes from other technologies, among other applications..$^{20,21,23}$ Many different fields currently benefit from the different methodologies of nucleotide sequencing and genotyping including: forensics sciences, evolution research, medical research, cloning, genetically modified organisms, gene therapy, animal breeding, crop improvement, and environment conservation genetics, among others.

The first complete human whole genome sequence was done by the International Human Genome Sequencing Consortium, funded by the National Human Genome Research Institute (NHGRI). The human genome project (HGP) took 13 years for completion (1990-2003) and had a cost of over 2.7 billion dollars. ${ }^{24} \mathrm{~A}$ second human genome sequence was released nearly simultaneously by the Celera Corporation, led by Craig Venter and colleagues. ${ }^{25}$ Those sequences were generated with slightly different "shotgun sequencing" methods. Shotgun sequencing consists in random sequencing of sheared genome fragments, for a later contig construction. The HGP opted for a "hierarchical shotgun sequencing" approach that involved the generation and organization of a set of large-insert 
bacterial artificial chromosomes (BACs) clones (100-200kb) that cover the genome separately and in an ordered manner, followed by the shotgun sequencing and assembly of the BAC-clones hierarchically, as an attempt to diminish the risk of misassembly. ${ }^{24}$ Celera's group used a mixed strategy, involving some coverage with whole-genome shotgun data together with the publicly available hierarchical shotgun data generated by the International Human Genome Sequencing Consortium. ${ }^{25}$ HGP's assembly process involved filtering, to uniformly eliminate contamination from nonhuman sequences and other artifacts, placing the sequences on the physical map, and merging the sequences generating sequence contigs. ${ }^{24}$ The merged BAC sequences had fairly large contigs (half with over 80 $\mathrm{kb}$ ) and represented $\sim 90 \%$ of the euchromatic portion of the human genome, with a total sequence coverage of 7.5 -fold. Celera's assembly strategy was a "joint assembly" of Celera's WGS data (5.1-fold coverage) and HPG's data. The Celera's joint assembly output did not contain any dramatic differences other than adding a few percent additional sequence and ordering information, however, their approach demonstrated that it is possible to use whole-genome shotgun sequencing for assembling large genomes, which is much faster, easier and costeffective than the hierarchical shotgun sequencing methodology. ${ }^{26}$

High throughput massively parallel sequencing technologies started to appear around 2005. These technologies substantially reduced sequencing times and costs. Since then, the world has been experiencing a booming "genomic era." 18 The impressive advances of the "genomics era" can be attributed to the concomitant rapid technological advances in the fields of computer science, 
computer engineering, biochemistry and bioengineering. Nowadays, a human genome can be sequenced with $\sim 30 \mathrm{X}$ average coverage in just a few days, for a total cost of $\sim \$ 2000$ with the newest DNA sequencing technologies, and in the future a genome may possibly be sequenced for about $\$ 1,000$ or less in about 15 minutes. ${ }^{20}$ The current challenges involve data storage, interpretation and integration, which are further discussed in this chapter.

Due to the increased sequencing reaction speed, cost reduction and technical ease, the amount of genomic data is rapidly increasing and has become popular in interspecies research. Currently, genomes from 277 different vertebrate species have already been sequenced and there is an ongoing "Genome 10k project" that aims to assemble a collection of DNA sequences representing the genomes of $\sim 10,000$ vertebrate species. ${ }^{27,28}$ There is an estimate is that by the year 2025, researchers will be producing between 2 and 40 Exabyte of genomic data per year. ${ }^{29}$ The more good quality data available, the more accurate the cumulative databases will become, which will facilitate data interpretation in the future.

The dog is among the many species with a sequenced reference genome, and is a very important biomedical animal model. ${ }^{30}$ Because dogs mostly share the same environment as humans and have a relatively similar physiology, the study of the dog genome can provide valuable information about our own evolutionary and immigration history and health. ${ }^{31}$ In addition, these studies may lead to the discovery of novel genetic pathways that contribute to disease and other phenotypes, such as body size and skull shape. ${ }^{32}$ Dog genome studies can also 
lead to the development of DNA tests that are powerful diagnostic tools, which can help breeders eliminate undesired traits..$^{30,33}$

Many details about the origin of the domestic dog origin are still under debate, such as place of origin (Europe or Asia) and the number of domestication events. ${ }^{34}$ The consensus among experts is that dog domestication occurred sometime between $\sim 15,000-100,000$ years ago and involved several levels of admixture between different domestic dogs and wolves. ${ }^{31,35-38}$ Breed formation took place more recently and currently $\sim 350$ registered dog breeds exist. Not surprisingly, genetic evolutionary and population studies revealed that the canine species has a very unique population structure and an extreme genome plasticity, ${ }^{39}$ which is explained by the two major genetic bottlenecks, domestication and breed formation, that are responsible for shaping the current canine haplotype structure and linkage disequilibrium (LD). Intra-breed LD in dogs can extend for several Mbs, meaning individuals of the same breed are relatively genetically homogenous, whereas the interbreed LD extends for only a few kbs. The canine haplotype and LD structure bring advantages for genome-wide association studies (GWAS), as regions associated to certain traits (mostly Mendelian) can be detected using fewer markers than required for other species, such as humans, which have shorter LD ${ }^{39}$

The first canine whole genome assembly was generated in 2003 through wholegenome shotgun sequencing of a male standard poodle, with an average of $1.5 \mathrm{X}$ coverage (6.22 million reads), which covered $77 \%$ of the coding genome of a sequence of four overlapping BACs that provided a $512 \mathrm{~kb}$ reference of the dog genome, with 265 gaps of 452 bases mean length. ${ }^{40}$ In 2005, a female boxer with 
a high degree of homozygosity was selected and sequenced for an improved version of the canine genome reference sequence by the Broad Institute. ${ }^{41} \mathrm{~A}$ whole-genome shotgun sequencing approach was used to produce a reference sequence that spanned a total of 31.5 million reads providing $\sim 7.5 \mathrm{X}$ coverage. The first assembly (CanFam 1.0) was generated using an updated version of the ARACHNE program. ${ }^{42}$ An updated assembly (CanFam 2.0) had minor improvements, such as longer contigs and fewer genic gaps. The sequence contigs of the boxer sequence were $\sim 50$-fold larger than the standard poodle sequence, and $97 \%$ of the assembled sequence was anchored to the canine chromosomes. However even with the improvements and high quality, 1\% of the euchromatic genome resided in gaps and $\sim 20 \%$ of genes show some evidence of sequencing errors, such as frameshifts, very short introns, and missing first exons and promoters. The most current build (CanFam3.1) improved the quality and completeness of the canine genome sequencing by closing the small gaps with 12,101 primer walks, and closing the larger gaps and correcting mis-assembled regions through the re-sequencing of targeted BACs. In total, $85 \mathrm{Mb}$ of novel sequence was integrated into CanFam2.0 to create CanFam3.1. The new assembly (CanFam3.1) covers $99.8 \%$ of the euchromatic portion of the genome, and it also provides a much improved annotation due to RNA-sequencing data sets from 10 different canine tissues. ${ }^{43}$

The early canine gene mapping studies were done by linkage analysis with sparse numbers of microsatellite markers. ${ }^{44}$ With the availability of the high quality draft of the canine reference sequence, microsatellite technology was rapidly replaced by 
high density SNP arrays. ${ }^{30}$ Linkage mapping and GWAS research have been effective in identifying the chromosomal locations of genes responsible for many Mendelian traits, including diseases. One of the first successes for identification of a disease-linked marker occurred in 1997 with the detection of a microsatellite linked to the canine copper toxicosis in Bedlington Terriers. ${ }^{45}$ Other successful microsatellite studies identified the first canine epilepsy gene (a canid-specific unstable dodecamer repeat in $E P M 2 B)^{46}$ and the cause of dwarfism in German Shepherds (deletion of one of six 7 bp repeats in intron 5 of $L H X 3$ ).$^{47}$ GWAS studies using high density SNPchips and the improvement of the reference sequence increased the resolution and sensitivity of the mapping and also allowed researchers to use unrelated animals in their experimental design. Well known successes with this technology include identification of the cause of the hair ridge in Rhodesian ridgebacks, ${ }^{48}$ the finding that hair coat variation that is determined mostly by 3 genes (RSPO2, FGF5, and KRT71) 49 and the discovery of a mutation in $S O D 1$ that is a risk factor to degenerative myelopathy in many breeds, ${ }^{50}$ among many other important studies.

While the use of high density chips for GWAS mapping is still relevant for disease locus discovery, whole genome sequencing via NGS is becoming the preferred technology. In some instances a whole genome sequence from a single affected individual has led to the identification of causal variants because previously generated sequences from individuals from different breeds and different disease phenotypes have been used as controls..$^{51-55}$ 
Next-generation sequencing refers to high throughput sequencing technologies and includes multiple sequencing platforms, e.g. Illumina, Inc, ${ }^{56}$ Life Technologies Corp, ${ }^{57}$ Pacific Biosciences, Inc. ${ }^{58}$ among others. The Illumina platform is the most commonly used for canine genetics research due to cost benefits. Typically, the average read length of the Illumina platform is $\sim 100$ bp per read pair with an average $\sim 30 \mathrm{X}$ coverage along the genome, which is enough to identify a good portion, but unfortunately not all, genome variation. The shorter read lengths may not align to a reference genome uniquely in repetitive regions, so interpreting copy number variation and large deletions and insertions can be challenging. Longer read lengths are desirable for detecting such large genome variations, however the current long read technologies are not yet cost effective for mass genotyping, and their use is so far limited to de novo assemblies. ${ }^{59}$

While sequence data generation has become routine, data analysis and interpretation remain challenging. The creation of databases and pipelines are essential for quality research. In general, the steps for NGS data analysis include data tracking, barcoding, storage, quality control, sequence alignment to the reference genome, variant calling and interpretation. Data tracking pertains to good practices in maintaining databases and linking genotype data to a certain individual and phenotype information. Barcoding assures that the correct output is attributed to the correct sample when multiplexing different samples and libraries. Barcodes are unique DNA sequences of approximately 8-12 bp that are ligated to fragments within the sequencing library, and can be identified and sorted using bioinformatics software. ${ }^{60}$ 
Data storage is an important concern because genomes are large, therefore the sequencing files are also large. For example, a canine WGS with an average of $30 \mathrm{X}$ coverage uncompressed FASTQ file has typically $\sim 100-180 \mathrm{~GB}$, which is why compressing FASTQ files for storage is helpful. Sequence Alignment Map (SAM) format is the aligned FASTQ file, and contains more information than the raw FASTQ, and its usually compressed into a Binary Alignment Map (BAM) format. This can reduce the file size to $\sim 30-50 \mathrm{~GB} .{ }^{61}$ The researcher needs to consider the raw data file sizes and add extra room for intermediate processing files in order to make the right choices when purchasing the hardware to store their data.

The researcher must be cautious to avoid possible biases that can be introduced in multiple steps of the data generation and analysis. Sample bias can be avoided by assuring that the sample contains undegraded DNA of sufficient concentration $(10-20 \mu \mathrm{g})$, is free of contaminants, and that the EDTA concentrations not higher than $1 \mathrm{mM} .{ }^{62}$ The method of library preparation can be either PCR-based or PCRfree. The PCR-based methods tend to introduce PCR amplification bias (over representing the more efficiently amplified sequences) with lower coverage in high GC\% and repetitive content regions. ${ }^{63}$ Mispriming events may also occur in PCRbased libraries, which can then introduce false positive variation in certain regions of the genome, especially in samples with low DNA quantity or few sequencing reads. ${ }^{64}$ PCR-free libraries can eliminate most of the coverage biases introduced by PCR-based libraries. However, very high GC-rich areas (GC\% $\geq 75)$ can still be problematic. ${ }^{65}$ There are a few downsides to the use of PCR-free libraries that can be easily overcome. For example, the library preparations require relatively large 
amounts of sample DNA, that might not be feasible for some sample types, and the method is more sensitive to variations in DNA quality and purity. Because the PCR-free protocols are harder to automate, high-throughput sequencing facilities do not opt PCR-free as the default protocol.63 The library quality control (QC) consists of verifying if the insert sizes are as expected and if there are contaminating adapter-dimers.

In addition to coverage bias, different NGS technologies can introduce chemistry error biases, especially in regions adjacent to long homopolymers, GC extremes, deletions and insertions. ${ }^{65}$ Because of the possible base calling errors, Phred quality scores are generated for each sequenced base. Phred quality scores or $Q$ are logarithmically related to base-calling error probabilities and should be generally above Q30 (chances that the base is called incorrectly is 1 in 1000). Reads with low overall $Q$ values are usually discarded from the analysis. ${ }^{66}$ There are many different choices for quality control (QC) software and metrics that can be used to guarantee the data is useful during multiple steps of the pipeline (raw data, aligned data and variant calling). The researcher should evaluate which one is the most relevant for their specific type of data. ${ }^{67}$ Further details about some of those different methods are discussed in chapter 6 as personal experiences.

Even though there are still issues, NGS is becoming the gold-standard for clinical cytogenetics and personalized medicine. ${ }^{68}$ For example, whole exome sequencing has been used in human medicine as an alternative method for cost-effective clinical research, e.g. the genetic diagnose for Bartter syndrome, ${ }^{69}$ autoimmune lymphoproliferative syndrome (ALPS) ${ }^{70}$ and childhood inflammatory bowel 
disease..$^{71}$ Recent association studies found that $>80 \%$ of disease-associated sequence variants fall outside of coding regions ${ }^{72,73}$ and targeted exome sequencing can miss important regulatory variation. Therefore, whole genome sequencing (WGS) is now more cost effective and is becoming routine in the clinical practice and personalized medicine ${ }^{74,75}$ WGS can be successfully used to alter the treatment plan of a patient with cancer $^{76}$ and identify novel diseaserelevant preclinical risk factors for complex diseases such as cancer. ${ }^{77}$ It can also be used as a non-invasive method to detect organ transplant rejection ${ }^{78}$ and detect fetal Down syndrome and other fetal aneuploidies. ${ }^{79}$

The benefits of WGS for the identification of disease causing variants can also be translated to veterinary medicine, and several canine genome researchers are using this technology. Chapters 2 to 5 describe examples of 4 successes achieved with this effective methodology, in the order of their discovery. Chapters 2 through 4 are copies of the reports as published in peer-reviewed journals. The Manuscript in chapter 5 has been accepted after peer review and modification by the copy editor. This draft has received final editorial acceptance. Chapter 6 is a discussion of the overall research efforts during my graduate studies at the Univerity of Missouri and concluding remarks about these research efforts.

Chapter 2 has been published in Neurogenetics (https://www.ncbi.nlm.nih.gov/pmc/articles/PMC5243907/pdf/10048 2016 Article 502.pdf). ${ }^{80}$ This chapter describes a paroxysmal dyskinesia in Soft Coated Wheaten Terriers caused by a nonsynonymous variant in PIGN. For this publication, my role was to validate the candidate allele PIGN:C.398C >T using a 
PCR-restriction fragment length polymorphism (PCR-RFLP) assay and then to genotype $\sim 1200$ animals using an allelic discrimination assay. ${ }^{81} \mathrm{I}$ also assisted with the writing of the molecular genetics part of the Materials and Methods and generated the Supplemental Tables and Figure.

Chapter 3 has been published in the Journal of Veterinary Internal Medicine (https://www.ncbi.nlm.nih.gov/pmc/articles/PMC5084771/pdf/JVIM-30-

1149.pdf). ${ }^{82}$ This article describes the clinical histories of four Australian Cattle Dogs with neuronal ceroid lipofuscinosis (NCL). All four dogs were homozygous for a nonsense mutation in CLN5, which had been previously identified as a cause of NCL in Border Collies. This was the first report that the same variant allele was responsible for NCL in more than one breed. For this study, I processed the WGS data for the affected dog and 43 control dogs through the data analysis pipeline. I also aligned the error corrected reads to the canine reference sequence and generated the variant reports. In addition, I sequenced the four affected Australian Cattle Dogs by direct Sanger sequencing of a variant-containing PCR amplicon and used a TaqMan allelic discrimination assay to genotype over 700 dogs for the variant allele. In addition, and I wrote the first draft of the Materials and Methods and generated Table 2 .

Chapter 4 is published in the Journal of Veterinary Internal Medicine (https://www.ncbi.nIm.nih.gov/pmc/articles/PMC5259623/pdf/JVIM-31-149.pdf). ${ }^{53}$

This chapter contains a case report of a NCL in a single female Cane Corso dog. The diagnosis was based on clinical signs and results from light, fluorescence and electron microscopy. A splice donor mutation in a known NCL gene 
$(P P T 1 C .124+1 G>A)$ was identified as the probable disease-causing variant by whole genome sequencing. For this report, I identified the causal mutation in the whole genome sequence by aligning the affected dog sequence reads to the canine reference sequence and visually inspecting the sequences of the 13 known NCL-causing genes using a genome browser (IGV). I used an online tool (http://genes.mit.edu/burgelab/maxent/Xmaxentscan scoreseq.html) to estimate the splice donor strength of the nucleotide sequences surrounding the canonical splice site. I also validated the mutation using Sanger sequencing of a variantcontaining PCR amplicon. In addition, I designed a nested primer, two-stage allelic discrimination assay to genotype for the causal splice donor variant which was located in a particularly difficult chromosomal region due to the highly repetitive and GC-rich content. I used this two-stage allelic discrimination assay to genotype a cohort of Cane Corso samples. I wrote the first draft of the Materials and Methods section, the Supplemental material and table, and generated the tables in the text and Figure 6.

Chapter 5 is the text of a peer reviewed and accepted article for the Journal of Veterinary Internal Medicine. This case report describes two Shiba Inu Dogs with clinical signs and autofluorescence consistent with NCL. The individual cases were referred to us from independent sources. Based on clinical signs and the finding of autofluorescent storage bodies in affected-dog neurons, the initial diagnosis was NCL. However, WGS failed to identify a candidate variant in a known NCLassociated gene. Instead, a 3 bp inframe deletion in HEXB was identified in a whole genome sequence from one of the affected Shiba Inu, suggesting a 
probable diagnosis of GM2 gangliosidosis. This diagnosis was confirmed by thin layer chromatography and enzymology. For this report I generated and analyzed the variant reports for the affected dog and other 105 whole genome sequences used as controls, identified the HEXB.c:948_950de/CCT variation, and confirmed the 3 base pair deletion through PCR-RFLP. In addition, I wrote the first draft of the paper and generated the supplemental table and figure.

Chapter 6 is the discussion and conclusion segment of this dissertation. In this chapter, different methods, pros, cons, future prospects and challenges of WGS analysis and its applications in personalized veterinary medicine is further discussed. My personal experiences with the data analysis and interpretation, and hypotheses on ways to increase the success of causal variant discovery rate are also discussed. 


\section{Chapter 2}

\section{A homozygous PIGN missense mutation in Soft Coated Wheaten Terriers with a canine paroxysmal non-kinesigenic dyskinesia Abstract}

Hereditary paroxysmal dyskinesias $(\mathrm{PxD})$ are a heterogeneous group of movement disorders classified by frequency, duration, and triggers of the episodes. A young-adult onset canine PxD has segregated as an autosomal recessive trait in Soft-Coated Wheaten Terriers. The medical records and videos of episodes from 25 affected dogs were reviewed. The episodes of hyperkinesia and dystonia lasted from several minutes to several hours and could occur as often as $>10 /$ day. They were not associated with strenuous exercise or fasting but were sometimes triggered by excitement. The canine PxD phenotype most closely resembled paroxysmal non-kinesigenic dyskinesia (PNKD) of humans. Whole genome sequences were generated with DNA from 2 affected dogs and analyzed in comparison to 100 control canid whole genome sequences. The two whole genome sequences from dogs with PxD had a rare homozygous PIGN:C.398C>T transition, which predicted the substitution of an isoleucine for a highly conserved threonine in the encoded enzyme. All 25 PxD-affected dogs were PIGN:c.398T allele homozygotes, whereas there were no c.398T homozygotes among 1185 genotyped dogs without known histories of PxD. PIGN encodes an enzyme involved in the biosynthesis of glycosylphosphatidylinositol (GPI), which anchors a variety of proteins including CD59 to the cell surface. Flow cytometry of PIGNknockout HEK239 cells expressing recombinant human PIGN with the c.398T 
variant showed reduced CD59 expression. Mutations in human PIGN have been associated with multiple congenital anomalies-hypotonia-seizures syndrome-1 (MCAHS1). Movement disorders can be a part of MCAHS1, but this is the first PxD associated with altered GPI anchor function.

\section{Introduction}

The human paroxysmal dyskinesias (PxD) are a heterogeneous group of diseases characterized by episodes of abnormal involuntary movements. ${ }^{83}$ The episodes may last $<1$ min or continue for many hours. Episode frequency may vary from $<1 /$ year to $100 /$ day. The abnormal movements can be dystonia, chorea, athetosis, or ballism, either singly or in various combinations. ${ }^{84-86}$ Many PxD episodes are initiated by a recognized trigger, and the type of trigger has served as a means of subclassifying PxD. ${ }^{83,84,87}$ In paroxysmal exertional dyskinesia (PED), the episodes are triggered by sustained exercise. Sudden movements trigger the episodes in paroxysmal kinesigenic dyskinesia (PKD). In paroxysmal non-kinesigenic dyskinesia (PNKD), stress, fatigue, and hunger can trigger episodes in some patients, but the most consistent triggers are alcohol or caffeine consumption. Other factors considered in classification are age of onset, frequency and duration of the episodes, and response to therapy. ${ }^{83,84,87-89}$

PxD may be secondary to a specific etiology such as encephalitis or stroke ${ }^{90}$ or idiopathic. The latter can be sporadic or familial, usually with an autosomal dominant inheritance. ${ }^{87}$ Mutations in three genes, SLC2A1, PRRT2, and PNKD (also referred to as MR-1), are well-recognized causes of PxD. ${ }^{87,91-95}$ SLC2A1 
encodes glucose transport protein 1 (GLUT1), which facilitates glucose transfer across the blood-brain barrier. Most patients with heterozygous SLC2A1 mutations exhibit GLUT1-deficiency syndrome characterized by low CSF-to-blood glucose ratios together with epilepsy, developmental delay, microcephaly, ataxia, and PxD. ${ }^{96}$ A minority of patients with heterozygous SLC2A1 mutations develop isolated PED. ${ }^{93,97-99}$ PRRT2 encodes proline-rich transmembrane protein 2, which interacts with SNAP25 and thereby influences the release of glutamate or other neurotransmitters. ${ }^{100}$ Heterozygous PRRT2 mutations have been found in patients with several paroxysmal neurologic disorders including PKD. ${ }^{101}$ Many PNKD patients carry heterozygous mutations in PNKD. $87,88,95$ This gene encodes a protein that may suppress synaptic vesicle release by interacting with RIM1 and RIM2. ${ }^{102}$ Although SLC2A1, PNKD, and PRRT2 harbor the majority of the causal mutations for the genetically defined cases of $\operatorname{PxD},{ }^{87}$ a few patients have been reported to carry potentially causal mutations in other genes such as KCNMA1103 and PDHA1. ${ }^{104}$ Efforts to identify the mutations responsible for PxD in other families have not yet been successful. ${ }^{88}$

Dogs can also develop PxD. ${ }^{105}$ Published reports have provided clinical descriptions of primary canine PxD ( $C P x D)$ or $c P x D$-like diseases in several breeds including the Bichon Frise, ${ }^{106}$ Border Terrier, ${ }^{107}$ Cavalier King Charles Spaniel, ${ }^{108}$ Chinook, ${ }^{109}$ Doberman Pinscher, ${ }^{110}$ English Bulldog, ${ }^{111}$ Scottish Terrier, ${ }^{112}$ and Soft-Coated Wheaten Terrier (SCWT). ${ }^{113}$ Unlike the human primary PxD that are almost always dominant traits, the cPxD usually have a recessive mode of inheritance. The $\mathrm{cPxD}$ that occurs in the Cavalier King Charles Spaniel is typically 
precipitated by exercise. ${ }^{108}$ It is caused by a homozygous $16-\mathrm{kb}$ microdeletion that includes the first three exons of $B C A N^{114,115}$, which encodes the extracellularmatrix protein brevican. The molecular genetic causes of the other $\mathrm{CPxD}$ have not yet been reported. We now present evidence that SCWT with CPxD have a deficiency in the biosynthesis of glycosylphosphatidyinositol (GPI) anchors due to a homozygous missense mutation in PIGN, the gene that encodes a GPI synthesis enzyme, GPI ethanolamine phosphate transferase-1.

\section{Methods}

\section{Animals}

Medical records and available videos of dyskinesia episodes were reviewed for 22 SCWT and 3 "Whoodles" (SCWT × Poodle crosses) with CPXD. The median age of onset of signs was 2.25 years. Males and females were affected equally. All dogs had normal neurologic exams between the episodes. Episode duration ranged from several minutes up to $>4 \mathrm{~h}$, and the frequency of episodes ranged from 1 every few days to >10/day. In six cases, stress, excitement, or loud noises were thought to precipitate some attacks, but a clear trigger was not apparent in most instances. No dogs had a history of ingestion of alcohol or caffeine. Typical episodes consisted of rapid flexion and extension of the hind limbs with varying degrees of truncal dystonia (Supplementary Video: https://staticcontent.springer.com/esm/art\%3A10.1007\%2Fs10048-016-0502-

4/MediaObjects/10048 2016502 MOESM3 ESM.wmv). Most frequently, the flexion alternated irregularly between limbs, but sometimes both hind limbs were 
off the ground simultaneously (Fig. 1). In severe episodes, the front limbs were also affected. No consistent improvement in episodes was reported with antiepileptic drugs or benzodiazepines. The signs typically worsened with age, and six dogs were euthanized within 2 years after onset of signs due to the increasing severity of the episodes. MRIs were obtained from five affected dogs, and postmortem exams were performed on four dogs, but no abnormalities were identified in any of the examined brains.

\section{Molecular Genetics}

DNA was collected from 25 cPxD-affected dogs. In addition, we used archived DNA samples from 665 cPxD-free SCWT, 388 Poodles, and 132 randomly selected dogs from other breeds to validate the identified candidate causal variant. Whole genome sequences were generated with DNA from two different cPxDaffected SCWT. PCR-based DNA libraries were used for the first whole genome sequence as previously described. ${ }^{116}$ PCR-free DNA libraries and slightly modified procedures $^{117}$ were used for the second. Both data sets were deposited in the Sequence Read Archives (accession numbers SRX863100 and SRX863101). Previously described procedures were used for trimming adaptor sequences from the sequence reads, eliminating duplicate reads, error correction, and alignment to the CanFam3.1 canine reference genome sequence. ${ }^{52,116}$ Sequence variant information was uploaded to a custom PostgreSQL database, which also contained sequence variant information from the aligned whole genome sequences of normal canids or dogs with diseases other than CPXD. These other sequences served as controls for the current analysis. The University of Missouri 
DNA Core Facility generated 43 of the control dog genome sequences; the rest were provided by individuals at other institutions as indicated in the "Acknowledgements".

PCR primers 5'-GCTATACTAATATTTCACCGTTC-3' and 5'AAAATATAGTAAGTAATACACAA-3' were used to amplify a segment of genomic DNA containing candidate sequence variant PIGN:c.398C $>$ T, identified from the whole genome sequences, so that the sequence variant could be verified by direct automated Sanger sequencing. An allelic discrimination assay ${ }^{81}$ was used to genotype DNA samples from individual dogs at this sequence variant. PCR primers for this assay were 5'-TGTGGGATTTGATTCTCTTATTAATG-3' and 5'TCAATGACTCTTACCTTTGGCAAACATA-3'. The competing probe sequences were 5'-VIC-CCAGCTCCATGTGTACC-MBG-3' (reference C allele) and 5'-FAMCCAGCTCCATATGTACC-MBG-3' (variant T allele).

PIGN-knockout HEK293 cells were generated and transfected as previously described ${ }^{118}$ with human wildtype or mutant (T133I, T133S, or T133V) PIGN complementary DNA (DNA) cloned into pME, a strong SRa promoter-driven expression vector, or pTK, a medium TK promoter driven expression vector. These PIGN constructs had an HA epitope tag at the $\mathrm{N}$-terminus. Transfection efficiencies were determined by the luciferase activity of cell lysates. After 3 days of incubation, restoration of the cell-surface expression of CD59 was evaluated by flow cytometry. ${ }^{118}$ Levels of expressed wild-type and mutant HA-tagged PIGN in pME vector-transfected cells were determined by western blotting using an anti-HA antibody. 


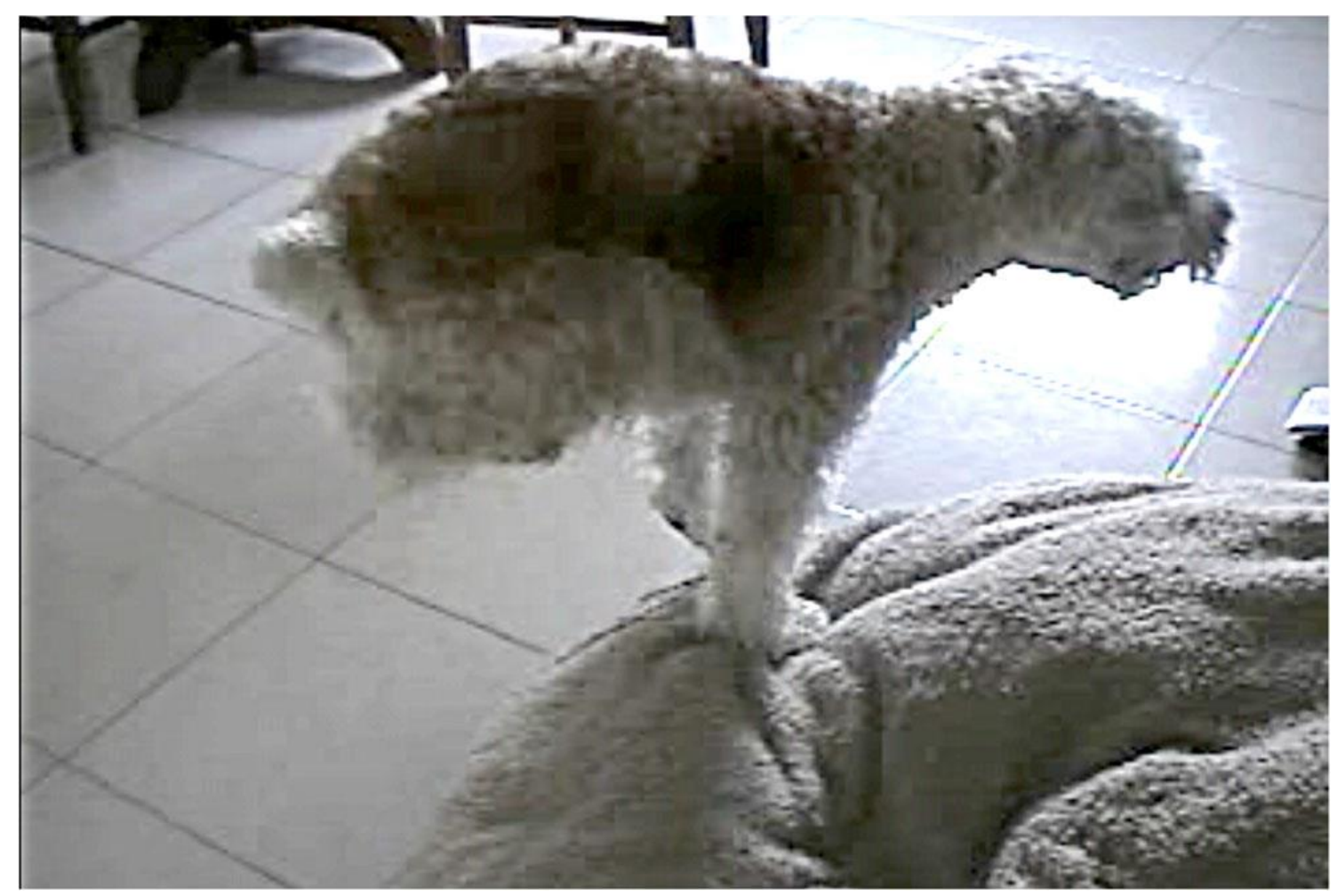

Fig. 1. A 4-year-old, SCWT during a dyskinesia episode showing hyperflexion of the hind limbs that elevated both limbs off the ground simultaneously.

\section{Results}

\section{Molecular genetics}

Partial pedigree information was available for some of the affected dogs. Because no records indicated that the sires or dams of any of the affected dogs also exhibited $\mathrm{cPxD}$, we assumed an autosomal recessive inheritance in our data analysis.

The first generated whole genome sequence from a cPxD-affected dog had a 21fold average coverage of the reference genome and contained 6.9 million potential sequence variants (homozygous or heterozygous differences from the reference canine genome sequence). When this sequence variant information was first 
added to a custom PostgreSQL database, it also contained sequence variant information from eight control canine WGSs. The SCWT sequence variants were filtered to identify only those that fit three criteria: (1) they were predicted to alter the amino acid sequence of the gene product (including those that alter exonsplicing signals), (2) they were homozygous in the cPxD-affected dog's whole genome sequence, and (3) they were absent from the control whole genome sequences. In the initial analysis, 418 sequence variants met these criteria. However, no plausible candidate sequence variants were identified from a review of the published biological functions and disease associations for the 302 genes that harbored these sequence variants.

As additional control whole genome sequences were added to the database, the number of homozygous coding variants unique to the affected SCWT decreased. Nonetheless, we were still unable to recognize a potential cPxD-causing sequence variant. Thus, we generated a whole genome sequence from another cPxDaffected dog. This sequence had 18 -fold average reference genome coverage and contained 5.5 million potential sequence variants. By the time that the variants from the second CPXD-affected SCWT were included in our database, it contained variant data for 100 canid sequences that could be considered to be controls for the SCWT PxD. At this time, the whole genome sequence for the first case SCWT contained 230 variants in 162 different genes that met our criteria for candidate causality (Supplementary Table 1a). The whole genome sequence for the second SCWT PxD case had 65 candidate variants in 54 different genes (Supplementary Table 1b). The higher number of variants from the first case is attributable to the 
many false positives among the filtered variants from the early PCR-amplified libraries. The only sequence variant common to both animals was PIGN:c.398C>T, which predicted a p.T133I substitution in PIGN, the gene product. Direct automated Sanger sequencing of PCR amplicons produced with primers spanning the PIGN:c.398C>T variant confirmed that both of the SCWT with CPxD were PIGN:c.398T homozygotes.

All 25 of our DNA samples from cPxD-affected dogs tested homozygous for the variant PIGN:c.398T allele. Twenty-two of these affected homozygotes were SCWT, and the other three were Whoodles. Whoodles are marketed as the product of F1 crosses between purebred SCWT and purebred Poodles. If this was indeed the origin of the three c.398T homozygous Whoodles, it would require that the PIGN:c.398T allele had been segregating in both breeds. Thus, we genotyped individual representatives from each breed at PIGN:C.398C>T. Fifteen of the 682 control SCWT were heterozygotes; the remaining 667 were homozygous for reference allele c.398C. All 388 of the genotyped Poodles were PIGN:c.398C homozygotes, as were all 132 randomly selected representatives of 92 other breeds. Although the PIGN:c.398T allele was not present in the Poodles that we genotyped, it could still be carried by other Poodles. Nonetheless, an alternative and more likely explanation for the origin of the three homozygous Whoodles is that they resulted from a Whoodle to SCWT backcross or from two Whoodle parents. 


\section{Flow cytometry}

The biological activities of mutant PIGN isoforms were assessed by transiently transfecting corresponding cDNAs into PIGN-knockout HEK293 cells and measuring the restoration of GPI-anchored CD59 surface expression. ${ }^{118,119}$ Flow cytometry of cells which were transfected with a medium promoter-driven expression vector revealed comparable restoration of CD59 cell-surface expression by PIGN-knockout HEK293 cells 3 days after transient transfection with wild-type human PIGN cDNA or with recombinant human cDNAs designed to encode PIGN:p.T133S or PIGN:p.T133V isoforms. However, transient transfection with recombinant human cDNAs designed to encode PIGN:p.T133I restored only $60-70 \%$ of the cell-surface CD59 that was produced by the wild-type cDNA (Fig. 2a). The same T133I PIGN cDNA, when expressed with a strong promoter-driven vector pME, nearly fully restored CD59 (Fig. 2a). Western blotting revealed comparably expressed protein levels of T133I and wild-type PIGN (Fig. 2b). It is therefore concluded that the $\mathrm{T} 133 \mathrm{I}$ mutation causes a reduction in specific activity rather than stability of PIGN. 
a
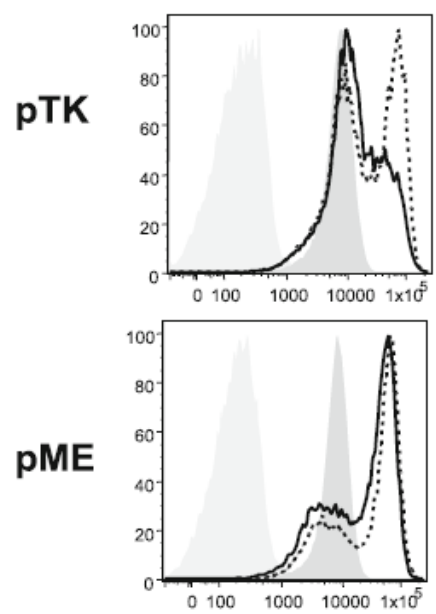

T133S
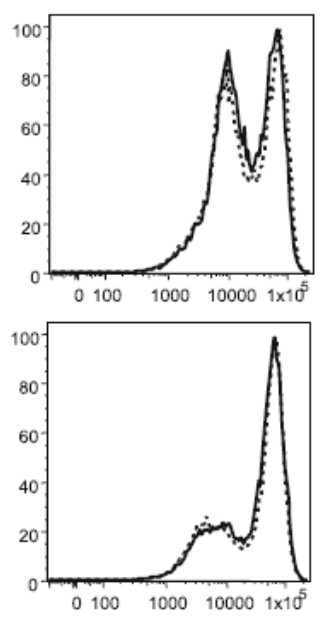

CD59
T133V
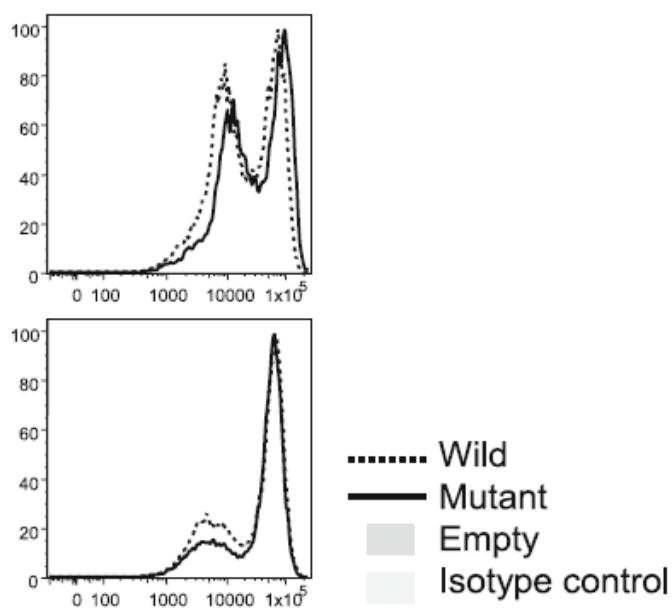

b

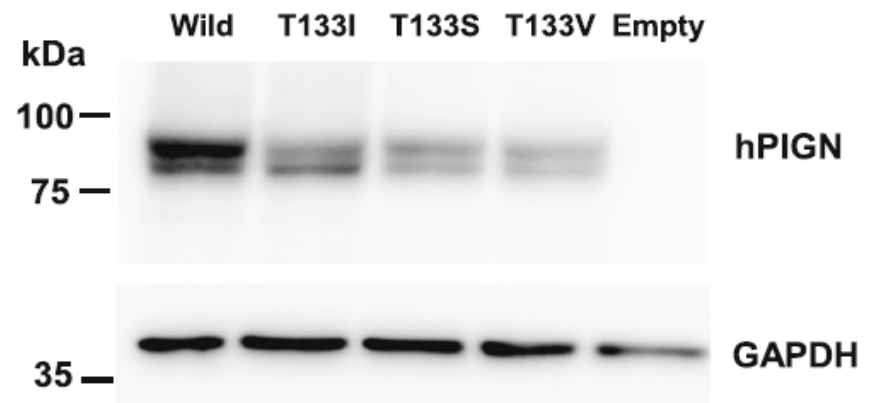

Fig. 2. Functional analysis of PIGN mutants in PIGN-knockout HEK293 cells. a. Restoration of cellsurface expression of GPI-anchored protein CD59 on PIGN knockout HEK293 cells after transfection. HA-epitope-tagged, wild-type T133I, T133S, and T133V human PIGN:cDNAs were transfected with a medium promoter-driven pTK vector (top) or a strong promoter-driven pME vector (bottom). Three days later, CD59 levels were assessed by flow cytometry. b. Western blotting analysis of wild-type T133I, T133S, and T133V human PIGN. PIGN-knockout HEK293 cells that were transfected with HA-epitope-tagged, wild-type and mutant PIGN cDNAs in pME were analyzed 3 days later by western blotting using anti-HA antibody. GAPDH glyceraldehyde 3phosphate dehydrogenase, a loading control. 


\section{Discussion}

We studied a cPxD originally described in SCWT in 2004, ${ }^{113}$ which shared features of human PxD. All the affected dogs were normal on examination between episodes. The movements appeared to be involuntary and could not be disrupted. The dogs remained conscious during the episodes, and except for severe episodes, the dogs continued to walk, suggesting that the movements were semipurposeful. Some of the movements in this CPXD were the sustained, abnormal postures of a dystonia. ${ }^{120}$ The more rapid movements in CPxD are hyperkinetic movements, but they are difficult to place into one of the human categories of chorea, ballism, or athetosis. ${ }^{121}$ The various forms of human PxD can be subclassified as a PED, PKD, or PNKD. ${ }^{83-86,88,89}$ The owners of dogs with $\mathrm{CPXD}$ never mentioned exercise or fasting as triggers of dyskinesia episodes as occurs with PED in humans. ${ }^{122}$ Determining whether a sudden movement triggered the episodes as in PKD in humans ${ }^{89}$ was difficult since the owners typically were not closely observing their dogs at the onset of the episodes. Compared to the durations and frequencies of episodes of dyskinesia in human PKD patients, ${ }^{89}$ the episodes in dogs with PIGN-associated cPxD lasted longer (minutes to hours) and occurred less frequently (several/day to every few weeks), more like PNKD in humans. ${ }^{88}$ Stress and excitement, which trigger episodes in most PNKD associated with $P N K D$ in humans ${ }^{88}$ were reported to trigger the episodes in some dogs, although in most cases, no trigger could be identified. While alcohol or caffeine ingestion are the most consistent triggers of human PNKD associated with PNKD mutations, ${ }^{88}$ dogs rarely ingest alcohol and methylxanthines are toxic to 
dogs, ${ }^{123}$ so whether they would trigger episodes in dogs is unknown. PKD patients either require no treatment or respond to anti-epileptic drugs, and PNKD patients often respond to benzodiazepines, ${ }^{84,87-89}$ while SCWT with $\mathrm{CPXD}$ showed a poor response to these drugs. The median age of onset in dogs was 2.25 years, a young but fully mature adult dog, which would be older than the equivalent average age of onset in humans of PNKD associated with PNKD or PKD and more comparable to "atypical" PNKD not associated with $P N K D$ mutations. ${ }^{88,89}$ Thus, even though there are differences, the dyskinesia episodes in PIGN-associated CPxD most closely resemble those of human PNKD patients.

To identify the molecular genetic cause of $\mathrm{cPxD}$, we generated whole genome sequences for two affected dogs. The T allele of a PIGN:c.398C >T transition was the only rare, homozygous, predicted amino acid altering sequence variant that was found in both of these animals. All 25 of our c.398T allele homozygous DNA samples were from cPxD-affected dogs, whereas none of the 1053 control SCWT and Poodles and none of the 132 genotyped dogs from other breeds were c.398T homozygotes. This established a strong association between the clinical disease and T allele homozygosity. None of the 15 PIGN:c.398C/T heterozygous SCWT were known to have exhibited episodes of dyskinesia, which supports the inheritance of SCWT CPxD as an autosomal recessive trait.

PIGN is 1 of more than 26 genes that encode the polypeptides which contribute to the biosynthesis, protein attachment, and remodeling of GPI anchors. GPI anchors tether a diverse group of cell-surface proteins to the plasma membrane (Fig. 3a). Amide bonds covalently attach the cell-surface protein to an ethanolamine 
phosphate at the outer end of the GPI anchor, while at the inner end, aliphatic chains intercalate into the plasma membranes and bind to hydrophobic lipid-raft components. A complex core glycolipid provides a covalent bridge between the two ends of the GPI anchor. ${ }^{119,124}$ During GPI assembly in the endoplasmic reticulum, ethanolamine phosphate side chains are added to mannosyl moieties in the core glycolipid. GPI ethanolamine phosphate transferase-1 (PIGN), the enzyme encoded by PIGN, facilitates the transfer of an ethanolamine phosphate from phosphatidylethanolamine to the innermost mannosyl moiety in a partially assembled core glycolipid intermediate (Fig. 3b). ${ }^{125-127}$ The existence of PIGNassociated deficiency diseases indicates that this ethanolamine phosphate side chain has an important biological function, probably related to its role in the recognition of GPI glycolipids by the transamidase complex that attaches proteins to the GPI anchors. ${ }^{128}$ Flow cytometry has been used to demonstrate altered expression of these proteins with mutations of PIGN in humans. ${ }^{118,129-131}$ 


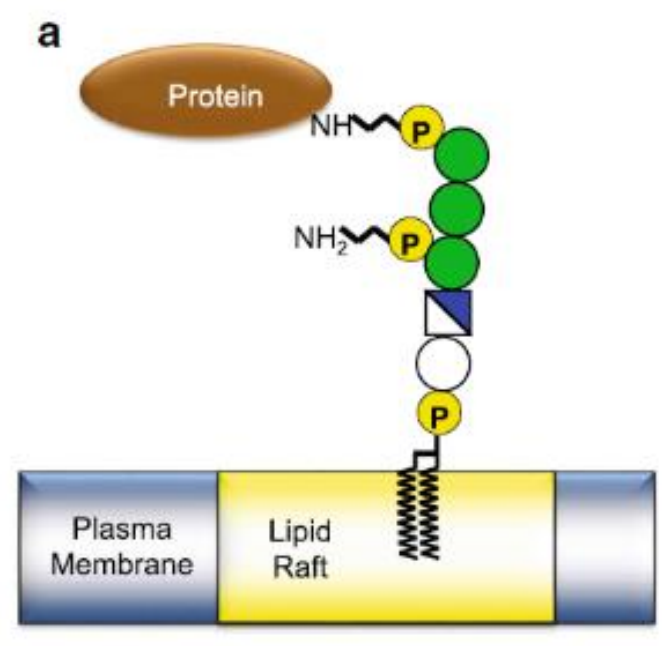

b
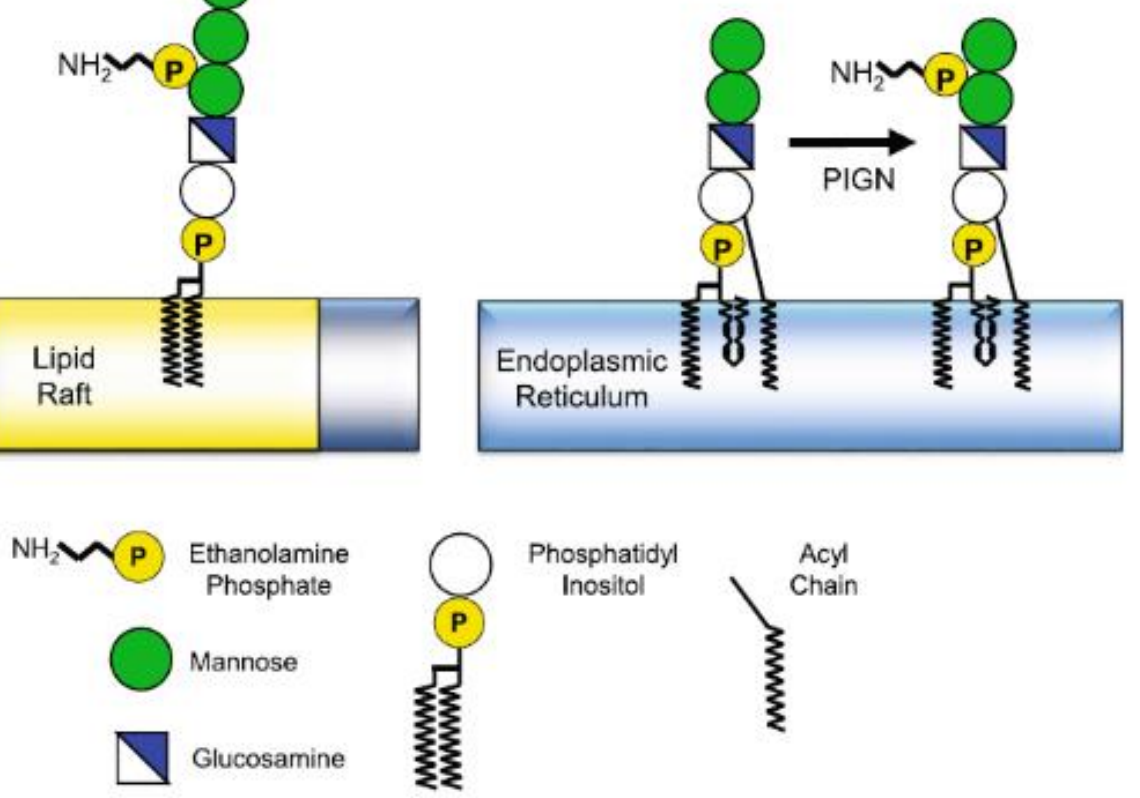

Fig. 3. Structure and synthesis of GPI. a. the core structure of the GPI anchor is a phosphatidylinositol moiety, a glucosamine moiety, three mannoses, and a ethanolamine phosphate (EtNP) on the terminal mannose. The lipid tails of PI are embedded in lipid rafts in the plasma membrane, and cell-surface proteins are bound to the terminal EtNP. Another EtNP bound to the first mannose is found in all mammalian cells. b. PIGN adds the EtNP to the first mannose during the synthesis of GPI in the endoplasmic reticulum. ${ }^{119}$

The variant PIGN:c.398T allele is predicted to result in a missense mutation, PIGN:p.T133I, in the phosphodiesterase domain. Evidence that the PIGN:p.T133I substitution partially impairs biological function was obtained by transiently transfecting PIGN-knockout HEK293 cells with a human PIGN cDNA construct designed to encode the p.T133I substitution. As demonstrated by flow cytometry, the human PIGN:p.T133I cDNA was $\sim 60 \%$ as effective as wild-type PIGN cDNA in restoring CD59 surface antigen to the PIGN-knockout cells. Available sequence information indicates that PIGN orthologs in at least 146 vertebrate species have a threonine codon at the position orthologous to codon 133 in the human and 
canine PIGN; however, at least two vertebrate species, the pig and the cape elephant shrew, have a serine codon at this position (Supplemental table at: https://static-content.springer.com/esm/art\%3A10.1007\%2Fs10048-016-05024/MediaObjects/10048 2016502 MOESM2 ESM.tif). This suggested that the hydroxyl moiety of threonine or serine might be required for normal biological function. To test this hypothesis, we transiently transfected PIGN-knockout HEK293 cells with recombinant human PIGN cDNA designed to encode either PIGN:p.T133S or PIGN:p.T133V. With both of these constructs, the restoration of CD59 surface antigen was similar to that produced by the wild-type PIGN cDNA. The apparently normal function of PIGN with a valine at position 133 indicates that hydroxyl moiety at this position is not a functional requirement. Nonetheless, the contrast in flow cytometry patterns obtained with the various cDNAs substantiates the significance of the $30-40 \%$ decrease in CD59 surface antigen restoration that was obtained with the cDNA containing the p.T133I variant.

$\mathrm{N}$-ethyl-N-nitrosourea-derived mice with a homozygous truncating splice-site mutation in Pign die in utero with developmental deficiencies including holoprosencephaly. ${ }^{132}$ Similarly, a human fetus died with a diaphragmatic hernia and many other severe developmental abnormalities, apparently due to a homozygous nullifying splice-site mutation. ${ }^{133}$ Individuals born with other homozygous or compound heterozygous PIGN mutations share a developmental deficiency syndrome known as multiple congenital anomalies-hypotonia-seizures syndrome-1 or MCAHS1 (OMIM \#614,080). The current literature describes at least 17 MCAHS1 patients from 9 families carrying 13 different PIGN 
mutations. ${ }^{118,129-131,134,135}$ These patients had neonatal hypotonia, severe developmental delays, congenital anomalies, visual impairment, hyporeflexia, tremors, and seizures. Some exhibited choreoathetosis and gait abnormalities, while others had structural brain abnormalities including delayed myelination, cortical atrophy, diffusion restriction in the globus pallidi and corticospinal tracts, and cerebellar atrophy or parenchymal loss in the vermis. ${ }^{118,131,135}$ The severity of clinical signs in MCAHS1 patients appears to correlate with the functional severity of the PIGN mutations. The most severe signs, which included multiple structural and functional developmental anomalies, occurred in a neonate with a frameshift mutation in trans with a multi-exon microdeletion. In contrast, a child with likely hypomorphic compound heterozygous missense PIGN mutations showed no dysmorphic features, moderate developmental delay, and a later onset of seizures and spastic quadriparesis. ${ }^{135}$

The cPxD described in the current report is less severe than the human and murine PIGN-associated phenotypes. In contrast to the developmental delays and early mortality seen in humans ${ }^{118,131,135}$ and mice, ${ }^{132}$ respectively, with loss of PIGN function, the first episodes of dyskinesia occurred in young-adult dogs. The dogs appeared to be normal prior to the onset of the dyskinesia and between episodes. The marked differences in severity may be due to inherent differences between species. Alternatively, the differences in severity may occur because the canine mutation encodes a product which retains a relatively greater amount of residual functional activity. This second possibility is supported by the finding that transfection of PIGN-knockout HEK293 cells with human T133I PIGN cDNA in a 
strong promoter-driven pME vector fully restored CD59 surface expression, whereas a recent report ${ }^{118}$ showed that transfection of PIGN-knockout HEK293 cells with the same strong promoter-driven vector and PIGN cDNA carrying the MCAHS1-associated S270P resulted in markedly reduced CD59 surface expression (see Fig. $2 b$ in reference ${ }^{118}$ ). Thus, it seems possible that hypomorphic mutations of human PIGN could also cause PxD or other disease phenotypes that are less severe than those of MCAHS1. Similar variability in phenotype is seen in SLC2A1 variants associated with PxD in humans. ${ }^{96,97,136}$

In summary, we have described a paroxysmal dyskinesia in dogs that most closely resembles PNKD but differs from the known human PxDs; in that, it is autosomal recessively inherited. This disease is very likely caused by a hypomorphic missense mutation in PIGN, which encodes an enzyme in the biosynthetic pathway for GPI anchors. If so, it expands the phenotypes associated with altered GPI function and suggests candidate genes to investigate in human PxD that have not had a causal mutation identified. The spontaneous canine disease could serve as an animal model to investigate the pathogenesis of $\mathrm{PxD}$ and potential therapies. Nonetheless, we cannot rule out the possibility that a variant in tight linkage disequilibrium with the PIGN:c.398C $>$ T transition is the true cause of the disease. Generation of a mouse model expressing the PIGN:c.398T variant that recapitulated the phenotype seen in dogs or the identification of other PIGN variants in dogs with $c P x D$ would help confirm that the variant is the causal and permit further studies on its effect on GPI function and the difference in phenotype with the null mutations in mice and humans. 
Supplemental Table 1a: Homozygous unique coding variants in first whole genome sequence produced with PCR-amplified libraries.

\begin{tabular}{|c|c|c|c|c|c|c|}
\hline GENE & CFA & CHR_POS & REF_BASE & VARIANT & AA_CHANGE & COVERAGE \\
\hline ACCN4 & 37 & 26025744 & $A$ & $\mathrm{C}$ & $289 D>A$ & 3 \\
\hline ACCN4 & 37 & 26025758 & $A$ & $\mathrm{C}$ & $294 T>P$ & 4 \\
\hline$A C O X L$ & 17 & 35270351 & $\mathrm{~T}$ & G & $132 V>G$ & 3 \\
\hline ACOXL & 17 & 35270356 & $\mathrm{C}$ & G & $134 \mathrm{~L}>\mathrm{V}$ & 3 \\
\hline ADAMTS15 & 5 & 4458444 & $\mathrm{~T}$ & $A$ & $787 \mathrm{~K}>\mathrm{M}$ & 6 \\
\hline AHNAK & 18 & 54109648 & G & $A$ & $3709 V>1$ & 3 \\
\hline ANXA7 & 4 & 23951430 & $\mathrm{C}$ & G & $49 A>P$ & 2 \\
\hline$A N X A 7$ & 4 & 23951436 & G & $A$ & $47 \mathrm{P}>\mathrm{S}$ & 2 \\
\hline$A N X A 7$ & 4 & 23951442 & $\mathrm{~T}$ & C & $45 \mathrm{~T}>\mathrm{A}$ & 2 \\
\hline$A N X A 7$ & 4 & 23951448 & C & $\mathrm{T}$ & $43 G>R$ & 2 \\
\hline ARHGAP2O & 5 & 22230860 & $\mathrm{C}$ & delCCC & In-Frame & 2 \\
\hline$A S P G$ & 8 & 71757312 & $\mathrm{~T}$ & G & $129 V>G$ & 2 \\
\hline ATP13A2 & 2 & 81214960 & $\mathrm{~T}$ & C & $1148 \mathrm{~F}>\mathrm{S}$ & 5 \\
\hline$B A / 2$ & 2 & 69360924 & $A$ & $\mathrm{C}$ & $271 N>T$ & 3 \\
\hline$B A / 2$ & 2 & 69360926 & $\mathrm{~T}$ & $\mathrm{C}$ & $272 S>P$ & 3 \\
\hline BEGAIN & 8 & 68796283 & $\mathrm{~T}$ & G & $191 D>A$ & 8 \\
\hline$B R W D 1$ & 31 & 34164606 & $\mathrm{C}$ & $A$ & $970 G>C$ & 2 \\
\hline $\mathrm{C} 10 \mathrm{H} 2 \mathrm{orf55}$ & 10 & 43842573 & A & $\mathrm{C}$ & $423 T>P$ & 7 \\
\hline C14H7orf60 & 14 & 52424229 & G & $A$ & $151 T>1$ & 2 \\
\hline C17H1orf56 & 17 & 60135634 & $A$ & C & $58 K>Q$ & 8 \\
\hline C2OH19orf29 & 20 & 55815800 & $\mathrm{~T}$ & $\mathrm{C}$ & $582 S>P$ & 5 \\
\hline С30H15orf61 & 30 & 31644657 & $A$ & C & $114 N>T$ & 5 \\
\hline C37H2orf47 & 37 & 9138416 & $\mathrm{~T}$ & G & $226 C>G$ & 2 \\
\hline CADM2 & 31 & 1965176 & C & $\mathrm{T}$ & $297 E>K$ & 8 \\
\hline CAPZA2 & 14 & 55809759 & C & G & $129 R>G$ & 4 \\
\hline$C C D C 40$ & 9 & 1641190 & G & C & $338 A>G$ & 3 \\
\hline$C C N D B P 1$ & 30 & 10054083 & $A$ & C & $39 E>A$ & 6 \\
\hline CD14 & 2 & 35792097 & C & $A$ & $156 G>W$ & 40 \\
\hline CDK18 & 38 & 1999372 & $A$ & C & $448 \mathrm{Q}>\mathrm{H}$ & 2 \\
\hline CEP135 & 13 & 48248017 & $A$ & G & $437 D>G$ & 5 \\
\hline CNO & 3 & 58822876 & $\mathrm{~T}$ & G & $156 V>G$ & 6 \\
\hline CNO & 3 & 58822878 & $\mathrm{~T}$ & G & $157 Y>D$ & 6 \\
\hline CRBN & 20 & 14247670 & G & $A$ & $142 E>K$ & 3 \\
\hline CSNK2A2 & 2 & 58398399 & C & G & $5 P>A$ & 2 \\
\hline CSNK2A2 & 2 & 58398400 & C & G & $5 P>R$ & 2 \\
\hline CSNK2A2 & 2 & 58398402 & $C$ & G & $6 P>A$ & 2 \\
\hline CSNK2A2 & 2 & 58398403 & C & G & $6 P>R$ & 2 \\
\hline CYP11B2 & 13 & 36877031 & $A$ & C & $239 Y>S$ & 3 \\
\hline DCTN6 & 16 & 34124786 & $\mathrm{G}$ & $\mathrm{T}$ & $97 Q>K$ & 4 \\
\hline
\end{tabular}




\begin{tabular}{|c|c|c|c|c|c|c|}
\hline GENE & CFA & CHR_POS & REF_BASE & VARIANT & AA_CHANGE & COVERAGE \\
\hline DCTNG & 16 & 34124789 & A & G & $96 S>P$ & 4 \\
\hline DIRC2 & 33 & 25910104 & $\mathrm{~T}$ & G & $298 S>A$ & 2 \\
\hline$D L A-12$ & 12 & 933665 & G & $A$ & $58 \mathrm{D}>\mathrm{N}$ & 3 \\
\hline DLL1 & 12 & 72261728 & $\mathrm{~T}$ & C & $1204 X>W$ & 3 \\
\hline DLL1 & 12 & 72261743 & $A$ & G & $1199 M>T$ & 3 \\
\hline EBF1 & 4 & 51381583 & $A$ & G & $78 Q>R$ & 8 \\
\hline EBF1 & 4 & 51381586 & G & C & $79 G>A$ & 8 \\
\hline EID1 & 30 & 15109191 & C & $A$ & $10 \mathrm{~L}>\mathrm{M}$ & 2 \\
\hline EID1 & 30 & 15109274 & C & G & $37 S>R$ & 7 \\
\hline$E S D$ & 22 & 4569705 & $T$ & C & $264 F>L$ & 3 \\
\hline FAF1 & 15 & 10425121 & G & $\mathrm{C}$ & $144 W>S$ & 5 \\
\hline FAM120C & 39 & 45943538 & $\mathrm{~T}$ & G & $7 K>Q$ & 2 \\
\hline FAM120C & 39 & 45943539 & $\mathrm{~T}$ & G & $6 \mathrm{~K}>\mathrm{N}$ & 2 \\
\hline FAM120C & 39 & 45943540 & $\mathrm{~T}$ & $A$ & $6 K>1$ & 2 \\
\hline FAM120C & 39 & 45943541 & $T$ & $A$ & $6 K>X$ & 2 \\
\hline FAM120C & 39 & 45943542 & $\mathrm{~T}$ & G & $5 E>D$ & 2 \\
\hline FAM120C & 39 & 45943543 & $T$ & $C$ & $5 E>G$ & 2 \\
\hline FAM131A & 34 & 17222704 & C & insG & FS & 2 \\
\hline FAM131A & 34 & 17222709 & $T$ & G & $405 \mathrm{~L}>\mathrm{R}$ & 2 \\
\hline FAM98A & 17 & 26677147 & G & $C$ & $236 S>C$ & 4 \\
\hline FBRSL1 & 26 & 536801 & $\mathrm{~T}$ & G & $770 D>A$ & 9 \\
\hline FFAR1 & 1 & 117257822 & $\mathrm{~T}$ & G & $39 \mathrm{~T}>\mathrm{P}$ & 3 \\
\hline$F R Y L$ & 13 & 44398730 & $\mathrm{~T}$ & $C$ & $664 D>G$ & 6 \\
\hline$F R Y L$ & 13 & 44398733 & $T$ & $C$ & $663 \mathrm{E}>\mathrm{G}$ & 6 \\
\hline FTH1 & 11 & 39037568 & $A$ & G & $14 \mathrm{H}>\mathrm{R}$ & 3 \\
\hline GALNT13 & 36 & 973660 & $\mathrm{~T}$ & $C$ & $294 L>P$ & 2 \\
\hline GEM & 29 & 39025840 & $A$ & G & $241 \mathrm{~L}>\mathrm{P}$ & 2 \\
\hline GLB1 & 23 & 3769351 & $A$ & G & $276 \mathrm{D}>\mathrm{G}$ & 6 \\
\hline GPAA1 & 13 & 37579195 & $C$ & G & $3941>M$ & 2 \\
\hline GPAA1 & 13 & 37579198 & $\mathrm{~T}$ & G & $395 \mathrm{H}>\mathrm{Q}$ & 2 \\
\hline GPAA1 & 13 & 37579201 & C & G & $396 \mathrm{~F}>\mathrm{L}$ & 2 \\
\hline GPRASP1 & 39 & 76315045 & $C$ & G & $436 R>G$ & 7 \\
\hline GPRASP1 & 39 & 76315046 & G & A & $436 R>Q$ & 7 \\
\hline GPRASP1 & 39 & 76315047 & G & ins A & FS & 7 \\
\hline GPRASP1 & 39 & 76315048 & $\mathrm{~T}$ & A & $437 W>R$ & 7 \\
\hline GPRASP1 & 39 & 76315049 & G & $A$ & $437 W>X$ & 7 \\
\hline GPRASP1 & 39 & 76315055 & $A$ & $\mathrm{~T}$ & $439 \mathrm{D}>\mathrm{V}$ & 7 \\
\hline HDAC10 & 10 & 17005973 & $\mathrm{~T}$ & $C$ & $735 S>P$ & 8 \\
\hline HDAC10 & 10 & 17005979 & $A$ & $C$ & 737T >P & 8 \\
\hline HMP19 & 4 & 38493737 & $\mathrm{~T}$ & G & $136 \mid>L$ & 10 \\
\hline HOXC13 & 27 & 1322988 & $A$ & G & $128 \mathrm{~L}>\mathrm{P}$ & 3 \\
\hline
\end{tabular}




\begin{tabular}{|c|c|c|c|c|c|c|}
\hline GENE & CFA & CHR_POS & REF_BASE & VARIANT & AA_CHANGE & COVERAGE \\
\hline$H R$ & 25 & 35131009 & $\mathrm{C}$ & insG & FS & 6 \\
\hline$H R$ & 25 & 35131010 & $A$ & $\mathrm{G}$ & $847 Q>R$ & 6 \\
\hline$H R$ & 25 & 35131013 & $A$ & G & $848 \mathrm{E}>\mathrm{G}$ & 6 \\
\hline$I L 17 B$ & 4 & 59583768 & G & $\mathrm{C}$ & $115 \mathrm{D}>\mathrm{H}$ & 8 \\
\hline IRF2BP1 & 1 & 109740090 & $\mathrm{C}$ & insG & FS & 2 \\
\hline ITGB3BP & 5 & 46682272 & $A$ & G & $19 N>D$ & 2 \\
\hline KCNA4 & 21 & 50116932 & $A$ & C & $192 V>G$ & 4 \\
\hline KCNA4 & 21 & 50116935 & $A$ & $\mathrm{C}$ & $191 V>G$ & 4 \\
\hline KCNF1 & 17 & 7752774 & C & G & $42 \mathrm{~L}>\mathrm{V}$ & 2 \\
\hline KIAA1109 & 19 & 17956232 & $\mathrm{~T}$ & $A$ & $1069 E>V$ & 5 \\
\hline KIAA1109 & 19 & 17956233 & $\mathrm{C}$ & $A$ & $1069 E>X$ & 5 \\
\hline KIF2OB & 28 & 4621592 & $A$ & G & $764 \mathrm{~T}>\mathrm{A}$ & 5 \\
\hline KIF26A & 8 & 71822968 & $\mathrm{~T}$ & C & $72 L>P$ & 3 \\
\hline LAMA4 & 12 & 68574102 & $\mathrm{~T}$ & G & $672 \mathrm{H}>\mathrm{P}$ & 5 \\
\hline LOC100682952 & 10 & 8635905 & $\mathrm{~T}$ & $\mathrm{C}$ & $1 \mathrm{M}>\mathrm{V}$ & 3 \\
\hline LOC100683298 & 20 & 54432891 & $\mathrm{~T}$ & C & $195 S>P$ & 5 \\
\hline LOC100683298 & 20 & 54432895 & $A$ & C & $196 \mathrm{~N}>\mathrm{T}$ & 5 \\
\hline LOC100685794 & 5 & 36098038 & $\mathrm{~T}$ & $A$ & $3 M>K$ & 2 \\
\hline LOC100688783 & 26 & 27216883 & $A$ & $\mathrm{~T}$ & $92 \mathrm{~T}>\mathrm{S}$ & 9 \\
\hline LOC100688783 & 26 & 27216904 & G & $A$ & $99 \mathrm{E}>\mathrm{K}$ & 9 \\
\hline LOC100855692 & 10 & 61863067 & $A$ & G & $3331>T$ & 2 \\
\hline LOC100855864 & 8 & 72736858 & $\mathrm{~T}$ & G & $108 S>A$ & 9 \\
\hline LOC100856304 & 2 & 18383718 & $\mathrm{~T}$ & G & $74 \mathrm{D}>\mathrm{A}$ & 2 \\
\hline LOC100856304 & 2 & 18383724 & $A$ & $\mathrm{~T}$ & $72 \mathrm{~L}>\mathrm{H}$ & 2 \\
\hline LOC100856304 & 2 & 18383729 & $A$ & $\mathrm{C}$ & $70 F>L$ & 2 \\
\hline LOC100856351 & 11 & 39043361 & $A$ & G & $81 C>R$ & 2 \\
\hline LOC477695 & 27 & 35133108 & $\mathrm{C}$ & $\mathrm{T}$ & $142 \mathrm{H}>\mathrm{Y}$ & 2 \\
\hline LOC487073 & 2 & 1320994 & $\mathrm{~T}$ & G & $864 V>G$ & 6 \\
\hline LOC487543 & 30 & 13354528 & $\mathrm{C}$ & G & $121 A>G$ & 2 \\
\hline LOC487670 & 30 & 39945445 & $\mathrm{C}$ & G & $535 A>P$ & 6 \\
\hline LOC487815 & 32 & 1475718 & $\mathrm{~T}$ & G & $273 Q>P$ & 5 \\
\hline LOC490390 & 7 & 39229174 & $A$ & G & $235 E>G$ & 8 \\
\hline LOC490390 & 7 & 39229176 & $\mathrm{C}$ & G & $236 Q>E$ & 8 \\
\hline LOC607594 & 25 & 24797574 & $A$ & G & $51 \mathrm{E}>\mathrm{G}$ & 5 \\
\hline LOC607594 & 25 & 24797576 & $C$ & G & $52 R>G$ & 5 \\
\hline LOC610891 & 38 & 23651499 & $\mathrm{~T}$ & G & $896 V>G$ & 2 \\
\hline LOC611902 & 17 & 37499510 & $A$ & G & $116 N>D$ & 2 \\
\hline LOC611902 & 17 & 37499511 & $A$ & G & $116 N>S$ & 2 \\
\hline LOC611902 & 17 & 37499513 & $T$ & $A$ & $117 L>\mid$ & 2 \\
\hline LONRF3 & 39 & 91106869 & $A$ & G & $125 \mathrm{E}>\mathrm{G}$ & 6 \\
\hline LPHN1 & 20 & 48380578 & $C$ & G & $1445 P>A$ & 2 \\
\hline LPHN1 & 20 & 48380578 & $C$ & G & $1445 P>A$ & 2 \\
\hline
\end{tabular}




\begin{tabular}{|c|c|c|c|c|c|c|}
\hline GENE & CFA & CHR_POS & REF_BASE & VARIANT & AA_CHANGE & COVERAGE \\
\hline LRPPRC & 10 & 46438325 & $\mathrm{~T}$ & G & $6 \mathrm{R}>\mathrm{S}$ & 2 \\
\hline$L R R C 27$ & 28 & 40298617 & A & G & $858 E>G$ & 6 \\
\hline LRRC33 & 33 & 29699324 & C & G & $682 P>A$ & 6 \\
\hline LRRC45 & 9 & 298900 & $\mathrm{~T}$ & G & $365 D>A$ & 2 \\
\hline MAPKAPK2 & 7 & 5901211 & A & $\mathrm{C}$ & $241 T>P$ & 9 \\
\hline$M B I P$ & 8 & 14886709 & $\mathrm{~T}$ & C & $322 S>G$ & 2 \\
\hline MEAF6 & 15 & 4947987 & $\mathrm{~T}$ & G & $81 V>G$ & 6 \\
\hline$M R G P R G$ & 18 & 47136179 & C & G & $100 R>P$ & 10 \\
\hline MRPL46 & 3 & 51702661 & $\mathrm{~T}$ & G & $184 \mathrm{~T}>\mathrm{P}$ & 3 \\
\hline MYO5A & 30 & 18052103 & C & G & $952 R>P$ & 2 \\
\hline MYO5A & 30 & 18052106 & G & $\mathrm{C}$ & $951 A>G$ & 2 \\
\hline NF1 & 9 & 41505776 & $\mathrm{~T}$ & C & $2212 D>G$ & 5 \\
\hline NFKBIZ & 33 & 8125937 & A & G & $72 K>R$ & 2 \\
\hline NFKBIZ & 33 & 8125951 & $\mathrm{~T}$ & C & $77 S>P$ & 3 \\
\hline NFKBIZ & 33 & 8125958 & $\mathrm{~T}$ & C & $79 F>S$ & 3 \\
\hline NFKBIZ & 33 & 8125965 & G & C & $81 R>S$ & 3 \\
\hline NFKBIZ & 33 & 8125967 & G & $C$ & $82 \mathrm{G}>\mathrm{A}$ & 3 \\
\hline NID1 & 4 & 3985012 & C & G & $720 S>R$ & 3 \\
\hline NID1 & 4 & 3985014 & A & $C$ & $721 \mathrm{H}>\mathrm{P}$ & 3 \\
\hline NUDT6 & 19 & 17375074 & A & G & $131 E>G$ & 3 \\
\hline PALM & 20 & 57855581 & $\mathrm{~T}$ & G & $144 T>P$ & 7 \\
\hline PARP14 & 33 & 25782161 & A & $C$ & $908 \mathrm{E}>\mathrm{A}$ & 8 \\
\hline PIGN & 1 & 14705240 & C & $\mathrm{T}$ & 133T>I & 16 \\
\hline PKHD1L1 & 13 & 10054619 & $\mathrm{~T}$ & G & $3805 C>W$ & 11 \\
\hline PKHD1L1 & 13 & 10054621 & C & G & $3806 A>G$ & 11 \\
\hline PLBD2 & 26 & 10709971 & C & $A$ & $134 L>\mid$ & 2 \\
\hline PLBD2 & 26 & 10709975 & $\mathrm{~T}$ & A & $135 F>Y$ & 2 \\
\hline PLBD2 & 26 & 10709977 & $\mathrm{~T}$ & $A$ & $136 \mathrm{~L}>\mathrm{M}$ & 2 \\
\hline PLBD2 & 26 & 10709981 & C & A & $137 \mathrm{P}>\mathrm{H}$ & 2 \\
\hline PLEC & 13 & 37450827 & $\mathrm{~T}$ & G & $4573 Y>S$ & 5 \\
\hline$P M L$ & 30 & 37265646 & $C$ & $\mathrm{~T}$ & $500 R>W$ & 47 \\
\hline PPM1E & 9 & 33350900 & G & $A$ & $96 G>D$ & 3 \\
\hline PRR12 & 1 & 106921117 & $A$ & C & $1554 C>G$ & 7 \\
\hline PRSS33 & 6 & 38320579 & G & C & $43 R>G$ & 6 \\
\hline$R A P 1 A$ & 17 & 63742031 & C & $A$ & $25 Q>H$ & 2 \\
\hline$R A P 1 A$ & 17 & 63742032 & $\mathrm{~T}$ & G & $25 Q>P$ & 2 \\
\hline RBM10 & 39 & 40762898 & G & C & $145 G>R$ & 2 \\
\hline RBM10 & 39 & 40762904 & G & C & $147 G>R$ & 2 \\
\hline RBM10 & 39 & 40762907 & G & C & $148 G>R$ & 2 \\
\hline RBM10 & 39 & 40762913 & G & C & $150 G>R$ & 2 \\
\hline RFWD2 & 7 & 23321045 & $A$ & G & $114 K>E$ & 5 \\
\hline RIN2 & 24 & 3371828 & $\mathrm{~T}$ & G & $387 \mathrm{~K}>\mathrm{N}$ & 9 \\
\hline
\end{tabular}




\begin{tabular}{|c|c|c|c|c|c|c|}
\hline GENE & CFA & CHR_POS & REF_BASE & VARIANT & AA_CHANGE & COVERAGE \\
\hline RNASEH2B & 22 & 967992 & G & insA & FS & 6 \\
\hline ROR2 & 1 & 95278502 & $\mathrm{C}$ & $\mathrm{G}$ & $464 A>G$ & 3 \\
\hline$R R P 1 B$ & 31 & 37663897 & G & inst & FS & 2 \\
\hline RSPH4A & 1 & 57074175 & $\mathrm{~T}$ & $A$ & $572 D>E$ & 3 \\
\hline RXFP3 & 4 & 73873384 & $A$ & $\mathrm{C}$ & $243 V>G$ & 4 \\
\hline S100PBP & 2 & 68499452 & $\mathrm{~T}$ & G & $400 T>P$ & 3 \\
\hline SCNN1B & 6 & 22462890 & C & G & $344 A>P$ & 5 \\
\hline SCNN1B & 6 & 22462892 & $\mathrm{~T}$ & G & $343 N>T$ & 5 \\
\hline SEPT1 & 6 & 17708699 & $A$ & G & $117 R>G$ & 4 \\
\hline SEPT1 & 6 & 17708702 & $\mathrm{C}$ & G & $118 \mathrm{P}>\mathrm{A}$ & 4 \\
\hline SLC12A3 & 2 & 59437916 & $\mathrm{~T}$ & $\mathrm{C}$ & $496 K>E$ & 12 \\
\hline SLC25A46 & 3 & 1718383 & A & C & $35 \mathrm{~L}>\mathrm{R}$ & 4 \\
\hline SLC25A46 & 3 & 1718389 & $\mathrm{C}$ & $A$ & $33 G>V$ & 4 \\
\hline SLC25A46 & 3 & 1718392 & $\mathrm{C}$ & $A$ & $32 \mathrm{G}>\mathrm{V}$ & 4 \\
\hline SLC25A46 & 3 & 1718393 & $\mathrm{C}$ & $\mathrm{T}$ & $32 \mathrm{G}>\mathrm{R}$ & 4 \\
\hline SLC29A3 & 4 & 22098019 & $A$ & ins $C$ & FS & 2 \\
\hline SLC30A1 & 7 & 9959075 & $C$ & G & $104 Q>H$ & 2 \\
\hline SLC35F5 & 19 & 35270909 & $C$ & $A$ & $98 \mathrm{~N}>\mathrm{K}$ & 5 \\
\hline$S L K$ & 28 & 16312240 & $\mathrm{~T}$ & $\mathrm{C}$ & $146 L>S$ & 2 \\
\hline SMAD6 & 30 & 31013728 & $A$ & $C$ & $485 T>P$ & 5 \\
\hline SMAD6 & 30 & 31013731 & $\mathrm{~T}$ & $C$ & $486 S>P$ & 5 \\
\hline sox3o & 4 & 52587962 & $A$ & G & $203 D>G$ & 10 \\
\hline SP7 & 27 & 1886597 & G & $\mathrm{C}$ & $341 E>Q$ & 3 \\
\hline SPACA1 & 12 & 47450050 & $C$ & G & $19 L>V$ & 13 \\
\hline SPATA21 & 2 & 81306486 & G & $\mathrm{C}$ & $433 E>Q$ & 4 \\
\hline SPEN & 2 & 81687640 & $A$ & C & $3227 V>G$ & 4 \\
\hline SSC5D & 1 & 102196945 & G & ins $A$ & FS & 2 \\
\hline SSC5D & 1 & 102196950 & $C$ & delC & FS & 2 \\
\hline STOML1 & 30 & 37226705 & $C$ & $\mathrm{~T}$ & $127 \mathrm{~V}>\mathrm{M}$ & 23 \\
\hline SYNJ1 & 31 & 27356222 & G & $A$ & $412 A>V$ & 6 \\
\hline TAF6L & 18 & 53899260 & $\mathrm{~T}$ & G & $469 T>P$ & 5 \\
\hline TAOK1 & 9 & 43594263 & $A$ & $\mathrm{C}$ & $175 M>L$ & 6 \\
\hline$T B X A 2 R$ & 20 & 55824993 & $C$ & G & $195 A>G$ & 2 \\
\hline TENC1 & 27 & 2099162 & $C$ & G & $1053 E>D$ & 2 \\
\hline TGDS & 22 & 45386237 & $\mathrm{~T}$ & G & $295 R>S$ & 3 \\
\hline TGFA & 10 & 68910846 & $C$ & G & $422 \mathrm{~V}>\mathrm{L}$ & 5 \\
\hline THEM4 & 17 & 60925021 & $\mathrm{~T}$ & G & $24 \mathrm{H}>\mathrm{P}$ & 3 \\
\hline TJP3 & 20 & 55722132 & $\mathrm{~T}$ & G & $789 D>A$ & 4 \\
\hline TMCC2 & 38 & 1771057 & G & $C$ & $443 A>P$ & 13 \\
\hline TMEM151A & 18 & 51010388 & G & $C$ & $145 A>G$ & 4 \\
\hline TMPRSS9 & 20 & 56680769 & $A$ & G & $310 L>P$ & 2 \\
\hline TOММ20 & 4 & 4800927 & $\mathrm{C}$ & $\mathrm{T}$ & $136 A>V$ & 2 \\
\hline
\end{tabular}




\begin{tabular}{lllllll}
\hline GENE & CFA & CHR_POS & REF_BASE & VARIANT & AA_CHANGE & COVERAGE \\
\hline TRIM23 & 2 & 51353677 & $\mathrm{~T}$ & $\mathrm{G}$ & $61 \mathrm{~T}>\mathrm{P}$ & 3 \\
TRIOBP & 10 & 26884008 & $\mathrm{C}$ & $\mathrm{A}$ & $1584 \mathrm{~A}>\mathrm{S}$ & 12 \\
TRIOBP & 10 & 26884010 & $\mathrm{C}$ & $\mathrm{G}$ & $1583 \mathrm{G}>\mathrm{A}$ & 12 \\
TRIOBP & 10 & 26884011 & $\mathrm{C}$ & $\mathrm{T}$ & $1583 \mathrm{G}>\mathrm{S}$ & 12 \\
TRIOBP & 10 & 26884028 & $\mathrm{G}$ & $\mathrm{A}$ & $1577 \mathrm{P}>\mathrm{L}$ & 2 \\
TRMT1 & 20 & 49101235 & $\mathrm{C}$ & $\mathrm{G}$ & $29 \mathrm{Q}>\mathrm{E}$ & 11 \\
UCHL1 & 3 & 71410964 & $\mathrm{~A}$ & $\mathrm{~T}$ & $3 \mathrm{~L}>\mathrm{H}$ & 2 \\
ULK3 & 30 & 37904557 & $\mathrm{~A}$ & $\mathrm{G}$ & $23 \mathrm{~L}>\mathrm{P}$ & 6 \\
USP25 & 31 & 12652955 & $\mathrm{~A}$ & $\mathrm{G}$ & $722 \mathrm{R}>\mathrm{G}$ & 3 \\
USP34 & 10 & 61289706 & $\mathrm{C}$ & $\mathrm{G}$ & $2885 \mathrm{~A}>\mathrm{P}$ & 5 \\
UTRN & 1 & 35876816 & $\mathrm{~A}$ & $\mathrm{C}$ & $750 \mathrm{Q}>\mathrm{H}$ & 3 \\
VANGL2 & 38 & 21828498 & $\mathrm{C}$ & $\mathrm{G}$ & $89 \mathrm{G}>\mathrm{R}$ & 10 \\
VANGL2 & 38 & 21828499 & $\mathrm{C}$ & $\mathrm{G}$ & $88 \mathrm{~K}>\mathrm{N}$ & 10 \\
VANGL2 & 38 & 21828500 & $\mathrm{~T}$ & $\mathrm{C}$ & $88 \mathrm{~K}>\mathrm{R}$ & 10 \\
VIM & 2 & 19671672 & $\mathrm{C}$ & $\mathrm{T}$ & $457 \mathrm{E}>\mathrm{K}$ & 5 \\
WDR36 & 3 & 1490475 & $\mathrm{~T}$ & $\mathrm{C}$ & $75 \mathrm{R}>\mathrm{G}$ & 5 \\
WNT5B & 27 & 43670032 & $\mathrm{C}$ & $\mathrm{A}$ & $168 \mathrm{Y}>\mathrm{X}$ & 3 \\
YARS2 & 27 & 16139787 & $\mathrm{G}$ & $\mathrm{A}$ & $97 \mathrm{G}>\mathrm{S}$ & 9 \\
YWHAE & 9 & 45512480 & $\mathrm{~T}$ & $\mathrm{C}$ & $235 \mathrm{M}>\mathrm{V}$ & 3 \\
ZBTB4 & 5 & 32388310 & $\mathrm{~T}$ & $\mathrm{C}$ & $577 \mathrm{I}>\mathrm{V}$ & 2 \\
ZC3H6 & 17 & 36646542 & $\mathrm{C}$ & $\mathrm{A}$ & $67 \mathrm{H}>\mathrm{N}$ & 2 \\
ZC3H6 & 17 & 36646543 & $\mathrm{~A}$ & $\mathrm{G}$ & $67 \mathrm{H}>\mathrm{R}$ & 2 \\
ZC3H6 & 17 & 36646544 & $\mathrm{C}$ & $\mathrm{A}$ & $67 \mathrm{H}>\mathrm{Q}$ & 2 \\
\hline & & & & & &
\end{tabular}


Supplemental Table 1b: Homozygous unique coding variants in second whole genome sequence produced with PCR-free libraries.

\begin{tabular}{|c|c|c|c|c|c|c|}
\hline GENE & CFA & CHR_POS & REF_BASE & VARIANT & AA_CHANGE & COVERAGE \\
\hline ACTR5 & 24 & 27154461 & $\mathrm{C}$ & G & $55 A>G$ & 3 \\
\hline ACTR5 & 24 & 27154463 & $\mathrm{~T}$ & G & $56 C>G$ & 3 \\
\hline ADAMTS19 & 11 & 18273233 & G & delG & FS & 2 \\
\hline$A P O B$ & 17 & 15888601 & G & A & $1560 T>M$ & 19 \\
\hline ARHGEF26 & 23 & 48250284 & $\mathrm{G}$ & $\mathrm{C}$ & $20 R>P$ & 3 \\
\hline ARHGEF26 & 23 & 48250289 & G & $A$ & $22 G>S$ & 3 \\
\hline ATP8B3 & 20 & 57160284 & G & C & $1394 R>S$ & 10 \\
\hline C18H11orf35 & 18 & 25664137 & C & G & $739 G>R$ & 3 \\
\hline C28H10orf82 & 28 & 27224783 & G & $\mathrm{T}$ & $152 Y>X$ & 28 \\
\hline С3OH15orf39 & 30 & 38175075 & $\mathrm{C}$ & delCCGGCA & In-Frame & 11 \\
\hline C4H1orf96 & 4 & 9878498 & G & delGAGGCGGCG & In-Frame & 8 \\
\hline CABLES2 & 24 & 46375466 & $\mathrm{~T}$ & G & $138 \mathrm{~T}>\mathrm{P}$ & 4 \\
\hline CNNM3 & 10 & 45154194 & G & C & $147 \mathrm{P}>\mathrm{R}$ & 2 \\
\hline CSN1S1 & 13 & 59430621 & $\mathrm{~T}$ & G & $153 D>E$ & 26 \\
\hline DFNB31 & 11 & 68699676 & $C$ & delC & FS & 2 \\
\hline$E C H D C 2$ & 5 & 55972146 & G & A & $48 R>Q$ & 2 \\
\hline FAM45A & 28 & 29346298 & $A$ & $\mathrm{G}$ & $6 S>G$ & 2 \\
\hline FAM45A & 28 & 29346301 & G & insG & FS & 2 \\
\hline FAM73A & 6 & 69026458 & $\mathrm{G}$ & A & $527 \mathrm{~L}>\mathrm{F}$ & 20 \\
\hline FBXO45 & 33 & 29635933 & $A$ & delAT & FS & 2 \\
\hline FBX045 & 33 & 29635937 & $\mathrm{~T}$ & insT & FS & 2 \\
\hline GPR83 & 21 & 6536825 & C & delCTT & In-Frame & 2 \\
\hline GTF3C1 & 6 & 19168295 & $\mathrm{G}$ & A & $1639 \mathrm{E}>\mathrm{K}$ & 13 \\
\hline JMJD8 & 6 & 39891999 & G & delGTGGCGCCGGTGCTGC & FS & 4 \\
\hline KCNQ3 & 13 & 29062325 & $\mathrm{~T}$ & G & $16 \mathrm{~N}>\mathrm{T}$ & 3 \\
\hline KIF12 & 11 & 68368122 & G & $A$ & $81 \mathrm{~A}>\mathrm{V}$ & 13 \\
\hline LATS1 & 1 & 40446218 & $\mathrm{~T}$ & C & $3451>V$ & 11 \\
\hline LEPREL2 & 27 & 38194601 & G & C & $101 S>W$ & 3 \\
\hline LOC100682747 & 14 & 11647458 & G & C & $182 \mathrm{E}>\mathrm{Q}$ & 8 \\
\hline LOC100682857 & 16 & 22212954 & $A$ & $\mathrm{~T}$ & $235 \mathrm{H}>\mathrm{L}$ & 2 \\
\hline LOC100682857 & 16 & 22212957 & $\mathrm{~T}$ & delTCG & In-Frame & 2 \\
\hline LOC100684233 & 12 & 36497100 & $\mathrm{~T}$ & delTTTTT & Splice & 7 \\
\hline LOC100684399 & 35 & 765733 & G & A & $254 G>R$ & 5 \\
\hline LOC100688053 & 6 & 53880544 & C & insT & FS & 14 \\
\hline LOC100855863 & 38 & 22151133 & C & G & $589 A>G$ & 4 \\
\hline LOC475279 & 14 & 45015191 & $A$ & $C$ & $445 V>G$ & 4 \\
\hline LOC484210 & 1 & 98449334 & G & delG & FS & 4 \\
\hline LOC484210 & 1 & 98455777 & $\mathrm{C}$ & G & $1228 \mathrm{~A}>\mathrm{P}$ & 3 \\
\hline
\end{tabular}




\begin{tabular}{|c|c|c|c|c|c|c|}
\hline GENE & CFA & CHR_POS & REF_BASE & VARIANT & AA_CHANGE & COVERAGE \\
\hline LOC485980 & 24 & 47296889 & $\mathrm{C}$ & delCC & FS & 3 \\
\hline LOC489893 & 6 & 14726554 & C & G & $1164 R>P$ & 2 \\
\hline LOC610174 & 15 & 12776779 & $A$ & $\operatorname{del} A$ & FS & 2 \\
\hline LOC610174 & 15 & 12776785 & $A$ & delAGACGG & In-Frame & 2 \\
\hline LOC612378 & 6 & 57025306 & C & G & $147 S>R$ & 2 \\
\hline LOC612378 & 6 & 57025307 & $T$ & $A$ & $148 \mathrm{~S}>\mathrm{T}$ & 2 \\
\hline$L O R$ & 17 & 61995003 & $T$ & G & $229 S>A$ & 4 \\
\hline$\angle O R$ & 17 & 61995004 & C & G & $229 S>C$ & 4 \\
\hline$L O R$ & 17 & 61995153 & $T$ & G & $279 C>G$ & 2 \\
\hline МАРЗК15 & 39 & 15465777 & $T$ & C & $29 E>G$ & 2 \\
\hline MAST3 & 20 & 44878332 & $A$ & C & $1 \mathrm{M}>\mathrm{R}$ & 4 \\
\hline MIER2 & 20 & 58064321 & $\mathrm{C}$ & $A$ & $245 R>S$ & 6 \\
\hline$O D Z 1$ & 39 & 96111976 & $A$ & G & $193 L>P$ & 3 \\
\hline PHLPP1 & 1 & 14234138 & $\mathrm{~T}$ & G & $31 E>A$ & 2 \\
\hline PIGN & 1 & 14705240 & C & $T$ & $133 \mathrm{~T}>1$ & 16 \\
\hline PKD1L3 & 5 & 77754396 & G & delG & FS & 23 \\
\hline PLK5 & 20 & 57343915 & $A$ & G & $11 S>P$ & 5 \\
\hline PRDM12 & 9 & 53284486 & G & insGCG & In-Frame & 5 \\
\hline PRR24 & 1 & 108705031 & $\mathrm{~T}$ & delT & FS & 3 \\
\hline PRR24 & 1 & 108705036 & $T$ & $\mathrm{C}$ & $20 N>S$ & 3 \\
\hline RAB12 & 7 & 74780877 & G & $A$ & $87 \mathrm{~A}>\mathrm{T}$ & 12 \\
\hline RIN3 & 8 & 1777689 & C & $\mathrm{T}$ & $319 P>L$ & 5 \\
\hline S1PR5 & 20 & 50644336 & G & C & $240 A>P$ & 2 \\
\hline SHISA2 & 25 & 13472951 & $A$ & delA & FS & 2 \\
\hline SHISA2 & 25 & 13472952 & $A$ & $T$ & $382 N>1$ & 2 \\
\hline TMEM70 & 29 & 22480075 & $\mathrm{~T}$ & G & $105 C>G$ & 6 \\
\hline TRIM44 & 18 & 32351165 & G & delGGGCGG & In-Frame & 6 \\
\hline ТTC39A & 15 & 9939623 & $A$ & $\mathrm{C}$ & $285 Y>S$ & 4 \\
\hline ZMIZ2 & 99 & 264737 & G & insC & FS & 2 \\
\hline
\end{tabular}




\section{Chapter 3}

\section{Australian Cattle Dogs with Neuronal Ceroid Lipofuscinosis are Homozygous for a CLN5 Nonsense mutation Previously Identified in Border Collies}

\section{Abstract}

Young adult Australian Cattle Dogs have been diagnosed with neuronal ceroid lipofuscinosis (NCL), a fatal neurodegenerative disease. We characterized the Australian Cattle Dog form of NCL and determined its genetic cause. Tissues from 4 Australian Cattle Dogs with NCL-like clinical signs and buccal swabs from both parents of a fifth affected breed member, plus archived DNA samples from 712 individual dogs were used for this study. The tissue samples were examined by fluorescence, electron, and imunohistochemical microscopy. A whole-genome sequence was generated for 1 affected dog and a TaqMan allelic discrimination was used for genotyping. The accumulation of autofluorescent cytoplasmic storage material with characteristic ultrastructure in tissues from the 4 affected dogs supported the NCL diagnosis. The whole-genome sequence contained a homozygous nonsense mutation: CLN5:C.619C>T. All 4 DNA samples from clinically affected dogs tested homozygous for the variant allele. Both parents of the fifth affected dog were heterozygotes. Archived DNA samples from 346 Australian Cattle Dogs, 188 Border Collies, and 177 dogs of other breeds were homozygous for the reference allele. One archived Australian Cattle Dog sample was from a heterozygote. The homozygous CLN5 nonsense mutation is almost 
certainly causal because the same mutation had been previously reported to cause a similar form of NCL in Border Collies. The detection of this mutation allows testing to confirm the NCL diagnosis in this breed.

\section{Introduction}

The neuronal ceroid lipofuscinoses (NCLs) are fatal progressive neurodegenerative diseases characterized by the accumulation of autofluorescent lysosomal storage material within the brain, the retina, and other tissues. Mutations in 13 different genes have been found to cause various forms of NCL in humans. ${ }^{137}$ This disease has been reported in a variety of wild and domestic animals including the dog. ${ }^{138,139}$ At least 10 DNA sequence variants from 8 different genes have been identified as the molecular genetic causes for NCLs in dogs (Table 1). ${ }^{51,52,117,140-}$ 148 Until recently, all of the previously identified NCL-causing sequence variants in dogs have been reported to occur within members of single breeds, although there are 2 examples of different NCL-causing alleles segregating independently in the same breed: mutations in PPT1 and TPP1 have been associated with different forms of NCL in Daschunds ${ }^{140,141}$ and mutations in CLN6 and CLN8 have been found in different NCLs of Australian Shepherds. ${ }^{52,144}$ Faller et al. ${ }^{51}$ were the first to report a previously identified NCL-causing mutation in a second dog breed when they described NCL in Chihuahua siblings that were homozygous for a single-base deletion and frameshift that had already been reported in a Chinese Crested Dog with NCL. ${ }^{117}$ 
Still to be identified are the molecular genetic causes of the previously reported NCLs in other breeds, such as the Australian Cattle Dog (also known as Blue Heeler), ${ }^{149}$ Cocker Spaniel, ${ }^{150}$ Dalmatian, ${ }^{151}$ Labrador Retriever, ${ }^{152}$ Miniature Schnauzer, ${ }^{153}$ Polish Lowland Sheepdog, ${ }^{154}$ and Saluki. ${ }^{155}$ Mutations causing NCL are likely to be segregating in additional breeds for which the disease has not yet been reported. We had an opportunity to analyze biological samples from young adult Australian Cattle Dogs with NCL-like clinical signs. Herein, we describe the disease phenotype and report that its molecular genetic cause is identical to a mutation previously reported as the cause of NCL in Border Collies. ${ }^{142}$ Thus, ours is the second report that a NCL-causing mutation is present in a dog breed other than that of the original discovery.

Table 1. Breed distribution of canine NCL-associated mutations.

\begin{tabular}{|c|c|c|c|c|}
\hline Disease & Gene & Mutation & Amino Acid Sequence & Affected Dog Breed \\
\hline CLN1 & PPT1 & PPT1:c.736_737insC & p.F246Lfs*29 & Dachshund $^{140}$ \\
\hline CLN2 & TPP1 & TPP1:c.325delC & p.A108Pfs*6 & Dachshund $^{141}$ \\
\hline CLN5 & CLN5 & CLN5:C.619C>T & p.Q207X & Border Collie ${ }^{142}$ \\
\hline CLN5 & CLN5 & CLN5:c.934_935delAG & CLN5:p.E312Vfs*6 & Golden Retriever $r^{143}$ \\
\hline CLN6 & CLN6 & CLN6:C.829T>C & p.W277R & Australian Shepherd ${ }^{144}$ \\
\hline CLN7 & MFSD8 & MFSD8c.843delT & p.F282Lfs*13 & $\begin{array}{l}\text { Chinese Crested Dog, } \\
\text { Chihuahua }^{117}\end{array}$ \\
\hline CLN8 & CLN8 & CLN8:C.491T>C & p.L164P & English Setter ${ }^{145}$ \\
\hline CLN8 & CLN8 & CLN8:C.585G>A & p.W195X & Australian Shepherd ${ }^{52}$ \\
\hline CLN10 & CTSD & CTSD:C.597G>A & p.M199I & American Bulldog ${ }^{146}$ \\
\hline CLN12 & ATP13A2 & ATP13A2:c.1623delG & p.P541fs*56 & Tibetan Terrier ${ }^{147,148}$ \\
\hline
\end{tabular}



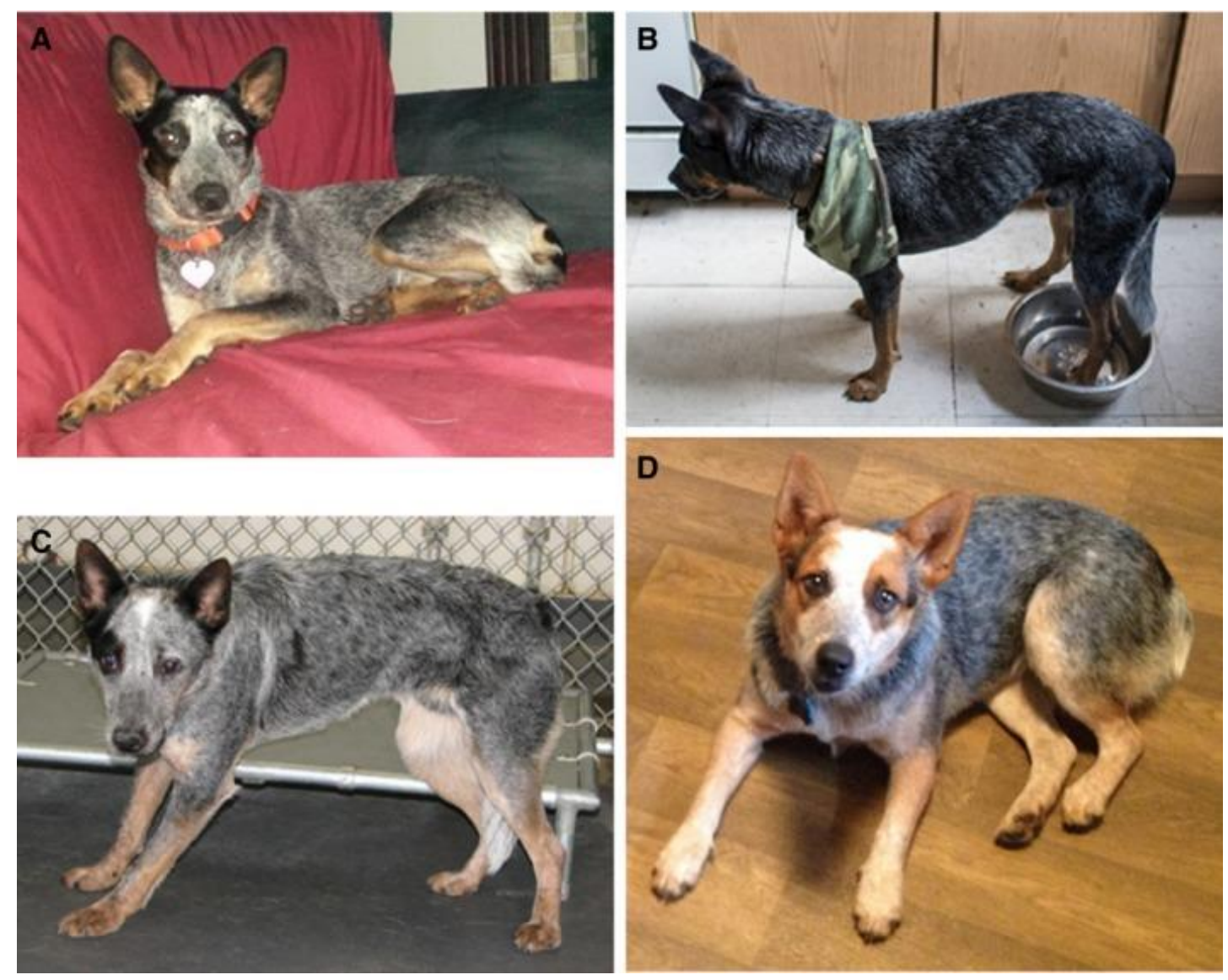

Fig. 1. Photographs of four of the subject Australian Cattle Dogs. (A) Dog A from near St. Louis euthanized in 2014, (B) Dog B from Chicago euthanized in 2014. (C) Dog C from Seattle euthanized in 2007. (D) Dog E from Alabama euthanized in 2015.

\section{Materials and Methods}

\section{Subject Australian Cattle Dogs}

Five dogs were evaluated for this study. Although pedigree information was not available for all dogs, all were believed by their owners to be purebred Australian Cattle Dogs, and according to their veterinarians, all had physical characteristics that were typical of purebred Australian Cattle Dogs (Fig. 1). Australian Cattle Dog A, a 15-month-old spayed female (Fig. 1A), was referred to the Veterinary Specialty Services Neurology Department near St. Louis for an acute onset of 
seizures and blindness in 2014. Approximately 2 weeks before presentation, the dog had experienced its first tonic-clonic seizure. The dog was reportedly completely blind in the postictal period. Partial return of vision occurred within 24 hours, but the dog never regained full vision. Over the next few days, the dog developed persistent focal seizures and "fly biting". After the initial seizure, the dog circles constantly when not sedated. The dog had been evaluated by the local veterinarian who initiated the treatment with phenobarbital. There was no improvement with phenobarbital treatment and her care was transferred to the Neurology Department at Veterinary Specialty Services. The dog had been obtained from a breeder at a young age, but pedigree records and breeder contact information were lost, and it was not possible to locate closely related dogs. Although the owners originally described no visual deficits before the first seizure, they mentioned that the dog had once been quite athletic, but recently had not been successful playing Frisbee or fetch. The dog exhibited decreased coordination that included clumsiness and bumping into objects. Starting at approximately 1 year of age, the dog began to display signs of anxiety, particularly when encountering other dogs or unfamiliar people.

The physical examination was limited because $\operatorname{dog} \mathrm{A}$ was extremely disoriented and hypersensitive to any auditory or tactile stimulation. With mild restraint, the dog would have severe myoclonic jerks. Cranial nerve examination identified a bilaterally absent menace reflex, but the remainder of the cranial nerve evaluation was normal. The dog was ambulatory with mild tetraparesis, proprioceptive ataxia, and delayed general proprioception in all 4 limbs. Dog A had intact segmental 
reflexes. A CBC and serum biochemistry analyses were unremarkable. Magnetic resonance imaging identified diffuse brain atrophy indicated by widening of the cerebral cortical sulci, diffuse dilatation of the ventricular system, subjectively decreased size of the interthalamic adhesion, and increased cerebrospinal fluid (CSF) surrounding the folia of the cerebellum (Fig. 2). Because of the severity of the clinical signs, Dog A was euthanized the day after advanced diagnostic procedures were performed.

Australian Cattle Dog B, a neutered male (Fig. 1B), presented to the Chicago Veterinary Neurology and Neurosurgery Group in 2014 at approximately 18 months of age for evaluation of progressive neurologic changes and a recent severe seizure. The onset of signs occurred at approximately 12 months of age with periodic episodes of severe anxiety, particularly in response to loud noises. The severe anxiety persisted throughout the course of the disease. Over time, the dog's ability to recognize and respond to commands progressively decreased. As the disease progressed, other signs exhibited by Dog B included compulsive repetitive scanning of its surroundings, circling, loss of ability to navigate stairs, tremors, loss of coordination, visual impairment in both dim and bright light, periods of trance-like behavior, aggression, ataxia, and clumsiness including increased bumping into obstacles. At the time of the examination, the dog reacted aggressively to any attempts to control its head or body. Because of progressing neurological signs that did not abate with zonisamide and diazepam treatment, dog B was euthanized at approximately 20 months of age and a necropsy was 
performed to collect tissues as described below. No pedigree information was available for this dog.
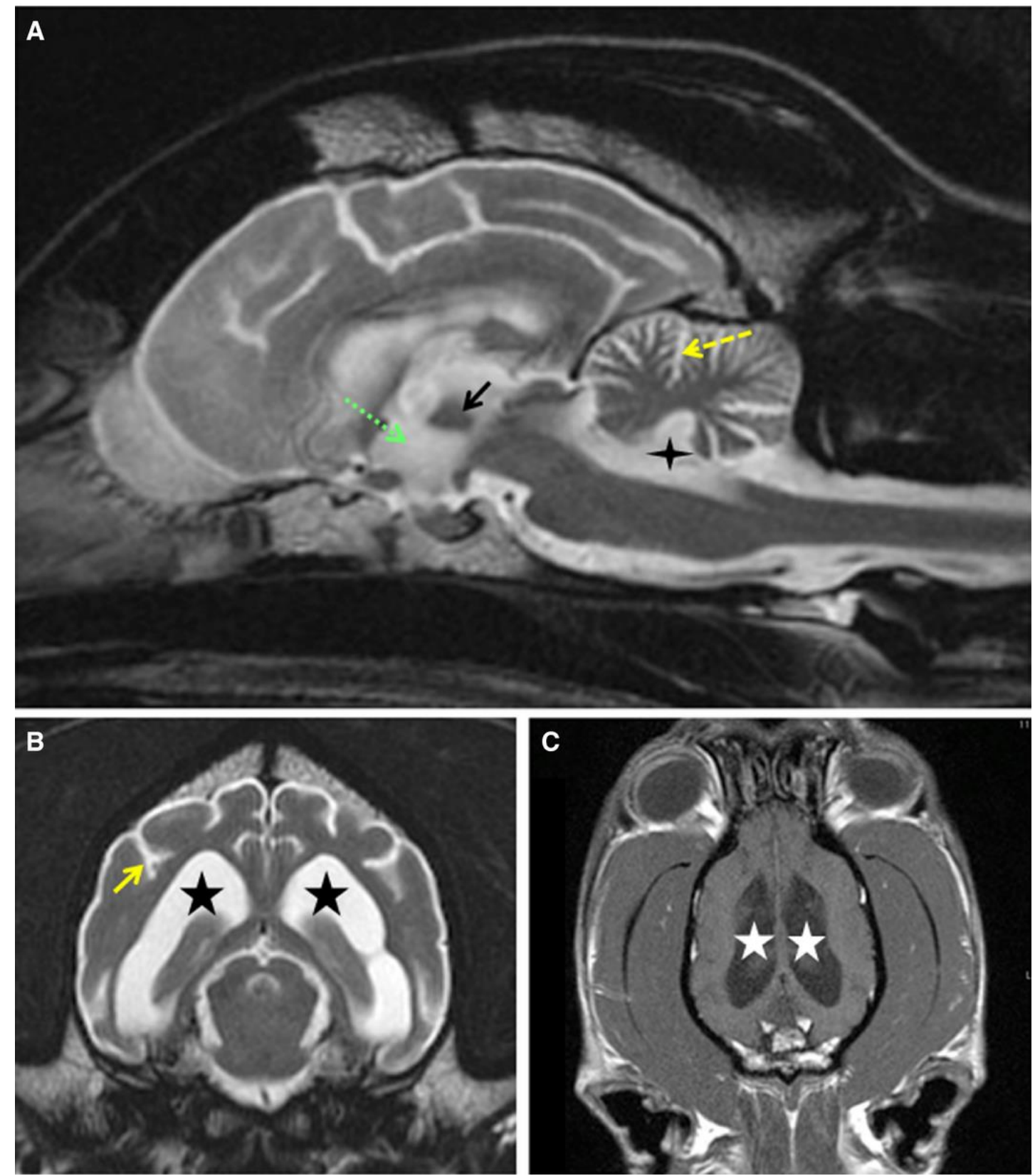

Fig. 2. T2-weighted MRI from Dog $A$ in the sagittal $(\mathbf{A})$ and transverse $(\mathbf{B})$ planes. From Dog $A, T 1$ weighted post-contrast MRI in the dorsal plane $(\mathbf{C})$. CSF is white and brain parenchyma gray in the T2-weighted images. CSF is dark gray in the T1-weighted image. Diffuse brain atrophy is indicated by atrophy of the interthalamic adhesion (black arrow in A) dilatation of the 3rd (green dashed arrow in A) 4th (4-point star in A), and lateral ventricles (5-point stars in B and C), widening of the sulci of the cerebral cortex (solid yellow arrow in B), and increased CSF surrounding the folia of the cerebellum (yellow dashed arrow in A). 
Australian Cattle Dog C (Fig. 1C) had been tentatively diagnosed with NCL in Pullman, Washington, in 2007. A DNA sample was received at the University of Missouri at that time and archived. The owners reported that during the 6 months before presentation, Dog $\mathrm{C}$ had begun showing abnormal behavior, including apparent incoordination, trance-like episodes, increasing fear of other dogs, and aggression towards the owners. At examination Dog $\mathrm{C}$ was disoriented, was overreactive to normal auditory and tactile stimuli, and had an intermittent left head tilt. Static knuckling was delayed in all 4 limbs, but no paresis was noted during observation of the gait. Tetra-ataxia also was noted, especially during pivoting. The menace response was absent bilaterally, but pupillary light reflexes were normal. Cerebrospinal fluid analysis disclosed a normal protein concentration $(12 \mathrm{mg} / \mathrm{dL}$; reference range, $<25 \mathrm{mg} / \mathrm{dL})$ and a normal nucleated cell count $(1 / \mu \mathrm{L} ;$ reference range, $<5 \mu \mathrm{L}$ ) with normal cell cytology. A 2-week course of prednisone was prescribed (1mg/kg PO q12h for 1 week, then $0.5 \mathrm{mg} / \mathrm{kg} \mathrm{PO} \mathrm{q12h}$ for 1 week) while awaiting additional testing (urine metabolic scree, CSF culture, serum titers for Toxoplasma and Neospora). Clinical signs were not altered by prednisone treatment. Results of the urine metabolic screen were normal, CSF culture was negative for bacteria at 3 days, and serum IgG and IgM titers for Toxoplasma and Neospora were negative. Because of the progression of its neurologic signs, the dog was euthanized at approximately 26 months of age.

A fourth Australian Cattle Dog (Dog D) presented at the Animal Medical Center in New York City in 2015 at the age of approximately 24 months after a 6-month history of progressive behavior changes, including aggression toward people and 
other dogs, ataxia, anxiety, lethargy, and blindness. A neurologic examination identified the bilateral absence of the menace reflex and severely decreased nasal sensation. Pupillary light and palpebral reflexes were normal. The owners elected to have the dog euthanized immediately after the neurologic examination. Magnetic resonance imaging performed immediately after euthanasia showed diffuse symmetrical brain atrophy.

Australian Cattle dog E (Fig. 1D), a spayed female from Alabama, was euthanized in 2015 at approximately 27 months of age after having exhibited neurologic signs similar to the other 4 affected Australian Cattle dogs. Starting at about 6 months of age, the dog began to occasionally bump into obstacles, but continued to engage in vision-dependent activities, such as retrieving balls, without difficulty. Several months later, the dog began to exhibit signs of anxiety that became progressively more pronounced; it would become particularly agitated in response to loud noises. At approximately 2 years of age, the dog began to suffer from seizures and associated trance-like behavior, lost interest in activities it previously had enthusiastically engaged in, would pace compulsively, and began to suffer from progressive vision and coordination loss. Visual impairment was apparent in both dim and bright light. Upon neurologic examination at approximately 26 months of age, both direct and indirect pupillary responses could be elicited despite blindness. However, the dog did not exhibit a menace response on either side and physiologic nystagmus was observed in both eyes. Magnetic resonance imaging of the brain disclosed generalized brain atrophy, and enlarged ventricles. Cerebrospinal fluid analysis did not identify any abnormalities. Shortly after the 
neurologic examination, the dog's seizures became more frequent and the dog was euthanized. No tissue or blood samples were saved, but buccal swab samples were collected from both parents.

\section{Light and Electron Microscopic Procedures}

Cerebral cortical (parietal lobe) cerebellar and retinal samples were collected at necropsy from 4 of the affected Australian Cattle Dogs and examined by fluorescence and electron microscopy. The eyes from dogs A and B were enucleated and the corneas removed immediately. One eye from each dog was placed in a fixative consisting of 3.5\% formaldehyde, $0.05 \%$ glutaraldehyde, 120 $\mathrm{mM}$ sodium cacodylate, $1 \mathrm{mM} \mathrm{CaCl}$, and $\mathrm{pH} 7.4$ (immune fix), and the other eye was placed in $2.5 \%$ glutaraldehyde, $100 \mathrm{mM}$ sodium cacodylate, and pH 7.4 (EM fix). Slices of the cerebral cortex and cerebellum each were placed in the same fixatives. Before further processing, the eyecups were dissected to obtain regions from the posterior poles adjacent to the optic nerve heads and these regions were used for examination. All samples were incubated in these fixatives at room temperature until being further processed for microscopic examination. The same tissues were collected post-mortem from dogs $C$ and $D$ in a similar manner except that they initially were placed in $10 \%$ buffered formalin for shipment. In addition, a sample of heart ventricle wall was collected from dog $C$ in the same fixative. Upon receipt at the University of Missouri, the tissue samples were washed briefly in 170 $\mathrm{mM}$ sodium cacodylate, $\mathrm{pH} 7.4$ and then transferred to the same fixatives as used for the other dogs. These tissues were examined by previously described 
histopathologic, immunohistochemical, fluorescent microscopy, and electron microscopic procedures. ${ }^{143}$ Among these procedures was immunohistochemical staining of the cerebellar cortex and closely associated medulla were dissected from the brains fixed in immune fix (in some cases preceded by fixation in formalin). These tissues were embedded in paraffin and sectioned, the sections were immunostained for GFAP.

\section{Molecular Genetic Analysis}

EDTA-anticoagulated blood from affected Australian Cattle Dogs A, B, C and D described above served as a source of DNA. Buccal swab samples from the sire and dam of affected Australian Cattle Dog E were collected on commercial cards ${ }^{\mathrm{a}}$. Previously described procedures were used to isolate DNA from the blood ${ }^{117}$ and cards. ${ }^{156}$ In addition, DNA samples from 347 other Australian Cattle Dogs, 188 Border Collies, and 177 dogs representing 88 other breeds also were used in the study. These samples were randomly selected from among the samples in the University of Missouri Animal DNA repository.

The DNA from Australian Cattle Dog A was submitted to the University of Missouri DNA Core facility for the preparation of 2 PCR-free libraries (fragment sizes of approximately $350 \mathrm{bp}$ and $550 \mathrm{bp}$ ) with a commercial kit ${ }^{\mathrm{b}}$ and for $2 \times 100$ paired-

end sequencing in 2 flow-cell lanes in a massively parallel DNA sequencer. ${ }^{c}$ The adaptor sequences were trimmed with custom Perl scripts and the reads were error corrected using a previously described data analysis pipeline. ${ }^{117}$ These reads were deposited in the Sequence Read Archive (accession SRS834022) and 
aligned to the canine reference genome sequence (CanFam 3.1) using the default parameters of the bwa algorithm in BWA 0.7.12 software. ${ }^{157}$ Samtools 1.2 software was used to convert tab-delimited text files that contained sequence alignment data (aligned SAM file types) to binary versions (BAM file types), merge the libraries, and sort and index the files by genomic coordinates. ${ }^{158}$ The Platypus 0.8.1 variant caller software ${ }^{159}$ was used to generate individual variant call format (VCF) files using the default parameters. Commercial software ${ }^{d}$ was used to annotate variants and to compare them with similarly generated and annotated VCF files from 43 other control dogs with different genetic diseases. The same software ${ }^{d}$ was also used to filter the variants to identify those with the following 2 characteristics: (1) they were located within canine orthologs of the 13 genes associated with NCL in humans, ${ }^{137}$ and, (2) they were predicted to alter the primary structure of the gene product.

The DNA samples from the 4 affected Australian Cattle Dogs were genotyped by direct automated Sanger sequencing of PCR amplicons produced with oligonucleotide primers 5'-TTAACCAAATGGCAAAGTGGG-3' and 5'TTCTTGAACTCTGCTCCAAGT-3' which span a previously described casual CLN5:C.619C >T transition. ${ }^{142} \mathrm{~A}$ TaqMan allelic discrimination assay ${ }^{81}$ was used to genotype both parents of Australian Cattle Dog E and the archived DNA samples. The sequences of the PCR primers for the allelic discrimination assay were 5'GCGGGACAATGAAACAGGAATTTAT-3' and 5'-TGTCTCAGCCCCCTTTGTTG3'. The competing probes were 5'-CTGGCTTGAACAGTCC-MGB-3' (reference allele) and 5'-CTGGCTTAAACAGTCC-MGB-3' (variant allele). 


\section{Results}

\section{Light and Electron Microscopy}

All cerebella, cerebral cortical and retinal samples from the affected Australian Cattle Dogs exhibited abnormal accumulations of autofluorescent storage material with the fluorescence spectral characteristics typical of the NCLs (Fig. 3A-D). The cardiac muscle of a heart ventricle also was found to harbor substantial amounts of autofluorescent inclusions within the muscle fibers in the 1 affected dog from which the heart was examined (Fig. 3E). 

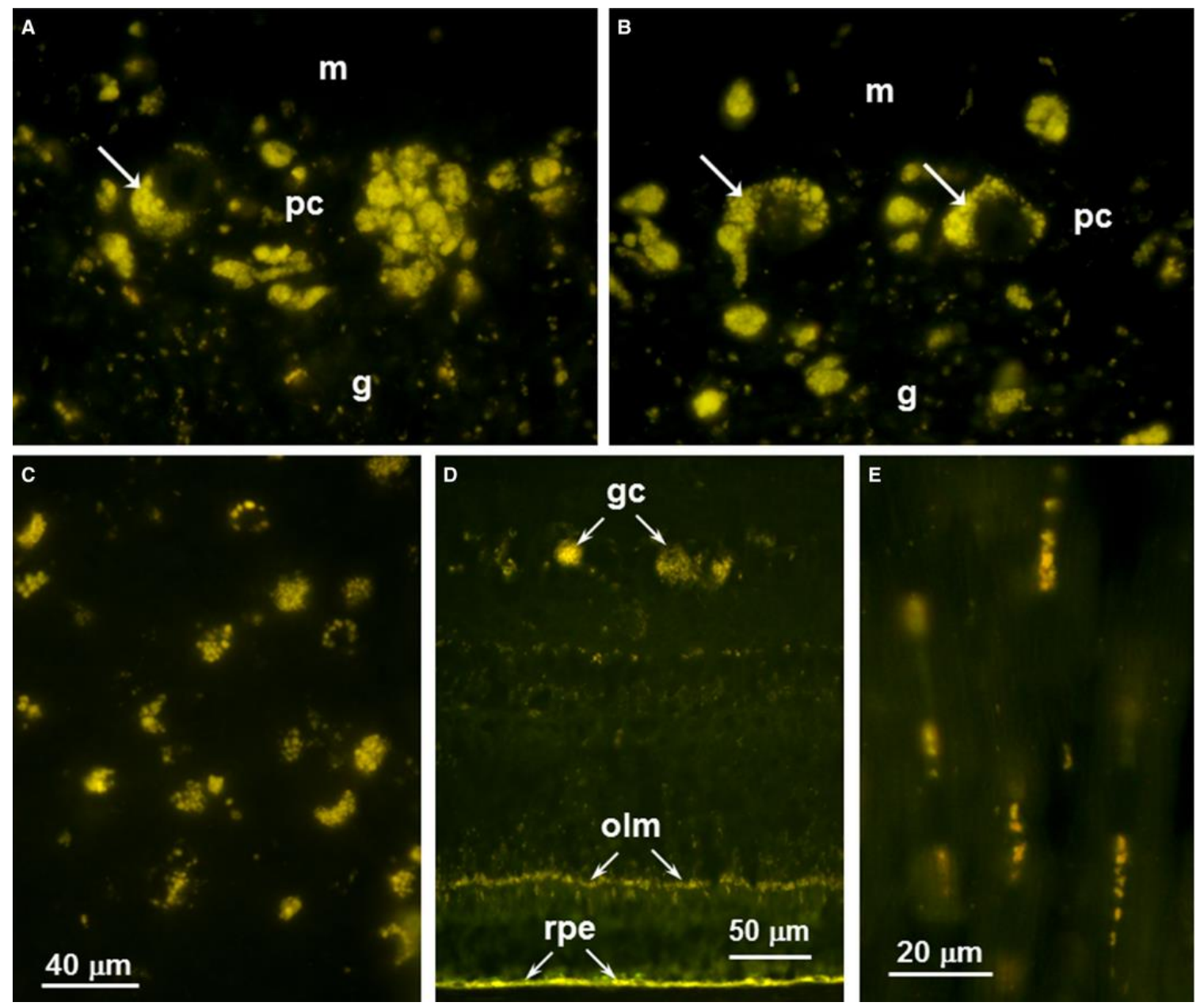

Fig. 3. Fluorescence micrographs of cryostat sections the cerebellum (A and $\mathbf{B}$ ), cerebral cortex (C), retina (D) from Dog A, and heart ventricular wall (E) from Dog C. In the cerebellum, storage body accumulation was most abundant in the Purkinje cell layer (pc) with some but not all of the material within cells that could be identified as Purkinje cells (arrows in $\mathbf{A}$ and $\mathbf{B}$ ). There were lesser accumulations of this material in the molecular $(\mathrm{m})$ and granular $(\mathrm{g})$ layers. In the cerebral cortex (B), the storage material was widely distributed throughout most of the tissue. In the retina (C), the most prominent accumulations of autofluorescent material were in the ganglion cells (gc) and along the outer limiting membrane (olm). The retinal pigment epithelium (rpe) also contained substantial amounts of material with similar fluorescence properties, but since the accumulation of such material occurs during normal aging, the presence of this material in the rpe is not diagnostic for NCL. Clusters of autofluorescent inclusions were present in the heart muscle fibers sections in longitudinal orientation (E).

The ultrastructural appearances of the storage material in brain and retina were

essentially the same for $\operatorname{dog} A$ and $B$ from which EM-fixed samples were obtained.

The contents of the storage bodies had a primarily membranous appearance in

cerebella Purkinje cells, cerebral cortical neurons, and retinal ganglion cells (Fig.

4). In all 3 cell types, the inclusions were membrane bound and consisted of 
aggregated clusters of membrane-like structures, but the arrangement of this membrane-like material varied among cell types. The primary storage components in the Purkinje cells had short linear and vesicular appearances (Fig. 4A). In the cerebral cortical neurons and retinal ganglion cells, storage bodies consisted almost exclusively of clusters of parallel stacks of relatively linear profiles (Fig. 4BD). The cerebral cortex contained storage bodies with scattered, extremely electron-dense structures (Fig. 4B). The degree of compaction of the membranelike structure in the retinal ganglion cell inclusions was variable (Fig $4 C$ and D). The storage bodies along the outer limiting membrane of the retina were located primarily within the photoreceptor cells just interior to the junctions between these cells and adjacent Mueller cells (Fig. 4E and F). These storage bodies contained either aggregates of small vesicles (Fig. 4E) or membrane-like material in a variety of configurations (Fig. 4F). The membrane-like components were randomly distributed within primarily granular material, unlike the clustered distributions found in the other cell types. The only cardiac tissue received from an affected dog was fixed in formalin, which did not permit good ultrastructural preservation of the storage material. 

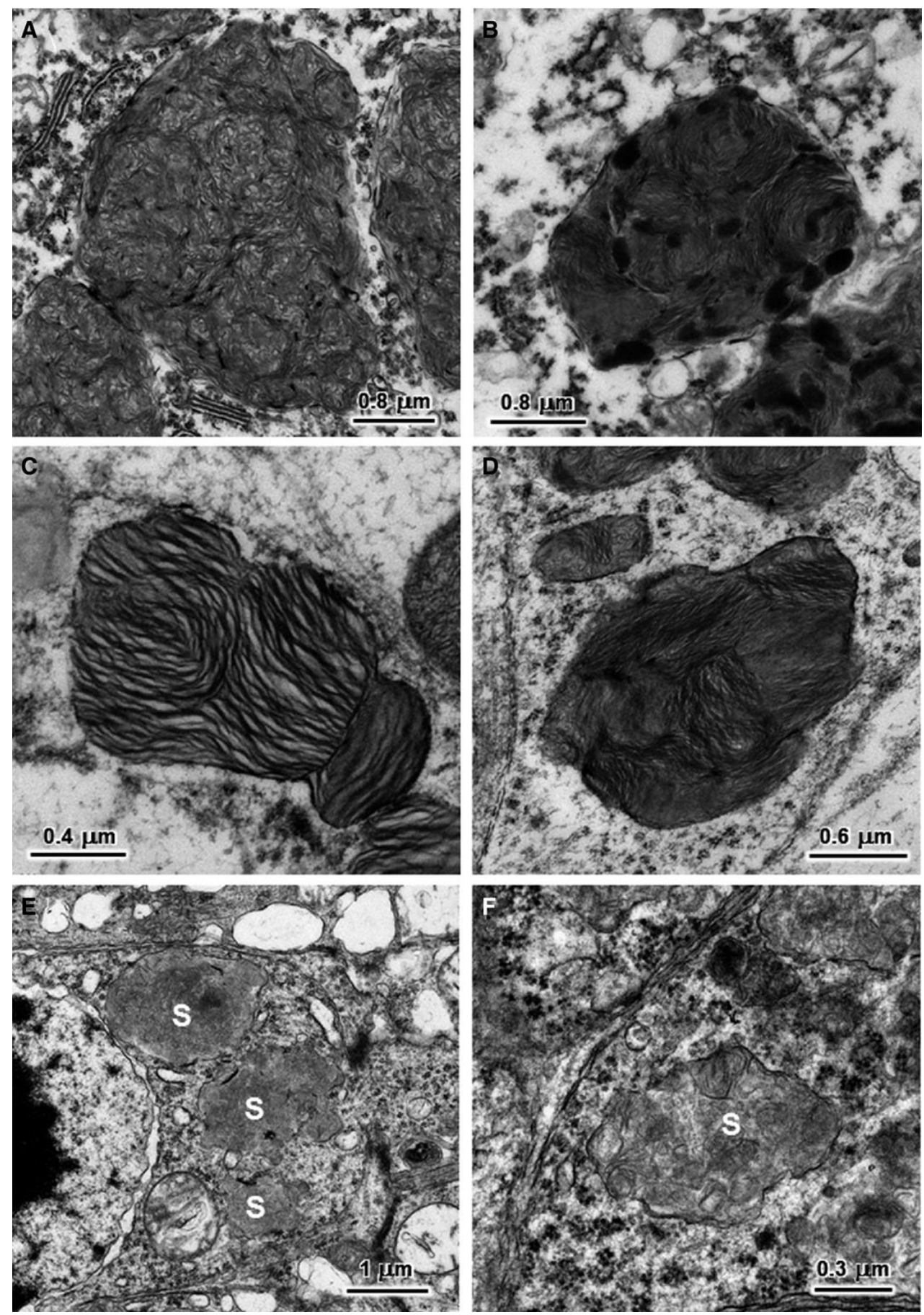

Fig. 4. Electron micrographs of the disease-related storage bodies from a cerebellar Purkinje cell (A), a cerebral cortical neuron (B), retinal ganglion cells (C and D), and retinal photoreceptor cells (E and F) of NCL-affected Australian Cattle Dog D. The storage bodies (s) in the photoreceptor cells are primarily located just internal to the outer limiting membrane. The contents of storage bodies consisted of both aggregates of small vesicles (E) or of membrane-like structures in a variety of configurations (F). 
The NCLs, like many other lysosomal storage diseases, are characterized by astrogliosis, as indicated by increased amounts of glial fibrillary acid protein (GFAP) in astrocytes. ${ }^{52,117,143,160}$ Examination of the cerebellar cortex from Australian Cattle Dog A by immunohistochemistry disclosed very high numbers of cells in both the cerebellar medulla and the granule cell layer that stained strongly with an anti-GFAP antibody (Fig. 5A and C). By comparison, little GFAP immunostaining was observed in either of these cerebellar areas in a normal healthy dog of similar age (Fig. 5B and D).
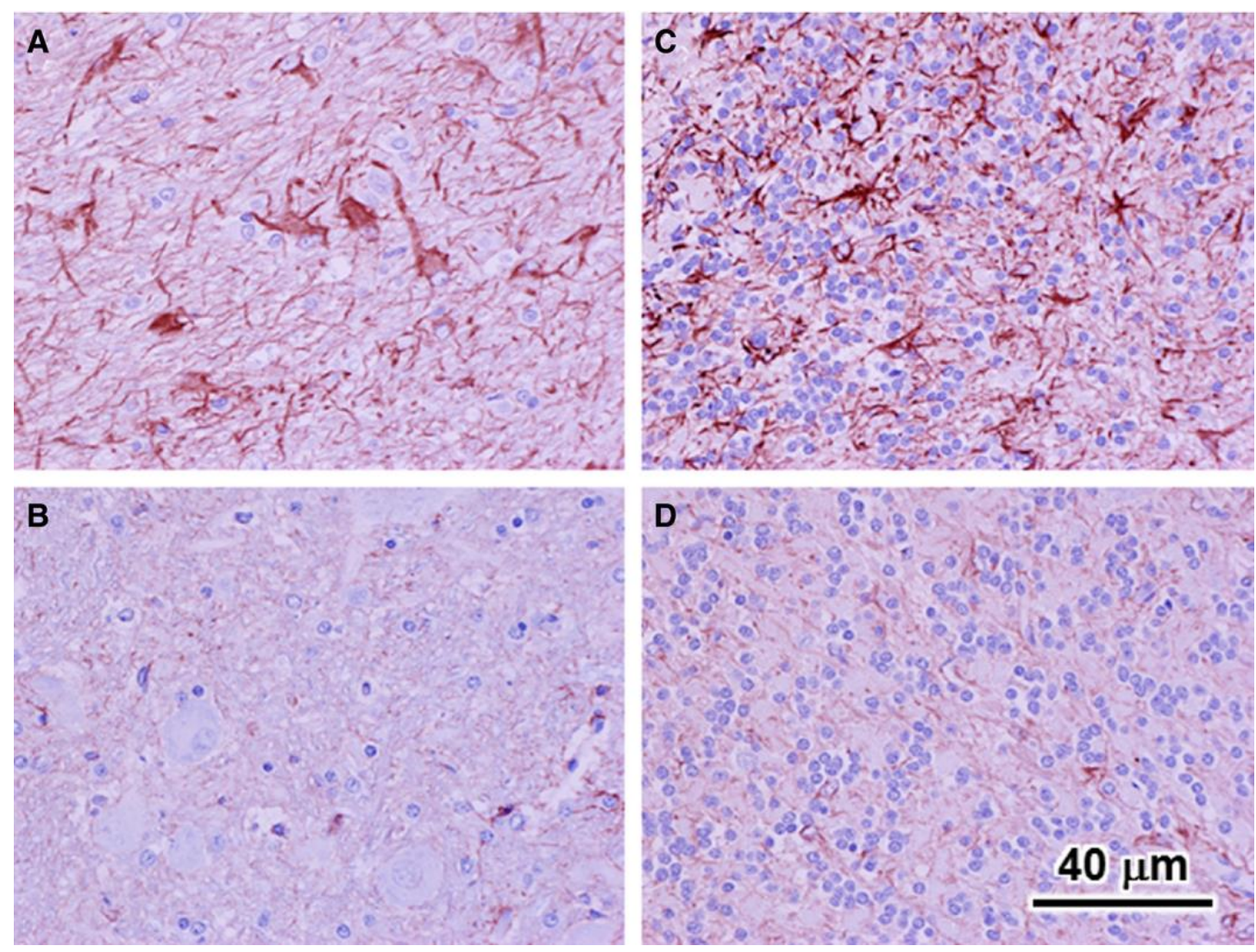

Fig. 5. Light micrographs of sections of the cerebellar medulla ( $\mathbf{A}$ and $\mathbf{B})$ and granule cell layer (B and D). All sections were immunostained for GFAP. GFAP staining is a reddish brown color. All photomicrographs are from the cerebellum. Micrographs $(\mathbf{A})$ and $(\mathbf{C})$ are from the affected Australian Cattle Dog A; micrographs (B) and (D) are from a normal healthy Beagle of similar age. Bar in (D) indicates magnification of all 4 micrographs. GFAP-positive cells with the typical profiles of reactive astrocytes were abundant in both the granule cell layer and medulla of affected dog, but were not present in either of these areas of the cerebellar cortex from the normal dog. 


\section{Molecular Genetics}

We used massively parallel sequencing technology to generate a whole-genome sequence from NCL-affected Australian Cattle Dog A and aligned the sequence reads to the canine genome reference sequence (CanFam3.1). The average depth of coverage of the aligned sequence was 28 -fold. The 6.2 million potential sequence variants detected in this alignment were filtered to retain only those that were predicted to alter the amino acid sequences of any of the canine orthologs of the 13 genes known to have harbored mutations in human NCL patients. ${ }^{137}$ As shown in Table 2, only 5 sequence variants survived this filtration process. Four were missense mutations that occurred in heterozygous state. These 4 variants were also present in from 5 to 13 of the 43 control whole-genome sequences from dogs with no signs of NCL and, thus, unlikely to have been responsible for Australian Cattle Dog A's NCL. In contrast, a $C$ to $T$ transition at position 30,574,637 bp on CFA22 was homozygous in Australian Cattle Dog A and produced a premature stop codon that was predicted to severely truncate the encoded CLN5. This variant was almost certainly causal because it previously had been reported to be responsible for NCL in Border Collies. ${ }^{142}$ The homozygous CLN5:C.619C>T transition, detected in the whole-genome sequence from Australian Cattle Dog A, was confirmed by automated Sanger sequencing. In addition, automated Sanger sequencing showed that Australian Cattle Dog B, C and $\mathrm{D}$ also were homozygous for the CLN5:C.619T allele. 
Table 2. Variants in NCL-associated genes in WGS from affected Australian Cattle Dog A.

\begin{tabular}{|c|c|c|c|c|c|}
\hline $\begin{array}{l}\text { Chromosomal } \\
\text { Coordinates }\end{array}$ & Gene & $\begin{array}{l}\text { Nucleotide } \\
\text { Change }\end{array}$ & $\begin{array}{c}\text { Predicted } \\
\text { Amino Acid } \\
\text { Change }\end{array}$ & Zygosity & Comments \\
\hline $6: 18257856$ & CLN3 & c. $209 A>G$ & p.Glu70Gly & Heterozygous & $\begin{array}{l}\text { Variant in } 10 \\
\text { other WGSs }\end{array}$ \\
\hline 18:46011691 & CTSD & c.1168T>C & p.Cys390Arg & Heterozygous & $\begin{array}{l}\text { Variant in } 10 \\
\text { other WGSs }\end{array}$ \\
\hline 18:46011691 & CTSD & c. $761 A>C$ & p.Lys254Thr & Heterozygous & $\begin{array}{l}\text { Variant in } 13 \\
\text { other WGSs }\end{array}$ \\
\hline 21:2992271 & TPP1 & $c .1280 C>T$ & p.Ala427Val & Heterozygous & $\begin{array}{c}\text { Variant in } 5 \text { other } \\
\text { WGSs }\end{array}$ \\
\hline 22:30574637 & CLN5 & c. $619 C>T$ & p.GIn207* & Homozygous & $\begin{array}{c}\text { Unique to } \\
\text { affected dog } \\
\text { WGS }\end{array}$ \\
\hline
\end{tabular}

A TaqMan allelic discrimination assay ${ }^{81}$ was used to determine the CLN5:C.619 genotypes for another 714 dogs, including both parents of the affected Australian Cattle Dog E. These 2 parents and 1 other Australian Cattle Dog represented in our DNA collection since 2014, were CLN5:c.619C/T heterozygotes. The variant c.619T allele was not detected in archived DNA samples from 346 other Australian Cattle Dogs previously obtained for a variety of reasons unrelated to NCL. In addition, all 188 randomly selected Border Collie DNA samples and all 177 randomly selected samples from representatives of 88 other breeds were homozygous for the reference c. $619 \mathrm{C}$ allele. 


\section{Discussion}

We used fluorescence and electron microscopy to confirm the diagnosis of NCL in 4 apparently unrelated Australian Cattle Dogs that had exhibited neurologic signs consistent with this disorder. Previously, NCL had been diagnosed in 2 pairs of Australian Cattle Dog littermates over 35 years ago. ${ }^{149,161,162}$ Both of the previously reported litters were from the United States, but there was no known familial relationship between them. ${ }^{161}$ Both sexes were affected. One litter included an affected pair and 7 clinical normal littermates. ${ }^{149}$ The clinical status of the littermates to the other affected pair was not reported, but it was reported that their parents were normal. ${ }^{161}$ These observations are consistent with autosomal recessive mode of inheritance. The earlier-reported NCL-affected Australian Cattle Dogs and those that we studied had brains with similar distributions of autofluorescent storage bodies with similar ultrastructural appearance. In addition, the ages at onset of euthanasia of these earlier-reported Australian Cattle dogs with NCL are similar to those of the NCL-affected Australian Cattle Dogs reported here (Table 3). Thus, it is likely that a single founding mutation was responsible for both the earlier-reported Australian Cattle Dog NCL and for the affected Australian Cattle dogs reported here. Nonetheless, biological samples from the earlierreported dogs were not available for testing and the existence of NCL phenocopies within the Australian Cattle Dog breed cannot be excluded.

Whole-genome sequencing has proven to be an efficient strategy for the identification of the mutations responsible for NCL in dogs. ${ }^{51,52,117,143}$ Thus, in search of the molecular cause of NCL in Australian Cattle Dogs, we generated a 
whole-genome sequence with DNA from affected Australian Cattle Dog A and identified a homozygous nonsense mutation, CLN5:c.619C>T, that had already been reported to cause NCL in Border Collies. ${ }^{142}$ The NCL-affected Australian Cattle Dogs, B, C and D also were homozygous for the variant c.619T allele, whereas there were no c.619T homozygotes among 349 other Australian Cattle Dogs not known to have exhibited clinical signs of NCL. Three of those 349 Australian Cattle Dogs were c.619C/T heterozygotes including both parents of Australian Cattle Dog E. The similarities between the clinical history of Dog E and the clinical histories of the other $4 \mathrm{NCL}$-affected Australian Cattle Dogs, and the fact that both of Dog's E parents carried the rare c.619T allele strongly imply that Dog E was a c.619T homozygote. The association of NCL in 2 dog breeds with the same rare truncating mutation in a candidate gene strongly indicates that the mutation is causal for NCL in both Australian Cattle Dog and Border Collies.

Although there are anecdotal accounts of "dog show" Border Collies with NCL-like signs in Australia and New Zealand in the 1970 s, ${ }^{163}$ the first published report of NCL in Border Collies appeared in $1988 .{ }^{164}$ This report described the disease in 3 male and 2 female Border Collies. Three years later, the clinical signs and laboratory findings for these 5 dogs and 18 other Border Collies were summarized. ${ }^{165}$ As shown in Table 3, the age at disease onset and the disease duration in these Border Collies from Australia closely resembled that of the affected Australian Cattle Dogs, including those shown here to be CLN5:c.619C>T homozygotes. Subsequently, a Border Collie from the United States was reported to have NCL with similar clinical and laboratory findings but a later age at onset. ${ }^{166}$ 
Table 3.Comparison of ages at onset and death or euthanasia for Australian Cattle Dogs, Border Collies, and Golden Retrievers with NCL.

\begin{tabular}{|c|c|c|c|c|c|c|}
\hline Breed & $\begin{array}{l}\text { Number } \\
\text { of dogs }\end{array}$ & Causal Mutation & Location & $\begin{array}{l}\text { Age at } \\
\text { Onset }\end{array}$ & $\begin{array}{l}\text { Age at Death } \\
\text { or } \\
\text { Euthanasia }\end{array}$ & Reference \\
\hline $\begin{array}{l}\text { Australian } \\
\text { Cattle Dog }\end{array}$ & 4 & CLN5:C.619T & U.S.A. & $\begin{array}{l}12 \text { to } 19 \\
\text { months }\end{array}$ & $\begin{array}{l}15 \text { to } 26 \\
\text { months }\end{array}$ & $\begin{array}{l}\text { Current } \\
\text { report }\end{array}$ \\
\hline $\begin{array}{l}\text { Australian } \\
\text { Cattle Dog }\end{array}$ & 4 & Unknown & U.S.A. & $\begin{array}{l}12 \text { to } 14 \\
\text { months }\end{array}$ & $\begin{array}{l}18 \text { to } 26 \\
\text { months }\end{array}$ & 149,162 \\
\hline $\begin{array}{l}\text { Border } \\
\text { Collie }\end{array}$ & 23 & Unknown & Australia & $\begin{array}{l}16 \text { to } 23 \\
\text { months }\end{array}$ & $\begin{array}{c}18 \text { to } \\
29 \text { months }\end{array}$ & 165 \\
\hline $\begin{array}{l}\text { Border } \\
\text { Collie }\end{array}$ & 1 & Unknown & U.S.A. & $\begin{array}{c}27 \\
\text { months }\end{array}$ & 29 months & 166 \\
\hline $\begin{array}{l}\text { Border } \\
\text { Collie }\end{array}$ & 27 & CLN5:C.619T & Japan & $\begin{array}{l}15 \text { to } 20 \\
\text { months }\end{array}$ & $\begin{array}{l}23 \text { to } 32 \\
\text { months }\end{array}$ & 167 \\
\hline $\begin{array}{c}\text { Golden } \\
\text { Retriever }\end{array}$ & 4 & CLN5:c.934_935delAG & U.S.A. & $\begin{array}{l}13 \text { to } 17 \\
\text { months }\end{array}$ & $\begin{array}{c}30 \text { to } \\
34 \text { months }\end{array}$ & 143 \\
\hline
\end{tabular}

In 2005, linkage analysis with DNA markers from candidate regions placed the Border Collie NCL locus on a CLN5-containing segment of canine chromosome 22 and the CLN5:C.619C>T transition was identified as the causal mutation. ${ }^{142}$ Since then, it has been possible to use DNA tests to identify c.619T homozygotes and to investigate the Border Collie NCL with genetically defined cohorts of NCLaffected dogs. Two reports described CLN5:C619T homozygous Border Collies from Japan. ${ }^{167,168}$ The clinical laboratory findings for 27 Border Collies ${ }^{167}$ with genetically defined NCL were similar to those from earlier reports of affected Border Collies and Australian Cattle Dogs and to those of the 5 Australian Cattle Dogs in our report. The ages at onset and death also were similar in each group of affected dogs (Table 3). The Japanese investigators found a CLN5:c.619T allele frequency $>0.3$ in dogs from certain kennels that unknowingly included asymptomatic heterozygotes in their breeding stock ${ }^{167}$ and estimated an overall 
CLN5:C.619T allele frequency of 0.04 , based on random survey of over 400 Japanese Border Collies. ${ }^{168}$ The mutant $\mathrm{T}$ allele appears to be less common in North America because 188 Border Collie samples from our collection all were CLN5:C.619C allele homozygotes. Although possible, it is very unlikely that the CLN5:C.619C>T transition arose independently in Border Collies and Australian Cattle Dogs. Both breeds are of similar size and both breed are used for herding. Some kennels produce puppies from both dog breeds. Thus, there is the potential for occasional planned or inadvertent exchange of genetic material between breeds. Published reports indicate that the disease has been present in both breeds for many generations, ${ }^{149,164}$ but it is not possible from the available evidence to identify the breed of origin. Regardless of the origin, a DNA test for the mutation can be used to aid in the diagnosis of NCL.

A different CLN5 mutation, CLN5:c.934_935delAG, was found in the homozygous state in a Golden Retriever with NCL. ${ }^{143}$ This mutation produces a reading frameshift and is predicted to encode a truncated gene product: CLN5:pE312Vfs ${ }^{*} 6$. As shown in Table 3, the disease characteristics of the affected Golden Retrievers are similar to those of the affected Australian Cattle Dogs and Border Collies.

Mutations in human CLN5 were first reported to cause NCL in $1998 .{ }^{169}$ Since then, at least 36 potentially causal CLN5 sequence variants have been found in human NCL patients (http://www.ucl.ac.uk/ncl/cln5.shtml). For most CLN5 patients, the onset of clinical signs occurred between 4 and 7 years of age, ${ }^{170,171}$ but some presumably hypomorphic mutations have produced much later disease onsets. ${ }^{172}$ 
Although CLN5 mutations have been recognized as causes of NCL for over 16 years, the biological functions of the gene product and the mechanism leading to the neurodegeneration remain unknown. ${ }^{173}$ Ovine and murine Cln5-deficient animal models share disease features with human CLN5 patients ${ }^{174,175}$ and have been used to investigate the underlying disease pathways. ${ }^{176-178}$ The ovine model is involved in ongoing and planned experiments to evaluate potential therapies for human CLN5 patients. ${ }^{179}$ Several canine models have been used to develop therapies for lysosomal storage diseases, ${ }^{180}$ and dogs have obvious advantages over sheep in urban research settings. A TPP1-deficient canine NCL model ${ }^{141}$ has proven useful in the evaluation of therapies for the human CLN2 form of NCL including an enzyme replacement therapy that is currently in clinical trials in human patients (https://clinicaltrials.gov/ct2/show/NCT01907087). ${ }^{181-183}$ Dogs with CLN5 deficiency have the potential for similar use.

\section{Footnotes}

${ }^{1}$ Whatman FTA Elute card, catalog number WB120411, Fisher Scientific, Chicago IL

${ }^{2}$ TruSeq DNA PCR-Free Sample Preparation Kit, Illumina, San Diego, CA ${ }^{3}$ HiSeq 2500 System, Illumina, San Diego, CA

${ }^{4}$ SNP \& Variation Suite v8.x, Golden Helix, Inc., Bozeman, MT 


\section{Chapter 4}

\section{A homozygous PPT1 splice donor mutation in a Cane Corso Dog with neuronal ceroid lipofuscinosis}

\section{Abstract}

A 10-month-old spayed female Cane Corso dog was evaluated after a 2-month history of progressive blindness, ataxia and lethargy. Neurologic examination abnormalities indicated a multifocal lesion with primarily cerebral and cerebellar signs. Clinical worsening resulted in humane euthanasia. On necropsy there was marked gliosis throughout white matter tracts of the cerebrum, most prominently in the corpus callosum with moderate numbers of swollen axons and occasional spheroids. In the cerebral cortex and midbrain most neurons contained large amounts of autofluorescent storage material in the perinuclear area of the cells. Cerebellar storage material was present in the Purkinje cells, granular cell layer, and perinuclear regions of neurons in the deep nuclei. Neuronal ceroid lipofuscinosis (NCL) was diagnosed. Whole genome sequencing identified a PPT1c.124+1G>A splice donor mutation. This non-reference assembly allele was homozygous in the affected dog, has not previously been reported in dbSNP, and was absent from the whole genome sequences of 45 control canines and 31 unaffected Cane Corsos. Our findings indicate a novel mutation causing the CLN1 form of NCL in a previously unreported dog breed. A canine model for CLN1 disease could provide an opportunity for therapeutic advancement, benefiting both humans and canines with this disorder. 


\section{Case Report}

A 10-month-old, $29 \mathrm{~kg}$ (63 lb), spayed female Cane Corso dog was referred to the University of Wisconsin, School of Veterinary Medicine with a 2-month history of progressive blindness, ataxia and lethargy. The dog was acquired by a family from a breeder at 8 weeks of age and was the only dog in the household. Both parents of the dog were healthy at the time this case presented. We were unable to obtain information on the health status of any littermates. Approximately 2 months prior to presentation, the dog had difficulty navigating in low light. Over the next 2 months, the visual impairment progressed to apparent blindness. Progressive ataxia was noted starting approximately 1 month prior to presentation, prompting referral.

On initial evaluation at the University of Wisconsin, the dog's physical examination was within normal limits. Abnormalities identified during a complete neurologic examination included a mild right head tilt, a positional right multifocal, most apparent in the prosencephalon and cerebellum. The preliminary differential diagnoses were meningoencephalitis, hydrocephalus, abiotrophy, neoplasia or lysosomal storage disorder.

The recommended diagnostic tests included a complete blood count, blood chemistry, urinalysis, urine metabolic screen for inborn ventral strabismus, an absent menace response bilaterally with intact pupillary light reflexes elicited by bright light stimuli, an absent paw replacement test of the left pelvic limb, and a moderate vestibular ataxia with truncal sway and hypermetria of all limbs. The 
neuroanatomic lesion localization was considered errors of metabolism, infectious disease testing, thoracic radiographs, brain magnetic resonance imaging and cerebrospinal fluid analysis. All testing was declined except the urine metabolic test and infectious disease testing. Serum distemper by RT-PCR, serum Neospora caninum by IFA, the Cryptococcus neoformans antigen test and Blastomycosis urine antigen were all negative. The urine metabolic screen ${ }^{\mathrm{a}}$ showed a slightly positive mucopolysaccharidosis spot test, which was attributed to young age, and glutamine and taurine levels were slightly above the reference range. The dog was discharged with instructions to administer prednisone ( $0.5 \mathrm{mg} / \mathrm{kg}$ per os $\mathrm{q} 12 \mathrm{~h})$. Because the neurological condition continued to deteriorate, euthanasia was elected 5 days after discharge.

A complete necropsy was performed. Abnormal findings were limited to the central nervous system. The brain was bilaterally symmetrical and the cerebellar size was proportionate to the cerebrum, with no evidence of cerebellar herniation or ventricular dilatation. Histologic abnormalities were found in the cerebrum and cerebellum. In the cerebrum, there was marked gliosis throughout white matter tracts, most prominently in the corpus callosum. The affected dog exhibited a dramatically lower cell density in the external granular layer of the cerebral cortex compared to an age-matched normal Beagle (Fig. 1 A and B). In the affected dog, this brain layer also exhibited pronounced GFAP labeling of the astrocytes that was not observed in the brain from the normal dog (Fig.1 C and D). Substantial disease-related GFAP immunostaining was also observed in the cerebral cortical white matter tracts of the affected dog. Cerebral cortical neurons of the affected 
dog also contained large aggregates of PAS-positive material in the perinuclear cell bodies (Fig. 2). The granular layer of the cerebellar folia was markedly hypocellular, and marked astrogliosis was noted throughout all layers of the cerebellar cortex and in the arbor vitae white matter as indicated by immunohistochemical staining for GFAP (Fig. 3). The retinas were not adequately prepared to evaluate for truncation of the photoreceptor outer segments as reported in a human case of PPT1 deficiency, ${ }^{184}$ but dark-staining inclusions could be seen surrounding the nuclei in some retinal cells.

Based on the clinical and histological findings, neuronal ceroid lipofuscinosis (NCL) was included among the differential diagnoses. Thus, paraffin blocks, formalinfixed tissues and EDTA-anticoagulated blood were shipped to the University of Missouri for further analysis. The NCLs are lysosomal storage diseases in which autofluorescent storage inclusions with distinct ultrastructure accumulate in the cytoplasm of neurons and other cell types. The NCLs are almost always recessively inherited and affected individuals experience progressive neurodegeneration and premature death. ${ }^{171}$ 

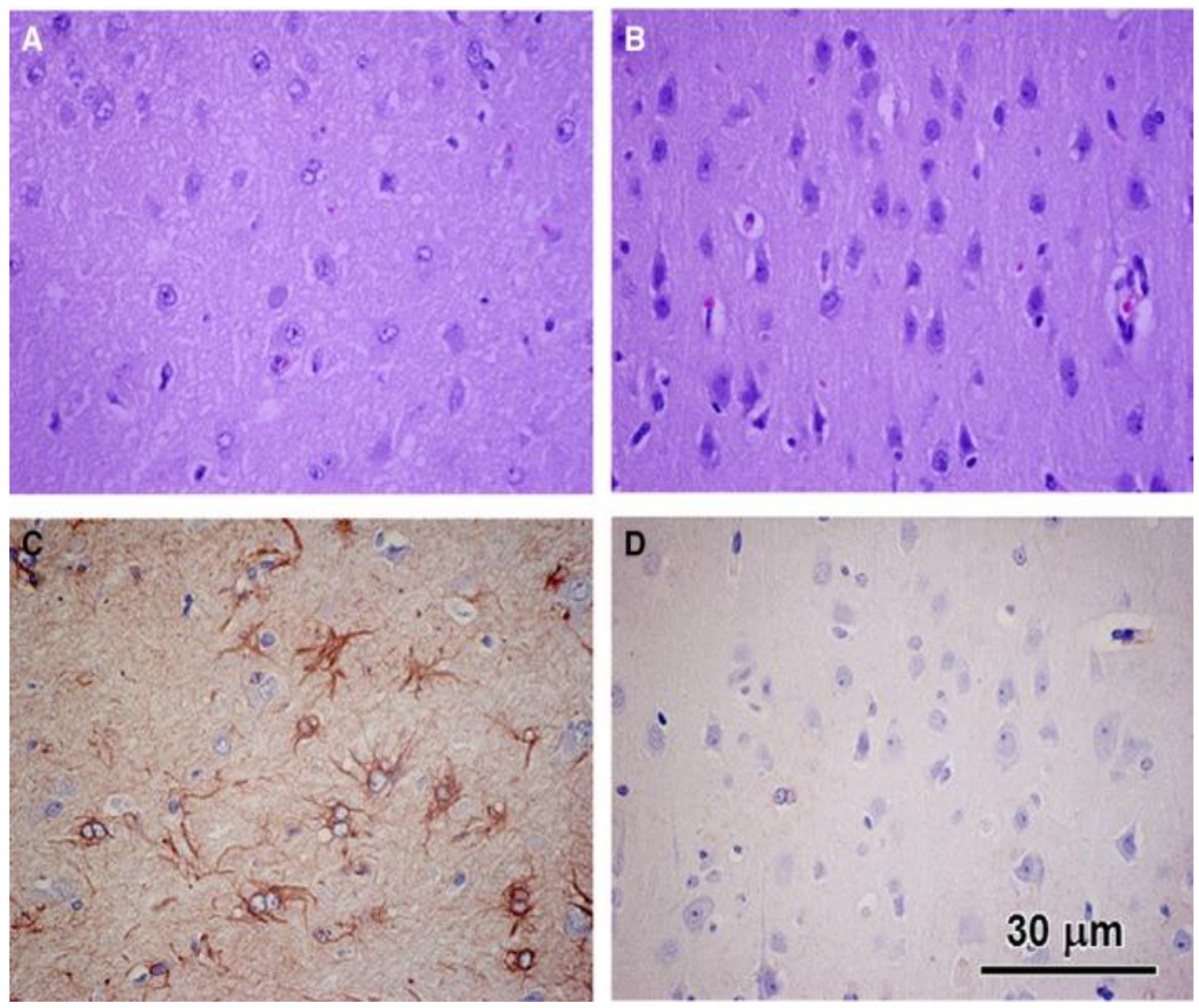

Fig. 1. Light micrographs of paraffin sections of the cerebral cortex external granular layer from the neuronal ceroid lipofuscinosis-affected Cane Corso ( $\mathbf{A}$ and $\mathbf{C}$ ) and from an age-matched normal Beagle (B and D). A and $\mathbf{B}$ were stained with hematoxylin and eosin. $\mathbf{C}$ and $\mathbf{D}$ were immunostained with an anti-GFAP antibody. The diseased dog exhibited a substantial reduction in cell density in the external granular layer and substantial numbers of GFAP-labeled astrocytes that were not observed in the control dog sample. Bar in (D) indicates magnification of all micrographs. 


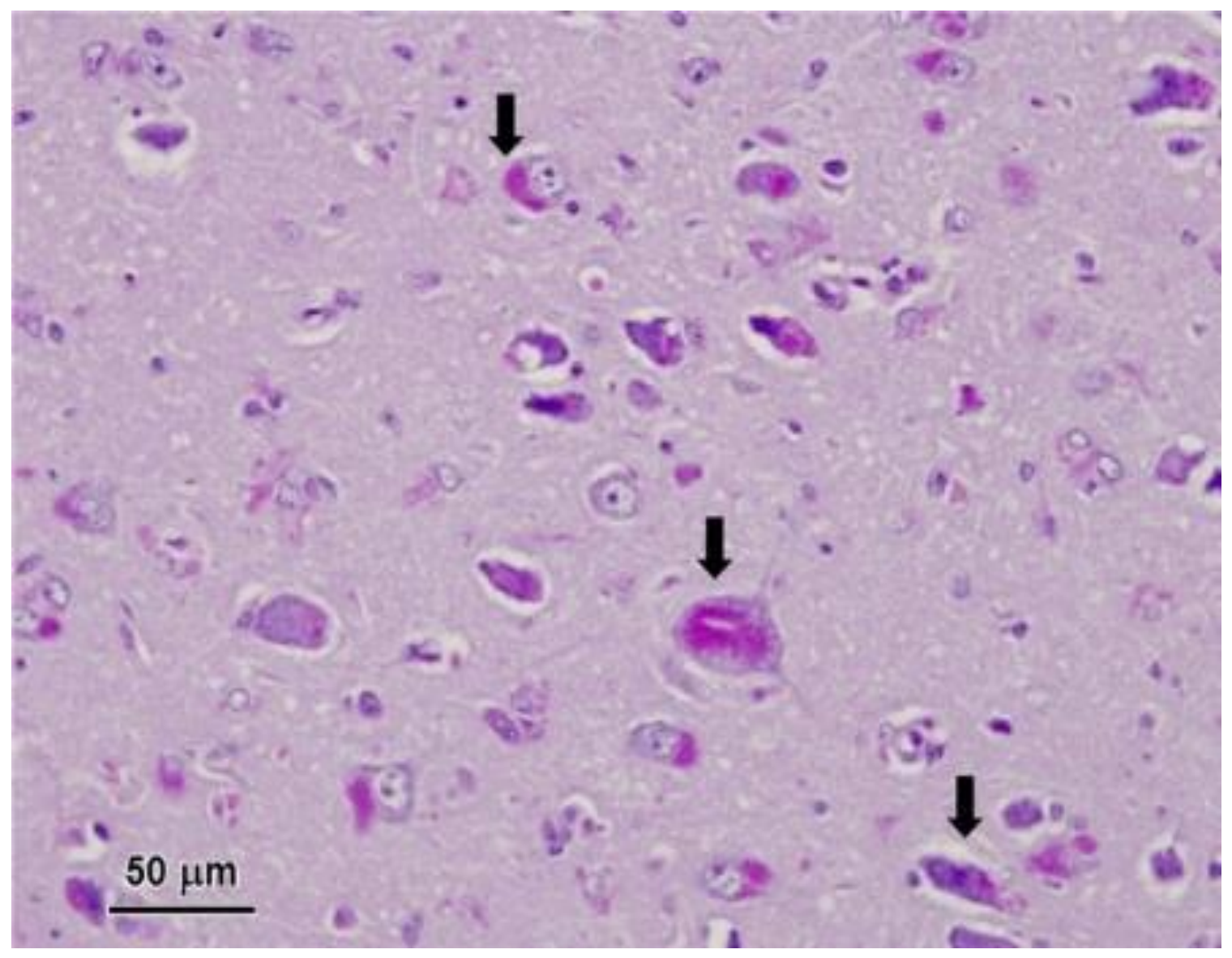

Fig. 2. Light micrographs of diastase-treated PAS-stained section of the cerebral cortex from the affected dog. Aggregates of PAS-staining inclusions were present in the perinuclear areas of most cortical neurons (arrows). 

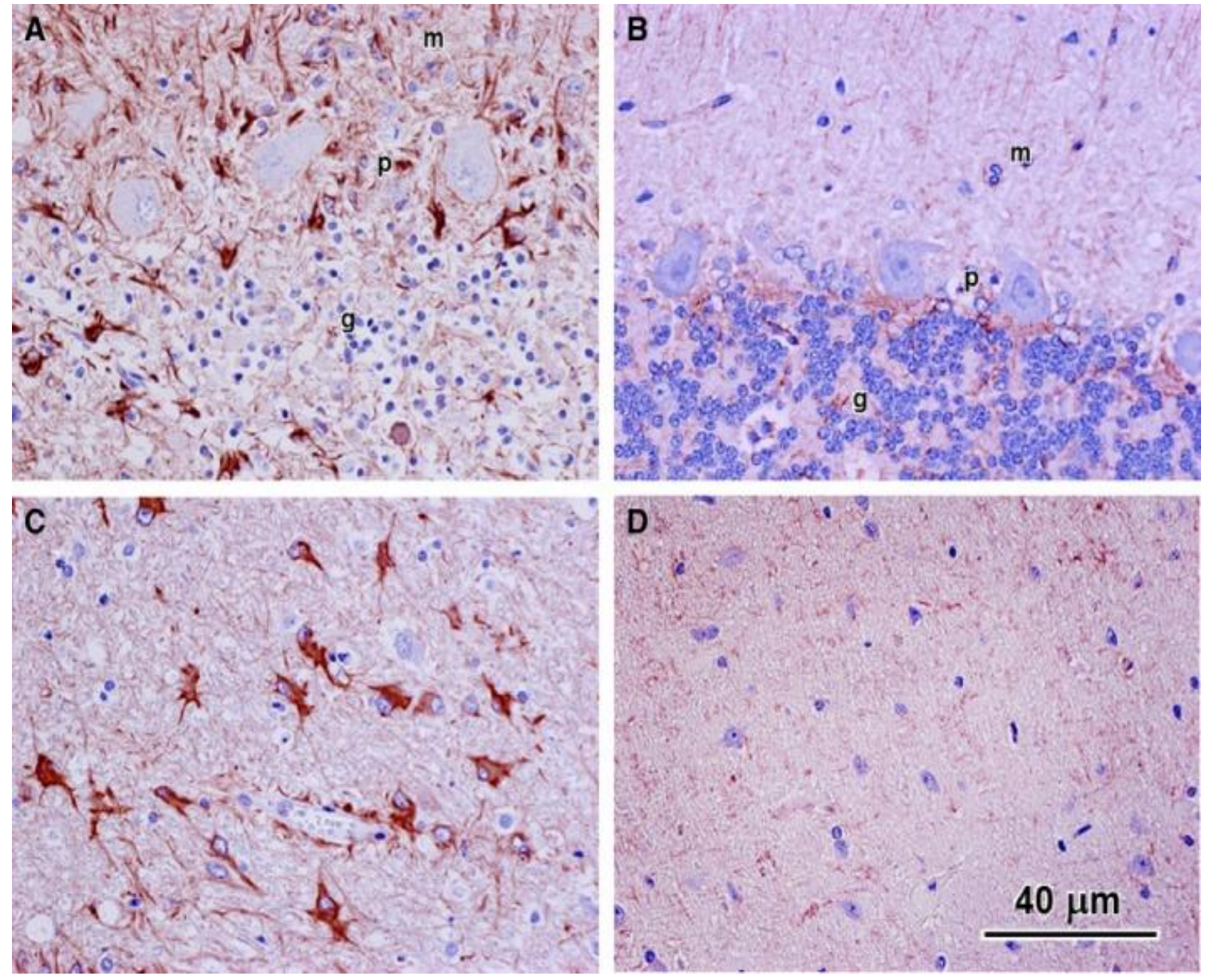

Fig. 3. GFAP immunohistochemical stained sections of the cerebellum from the affected Cane Corso ( $\mathbf{A}$ and $\mathbf{C}$ ) and from a normal Beagle of similar age (B and $\mathbf{D})$. Images $(\mathbf{A})$ and $(\mathbf{B})$ show regions of the cerebellar cortex and images $(\mathbf{C})$ and $(\mathbf{D})$ show regions of the arbor vitae at the bases of the folia. In the affected dog, cells showing pronounced GFAP immunostaining were present in all layers of the cortex ( $\mathrm{m}$ : molecular layer, $\mathrm{p}$ : Purkinje cell layer; g: granular cell layer) (A) as well as in the arbor vitae (B). No cells exhibited substantial GFAP immunostaining in either the cerebellar cortex or arbor vitae of the normal dog (B and $\mathbf{D})$. Note also the sparsity of cell nuclei in the granular cell layer of the affected dog $(\mathbf{A})$ compared to the normal dog $(\mathbf{B})$.

Previously described fluorescent microscopic procedures ${ }^{143}$ were used to examine

the tissues from the affected Cane Corso. Massive accumulations of autofluorescent storage material were present throughout the brain and the retina (Fig. 4). In the cerebral cortex and midbrain almost all of the neurons contained large amounts of this material concentrated primarily in the perinuclear areas of the cells (Fig. 4A and B). In the cerebellar cortex the storage material was present 
in the Purkinje cells and in large masses in the granular cell layer (Fig. 4C). Large amounts of the autofluorescent storage material were also present in the perinuclear regions of neurons in the deep cerebellar nuclei (Fig. 4D). In the retina the storage material was concentrated primarily in the ganglion cells, although small amounts of the autofluorescent material were scattered throughout the retina with a relatively large amount forming a continuous layer along the outer limiting membrane (Fig. 4E).

Small pieces of formalin-fixed cerebellar and cerebral cortex and retina were washed in sodium cacodylate buffer and then incubated in cacodylate-buffered $2.5 \%$ glutaraldehyde before being processed for electron microscopic examination. In the Purkinje cells, cerebral cortical neurons and retinal ganglion cells, the storage body contents appeared to consist primarily of aggregates of membrane-like structures. In the Purkinje cells these structures were similar in appearance to mitochondrial cristae (Fig. 5A), whereas, in the other cell types the material was much more condensed with the membrane-like profiles tightly packed together (Fig. 5B and $\mathrm{C}$ ). Although the aggregated material formed distinct structures in all 3 tissues, membranes enclosing these aggregates were not observed except in the case of some Purkinje cell inclusions, most likely because such membranes are not well preserved with formalin fixation. 

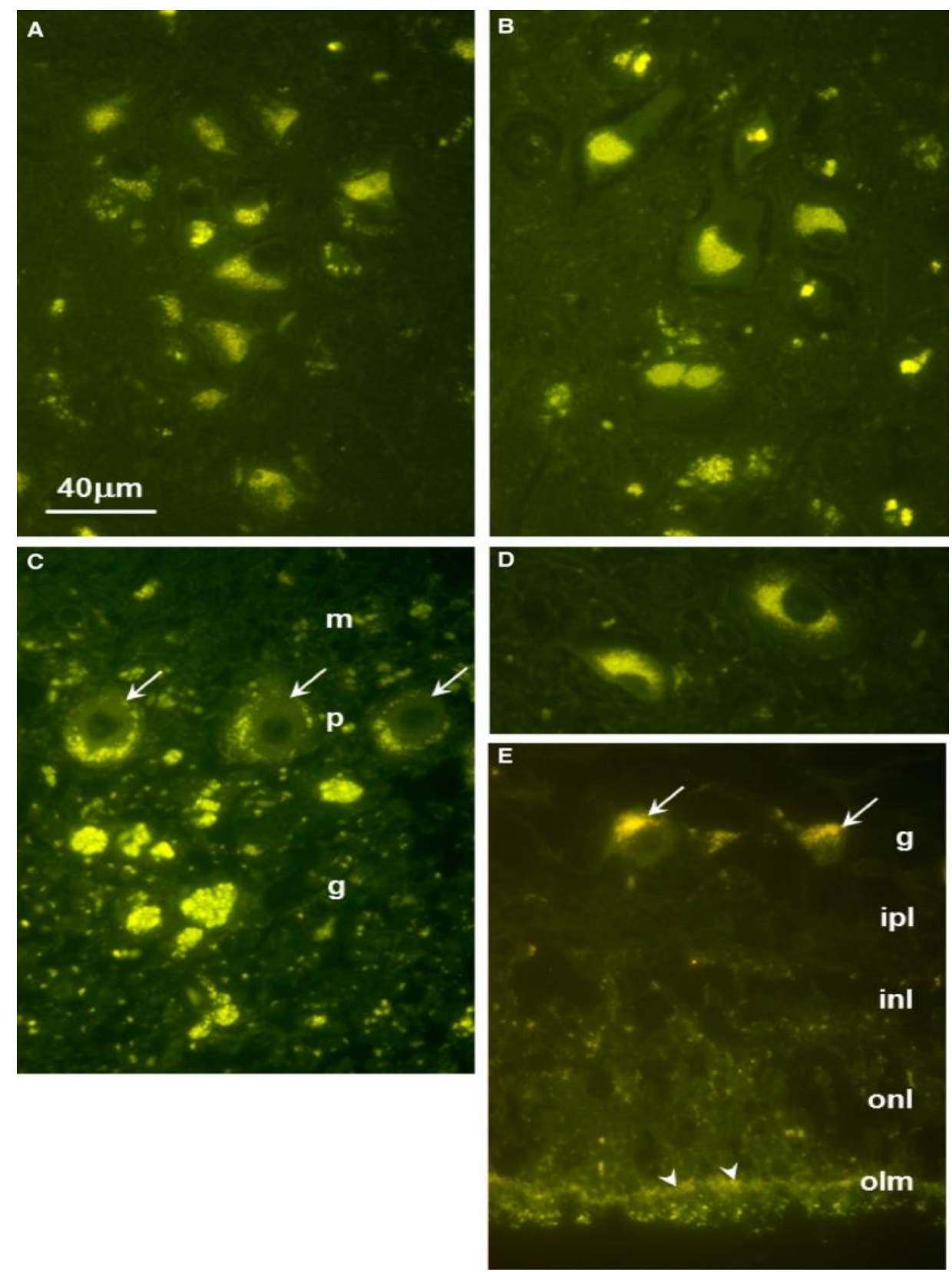

Fig. 4. Fluorescence micrographs of unstained sections of $(\mathbf{A})$ cerebellar cortex, $(\mathbf{B})$ cerebral cortex, (C) retina, (D) deep cerebellar nucleus, and (E) retina. In (A), arrows point to Purkinje cells; m: molecular layer, p: Purkinje cell layer, g: granular cell layer. In (C), arrows point to Purkinje cell bodies. In (E), arrows point to retinal ganglion cells and arrow heads point to the outer limiting membrane; g: ganglion cell layer, ipl: inner plexiform layer, inl: inner nuclear layer, onl: outer nuclear layer. Bar in (A) indicates magnification of all micrographs. 

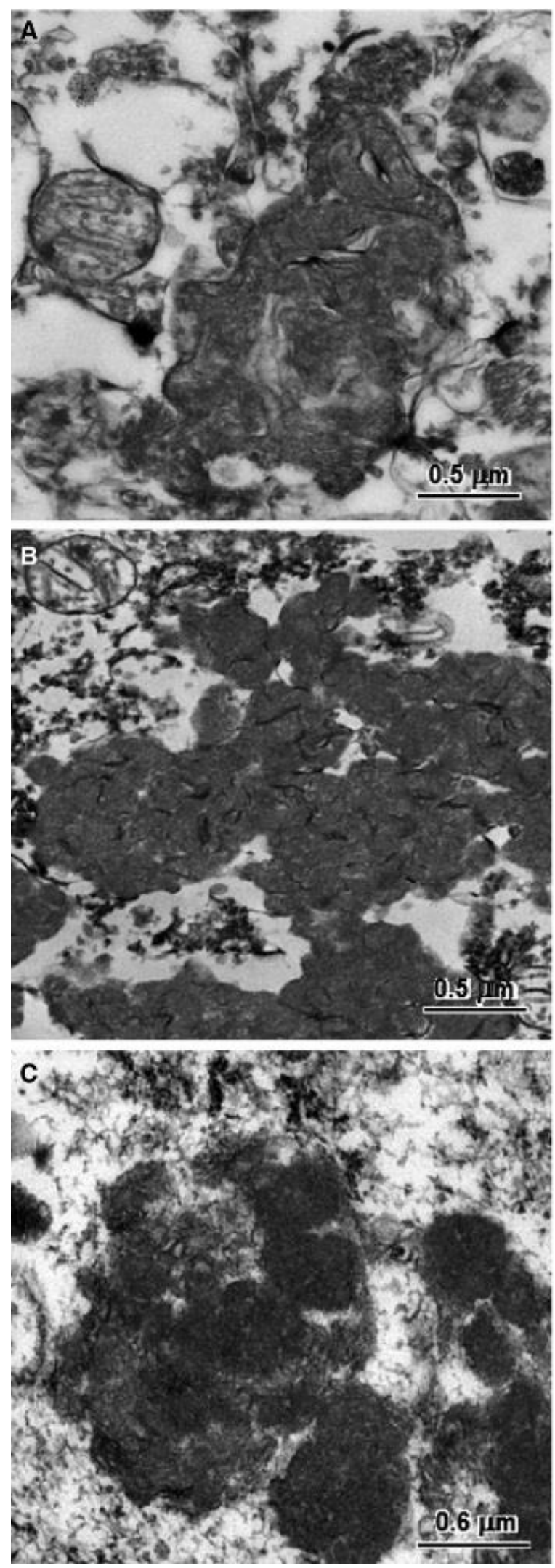

Fig. 5. Electron micrographs of the disease-specific storage bodies from a cerebellar Purkinje cells (A), a cerebral cortical neuron (B), and a retinal ganglion cell (C). 
In conjunction with the clinical signs, the light and electron microscopic results indicated the dog had NCL. To identify the molecular genetic cause of this Cane Corso's disease, we used the dog's DNA to generate a whole genome sequence. This has proven to be an efficient strategy for identifying the mutations responsible for NCL. ${ }^{51,52,82,117,143}$ Procedures for DNA isolation, library preparation, sequence generation, and sequence analysis are provided in the Supplemental Material. The affected Cane Corso's whole genome sequence had 29-fold average coverage and contained 10,965 variants predicted to alter the primary structure of the encoded proteins. Only 4 of these variants occurred within one of the 13 genes (PPT1, TPP1, CLN3, CLN5, CLN6, MFSD8, CLN8, CTSD, DNAJC5, CTSF, ATP13A2, GRN and KCTD7) ${ }^{137}$ associated with human NCL. Three of these variants were heterozygous missense mutations in ATP13A2 (Table 1) and all three had previously been reported in the canine SNPdb database. The nonreference allele frequencies for these variants in 45 control whole genome sequences from normal dogs or dogs with other diseases approached or exceeded $50 \%$ and the non-reference allele was homozygous in from 15 to 19 of these control whole genome sequences (Table 1). These common sequence variants that were heterozygous in the affected dog are therefore very unlikely to have contributed to the dog's rare recessive disease. 
Table 1. Sequence variants in neuronal ceroid lipofuscinosis-associated genes.

\begin{tabular}{|c|c|c|c|c|c|}
\hline $\begin{array}{l}\text { Chromosomal } \\
\text { Coordinate }^{\mathrm{a}}\end{array}$ & Zygosity & cDNA Change & $\begin{array}{c}\text { Amino } \\
\text { Acid } \\
\text { Change }\end{array}$ & $\begin{array}{c}\text { SNPdb } \\
\text { Reference }\end{array}$ & $\begin{array}{l}\text { Frequency } \\
\text { in Controls }\end{array}$ \\
\hline $\begin{array}{c}\text { g.81203298 } \\
\text { (CFA2) }\end{array}$ & Heterozygous & ATP13A2:c.251A>G & p.H84R & $\begin{array}{c}r s 85234162 \\
7\end{array}$ & 0.485 \\
\hline $\begin{array}{c}g: 81204154 \\
(\text { CFA2) }\end{array}$ & Heterozygous & ATP13A2:c.491T>C & p.M164T & $\begin{array}{c}r s 85107807 \\
9\end{array}$ & 0.542 \\
\hline $\begin{array}{c}g: 81215472 \\
\text { (CFA2) }\end{array}$ & Heterozygous & ATP13A2:c.3469A>G & p.M1157V & $\begin{array}{c}r s 85075182 \\
1\end{array}$ & 0.622 \\
\hline $\begin{array}{c}\mathrm{g}: 2860424 \\
(\mathrm{CFA15)}\end{array}$ & Homozygous & PPT1:c.124+1G>A & $\begin{array}{l}\text { Splice } \\
\text { variant }\end{array}$ & none & Zero \\
\hline
\end{tabular}

${ }^{a}$ CanFam3.1 genome assembly

The remaining variant, a $P P T 1: \mathrm{c} .124+1 \mathrm{G}>\mathrm{A}$ splice donor mutation, is much more likely to be the cause of the NCL in this case. The non-reference allele was homozygous in the affected dog, has not previously been reported in dbSNP, and was absent from the whole genome sequences of 45 control canines. The Integrative $\quad$ Genomics $\quad$ Viewer genome browser (https://www.broadinstitute.org/igv/) was used to visualize the variant (Fig. 6) and its validity was independently verified by Sanger sequencing (details in Supplemental Materials). The PPT1:c.124+1G>A mutation destroys the splice donor consensus motif required for exon recognition and exon-to-exon splicing by the spliceosome. ${ }^{185}$ Mutations in the "G" of the canonical "GT" or "GC" motifs found immediately 3 ' to all but the last exon in mammalian genes are the most common category of disease-causing splice site mutations. ${ }^{186}$ Mutations that weaken or destroy splice donor sites can alter exon splicing patterns in 3 ways. They can result in the skipping of the adjacent exon, the use of nearby cryptic splice sites, or the inclusion of the intron. ${ }^{187}$ The splice donor mutation in the Cane Corso with 
NCL cannot induce exon skipping because it occurs adjacent to exon 1 which also contains the initiator methionine codon. A scan of the genomic DNA sequence for potential nearby cryptic sites identified 3 "GT" motifs within 50 bp of the splice donor mutation: 2 in exon 1 with starting positions at -48 and -19 relative to the intron/exon junction and 1 in intron 1 starting at position +19 relative to the intron/exon junction. However, an internet tool (http://genes.mit.edu/burgelab/maxent/Xmaxentscan scoreseq.html) estimates the strength of splice donor signals based on maximum entropy modeling of the nucleotide sequences that surround the canonical "GT" motifs 188 indicated that none of the 3 "GT" motifs near the splice donor mutation reside in potential cryptic splice sites (Table 2). Thus, transcripts from PPT1 with the splice donor mutation are most likely to retain the 5 ' end of the $4.6 \mathrm{~kb}$ intron 1 . If these transcripts escaped nonsense mediated decay ${ }^{189}$ and were translated, they would be expected to produce a severely truncated gene product containing 42 amino acids at the $\mathrm{N}$-terminal end encoded by exon 1 followed by 114 unrelated amino acids encoded by intron 1 before the first in-frame termination codon. Nonetheless, it is highly unlikely that the mutant $P P T 1$ could produce a product that retained any biological function.

In 1995 PPT1 became the first gene reported to harbor NCL-causing mutations when 3 PPT1 mutations were identified as probable causes for NCL in 42 infants, mostly from Finland ${ }^{190}$. Since then at least 70 additional variant alleles in or around PPT1 have been reported in human NCL patients (http://www.ucl.ac.uk/ncl/CLN1mutationtable.htm). PPT1 encodes a soluble 
enzyme with palmitoyl-protein thioesterase activity. The human disease caused by PPT1 mutations is referred to as CLN1 (OMIM \#256730). Homozygous or compound heterozygous nullifying mutations in PPT1 typically produce a severe form of NCL in which neurologic signs become apparent before or during the second year of life. ${ }^{171}$ CLN1 patients seldom survive past their early teens. Hypomorphic PPT1 mutations have been associated with less aggressive CLN1 disease in older children and adults. Currently there are no medical treatments that can reverse or slow CLN1 disease progression. 


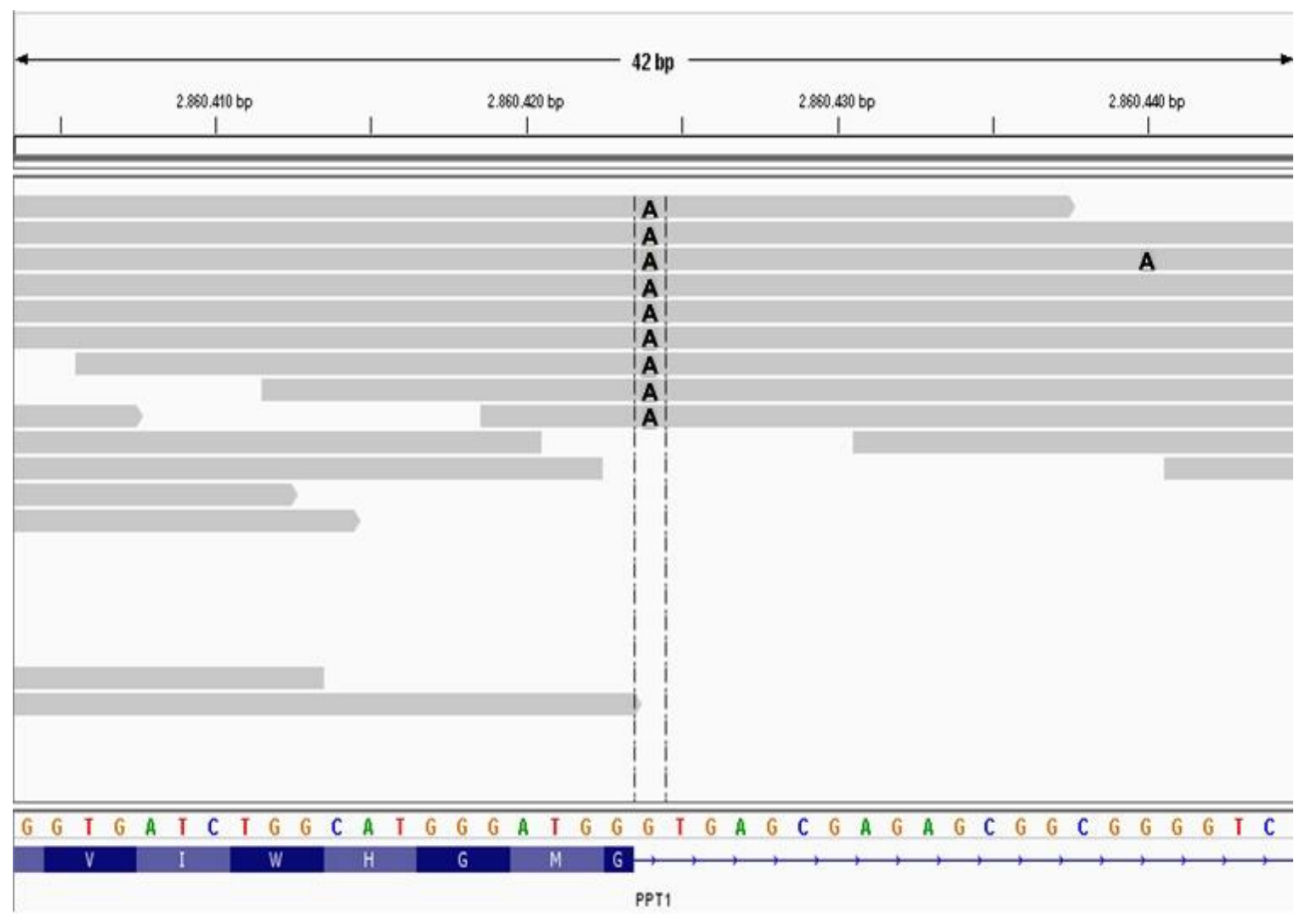

Fig. 6. A screen capture of the affected Cane Corso's whole genome sequence alignment over 42 bp of canine chromosome 15 visualized with the Integrative Genomics Viewer. Each gray bar represents sequence reads aligned to the CanFam3.1 reference sequence assembly shown with the 4-color nucleotide sequence near the bottom of the figure. Variants in the sequence reads are indicated by letters at the pertinent positions within the gray bars. In this case, the As within the gray bars indicate the variant's position where an $\mathbf{A}$ in a sequence read replaces a $\mathbf{G}$ from the reference sequence. Colors were modified from the screen capture to enhance visibility of the As. The track at the bottom shows the amino acid sequence translated from codons at the 3 ' end of PPT1 exon 1. The horizontal dashed lines enclose the homozygous As at position +1 of intron 1 that destroy the canonical GT splice donor motif.

Table 2. Palmitoyl protein thioesterase 1 exon 1 splice donor strength predictions

\begin{tabular}{|l|l|l|l|}
\hline \multicolumn{1}{|c|}{$\begin{array}{c}\text { Position of "G" in "GT" } \\
\text { motif }\end{array}$} & \multicolumn{1}{|c|}{ Motif } & \multicolumn{1}{|c|}{$\begin{array}{c}\text { Maximum Entropy } \\
\text { Score }^{\dagger}\end{array}$} & \multicolumn{1}{c|}{ Conclusion } \\
\hline c.124+1G (wild type) & tggGTGAGC & +7.23 & Potentially functional \\
\hline c.124+1G>A (mutant) & tggATGAGC & -0.95 & Not functional \\
\hline c. $77 G$ (exonic) & ggcGTCTGG & -10.70 & Not functional \\
\hline c. $106 G$ (exonic) & ctgGTGATC & +0.38 & Not functional \\
\hline c. $124+19$ (intronic) & gggGTCGGA & -3.58 & Not functional \\
\hline
\end{tabular}

†Maximum Entropy Scores range from -20 (weakest) to +20 (strongest).Motifs with scores above +3 are predicted to be potentially functional donor splice sites.

At least 4 different transgenic mouse models carrying nullifying Ppt1 mutations have been created and shown to exhibit CLN1-like disease characteristics. ${ }^{191-194}$ 
These models are the subjects of ongoing and planned evaluations of therapeutic interventions intended for human CLN1 patients. ${ }^{195-200}$ Due to differences in longevity, size, and the complexities of neuroanatomy and behavior, canine NCL models have some advantages over rodent models for optimizing therapeutic interventions or establishing their efficacy. Indeed, a TPP1-deficient canine model for the CLN2 form of NCL has been used to develop enzyme replacement therapy ${ }^{182}$ and paved the way for ongoing human trials (https://clinicaltrials.gov/ct2/show/NCT02678689).

A previous report described a different homozygous canine PPT1 mutation (PPT1:c.736_737insC) in a Dachshund with NCL. ${ }^{140}$ The Dachshund PPT1 frameshift mutation was predicted to encode a truncated gene product. Brain tissue from the affected Dachshund had only trace thioesterase activity, attributed to other thioesterases such as that encoded by PPT2. ${ }^{140}$ In both the Dachshund CLN1 case and the currently described Cane Corso CLN1 case, the initial neurodegenerative changes became apparent when the dogs were approximately 8 months old. Both dogs were euthanized because of their increasingly severe neurologic signs. The Dachshund was euthanized at 14 months of age; whereas, the Cane Corso was euthanized when only 10 months old. The earlier euthanasia of the much larger Cane Corso may have been influenced by difficulties caring for a large dog with deteriorating neurologic function. The NCL in both the Cane Corso and the PPT1-deficient Dachshund had an early onset and rapid progression similar to that of TPP1-deficient Dachshunds, but was earlier and more rapid than has been reported in dogs with other types of NCL (Table 3). A similar association 
between the disease-causing mutations and disease phenotype was recently confirmed by comparing the various types of human NCL. ${ }^{201}$

Tissues from the CLN1 affected Dachshund were examined by electron microscopy. ${ }^{140}$ In that case, distinct membranes were observed to at least partially surround the storage material aggregates. As with the Cane Corso tissue, the initial fixation of the Dachshund tissue was in formalin; however, the partial preservation of the surrounding membranes in the case of the Dachshund was probably due to a shorter time interval between the initial fixation and the transfer to the glutaraldehyde fixative. Better ultrastructural preservation of the storage bodies in future cases will require initial fixation in glutaraldehyde at short postmortem times. In conclusion, our findings indicate a novel splice donor mutation causing CLN1 in a dog breed previously unreported to possess the disease. A canine model for CLN1 disease could provide opportunities to further the development of therapeutic options, benefiting affected humans and canines. To find a potential founder for a colony of PPT1-deficient research dogs, all 5 Cane Corso DNA samples from our DNA repository and 26 Cane Corso DNA samples provided by MARS Veterinary ${ }^{\mathrm{TM}}$ were screened for the PPT1c.124+1A allele with a modified allelic discrimination assay that is described in the Supplemental Material. All 31 Cane Corso DNA samples tested homozygous for the reference PPT1c.124+1G allele. In a continuing effort to identify dogs that could help develop effective treatments for infants with CLN1 disease and to assist breeders in avoiding the production of affected puppies, we are offering free DNA testing for Cane Corsos or Dachshunds showing visual deterioration and neurodegenerative changes 
starting around or before 8 months of age. Arrangements for free testing can be made with Martin Katz (katzm@health.missouri.edu). 
Table 3. Ages at disease onset and euthanasia for dogs with genetically defined neuronal ceroid lipofuscinosis (NCL)

\begin{tabular}{|c|c|c|c|c|c|c|}
\hline Disease & Gene & Mutation & Breed & $\begin{array}{c}\text { Age at } \\
\text { Onset } \\
\text { (months) }^{\dagger}\end{array}$ & $\begin{array}{c}\text { Age at } \\
\text { Euthanasia } \\
\text { (months) }\end{array}$ & Reference \\
\hline CLN1 & PPT1 & c. $124+1 G>A$ & $\begin{array}{l}\text { Cane } \\
\text { Corso }\end{array}$ & 8 & 10 & Current \\
\hline CLN1 & PPT1 & c.736_737insC & Dachshund & 8 & 14 & 140 \\
\hline CLN2 & CLN2 & c.325delC & Dachshund & $7-9$ & 12 & 141 \\
\hline CLN5 & CLN5 & $c .619 C>T$ & $\begin{array}{l}\text { Border } \\
\text { Collie, } \\
\text { Australian } \\
\text { Cattle Dog }\end{array}$ & $12-23$ & $15-29$ & $82,142,163$ \\
\hline CLN5 & CLN5 & c.934_935delAG & $\begin{array}{l}\text { Golden } \\
\text { Retriever }\end{array}$ & $13-17$ & $30-34$ & 143 \\
\hline CLN6 & CLN6 & c.829T>C & $\begin{array}{l}\text { Australian } \\
\text { Shepherd }\end{array}$ & 18 & 24 & 144 \\
\hline CLN7 & MFSD8 & c.843delT & $\begin{array}{l}\text { Chinese } \\
\text { Crested, } \\
\text { Chihuahua }\end{array}$ & 12 & $16-24$ & 51,117 \\
\hline CLN8 & CLN8 & c. $491 \mathrm{~T}>\mathrm{C}$ & $\begin{array}{l}\text { English } \\
\text { Setter }\end{array}$ & $12-14$ & $24-27$ & 145 \\
\hline CLN8 & CLN8 & c. $585 \mathrm{G}>\mathrm{A}$ & $\begin{array}{l}\text { Australian } \\
\text { Shepherd }\end{array}$ & 11 & 21 & 52 \\
\hline CLN10 & CTSD & $c .585 G>A$ & $\begin{array}{l}\text { American } \\
\text { Bulldog }\end{array}$ & $11-36$ & 42 to $66^{\ddagger}$ & 146,202 \\
\hline CLN12 & ATP13A2 & c. $1623 G>A$ & $\begin{array}{l}\text { Tibetan } \\
\text { Terrier }\end{array}$ & $60-96$ & Up to 120 & 147,148 \\
\hline
\end{tabular}




\section{Supplemental material}

\section{Generation and analysis of the whole genome sequence:}

The DNA was extracted from blood from the affected Cane Corso as previously described ${ }^{145}$ and submitted to the University of Missouri DNA Core Facility for library preparation and sequencing. A PCR-free DNA sample preparation kit $^{\mathrm{a}}$ was used to prepare two libraries with approximate lengths of $350 \mathrm{bp}$ and $550 \mathrm{bp}$. Separate 2X100 bp paired-end sequences were generated in individual flow-cell lanes with a massively parallel DNA sequencer. ${ }^{b}$ Trimmomatic $^{203}$ software was used for removal of the adaptor sequences. This sequence data was deposited into the Sequence Read Archives (SRS1124447). The reads were aligned to the canine genome reference sequence (CanFam 3.1) using the default parameters of the bwa mem algorithm from the BWA 0.7 .12 software. ${ }^{157}$ The Samtools 1.2 software ${ }^{158}$ was used to convert file formats, merge libraries, sort and index the alignment files by location. The default parameters of the Platypus 0.8 .1 variant caller software ${ }^{159}$ was used for variant calling. The variant annotation and filtering was done using a commercial software ${ }^{\mathrm{C}}$ Another 45 whole genome sequences generated and processed as described above were used to estimate allele frequencies of candidate sequence variants. The breeds of origin and the SRA accession numbers for these 45 whole genome sequences are provided in the Table at the end of this section. Specific chromosomal regions of the affected Cane Corso alignment were inspected using the Integrative Genomics Viewer software to evaluate the quality of the alignment surrounding candidate sequence variants. ${ }^{204}$ The existence of a potentially causal homozygous variant, 
PPT1:c.124+1G>A, was confirmed by direct Sanger sequencing of an amplicon produced with flanking PCR primers 5'-GCTGCCGCTGGTGATCT-3' and 5'AGACGCGAACCCAGGTC-3'.

\section{Genotyping individual canine DNA samples for the PPT1:c.124+1G>A transition:}

Initial attempts to genotype individual canine DNA samples for the PPT1:c.124+1G>A transition with a TaqMan allelic discrimination assay ${ }^{81}$ were unsuccessful, probably due to the repetitive and GC-rich character of sequences adjacent to the candidate transition. We therefore made use of a previously described nested primer modification. ${ }^{205}$ A pre-amplification step was done with a commercial kit designed for PCR amplification of GC-rich templates ${ }^{d}$ The PCR primers were 5'-GGTCACGTGATCGGGCCAAGA-3' and 5'GCCAGTCCCCGCTCCCGTCT-3'and the pre-amplification was accomplished with 18 cycles at an annealing temperature of $650 \mathrm{C}$. For the TaqMan allelic discrimination assay, $2 \mu \mathrm{l}$ (out of the initial $30 \mu \mathrm{l}$ pre-amplication reaction mixture) was amplified using the forward primer 5'-GCTGCCGCTGGTGATCT-3', reverse primer 5'-AGACGCGAACCCAGGTC-3'. The competing TaqMan probes were 5'VICATGGGATGGGTGAGCGA-MGB-3' (reference allele) and 5'-FAMATGGGATGGATGAGCGA-MGB-3' (variant allele). 


\section{Supplemental Table 1: Breeds and SRA numbers for the control whole genome sequences}

\begin{tabular}{|c|c|}
\hline Breed & SRA Number \\
\hline Standard Poodle & SRS932171 \\
\hline Airedale & SRS932170 \\
\hline Basenji & SRS1124466 \\
\hline Soft Coated Wheaten Terrier & SRS834052 \\
\hline Kerry Blue Terrier $\mathrm{x}$ beagle cross & SRS1124465 \\
\hline Labrador Retriever & SRS1124463 \\
\hline Chinook & SRS932169 \\
\hline Pembroke Welsh Corgi & SRS732549 \\
\hline Pembroke Welsh Corgi & SRS732550 \\
\hline Pointer & SRS932168 \\
\hline Jack Russell Terrier & SRS932167 \\
\hline Italian Greyhound & SRS932166 \\
\hline Saint Bernard & SRS932165 \\
\hline Portuguese Podengo & SRS932163 \\
\hline Border Collie & SRS932162 \\
\hline English Springer Spaniel & SRS932160 \\
\hline German Shepherd & SRS932159 \\
\hline Basenji & SRS932158 \\
\hline Labrador Retriever & SRS932152 \\
\hline Jack Russell Terrier & SRS932151 \\
\hline Tibetan Terrier & SRS932148 \\
\hline Jack Russell Terrier & SRS932147 \\
\hline Berger Picard & SRS932146 \\
\hline Tibetan Terrier & SRS932145 \\
\hline Border Terrier & SRS932144 \\
\hline Standard Poodle & SRS932143 \\
\hline Shetland Sheepdog & SRS932138 \\
\hline Berger Picard & SRS1124462 \\
\hline Airedale Terrier & SRS1124461 \\
\hline Soft Coated Wheaten Terrier & SRS834054 \\
\hline Jack Russell Terrier & SRS1124459 \\
\hline English Cocker Spaniel & SRS834057 \\
\hline Black \& Tan Coonhound & SRS1124458 \\
\hline Golden Retriever & SRS1124457 \\
\hline Border Terrier & SRS1124456 \\
\hline Border Terrier & SRS1124455 \\
\hline English Springer Spaniel & SRS1124454 \\
\hline English Setter & SRS1124453 \\
\hline Gordon Setter & SRS1124452 \\
\hline Berger Picard & SRS1124451 \\
\hline Shiba Inu & SRS1124450 \\
\hline Labrador Retriever & SRS1124449 \\
\hline Golden Retriever & SRS834021 \\
\hline Australian Cattle Dog & SRS834022 \\
\hline Lowchen & SRS1124448 \\
\hline
\end{tabular}




\section{Footnotes}

a TruSeq DNA PCR-Free Sample Preparation Kit, Illumina, San Diego, CA

b HiSeq 2500 System, Illumina, San Diego, CA

c SNP \& Variation Suite v8.x, Golden Helix, Inc., Bozeman, MT

d AccuPrimeTM GC-rich kit, Invitrogen, Carlsbad, CA 92008, USA 


\section{Chapter 5}

\section{GM2 gangliosidosis in Shiba Inu dogs with an inframe deletion in $H E X B$}

\section{Abstract}

Consistent with a tentative diagnosis of neuronal ceroid lipofuscinosis (NCL), autofluorescent cytoplasmic storage bodies were found in neurons from the brains of 2 related Shiba Inu dogs with a young-adult onset, progressive neurodegenerative disease. Unexpectedly, no potentially causal NCL-related variants were identified in a whole genome sequence generated with DNA from 1 of the affected dogs. Instead, the whole genome sequence contained a homozygous 3 base pair (bp) deletion in a coding region of HEXB. The other affected dog also was homozygous for this 3 bp deletion. Mutations in the human HEXB ortholog cause Sandhoff disease, a type of GM2 gangliosidosis. Thin layer chromatography confirmed that GM2 ganglioside had accumulated in an affected Shiba Inu brain. Enzymatic analysis confirmed that the GM2 gangliosidosis resulted from a deficiency in the $H E X B$ encoded protein and not from a deficiency in products from HEXA or GM2A, which are known alternative causes of GM2 gangliosidosis. We conclude that the homozygous $3 \mathrm{bp}$ deletion in HEXB is the likely cause of the Shiba Inu neurodegenerative disease and that whole genome sequencing can lead to the early identification of potentially disease-causing DNA variants thereby refocusing subsequent diagnostic analyses towards confirming or refuting candidate variant causality. 


\section{Introduction}

The clinical records of 2 young-adult Shiba Inu dogs that exhibited similar signs of progressive, fatal, neurodegeneration came to our attention from independent sources. Pedigree inspection indicated that both dogs shared at least 1 common maternal and paternal ancestor, as illustrated in Fig. 1. Thus, it appeared that the same recessively inherited neurodegenerative disease affected both dogs. We used whole genome sequencing to efficiently identify a genetic variant that was most likely responsible for this disease and to reach a highly probable, but unexpected, diagnosis.

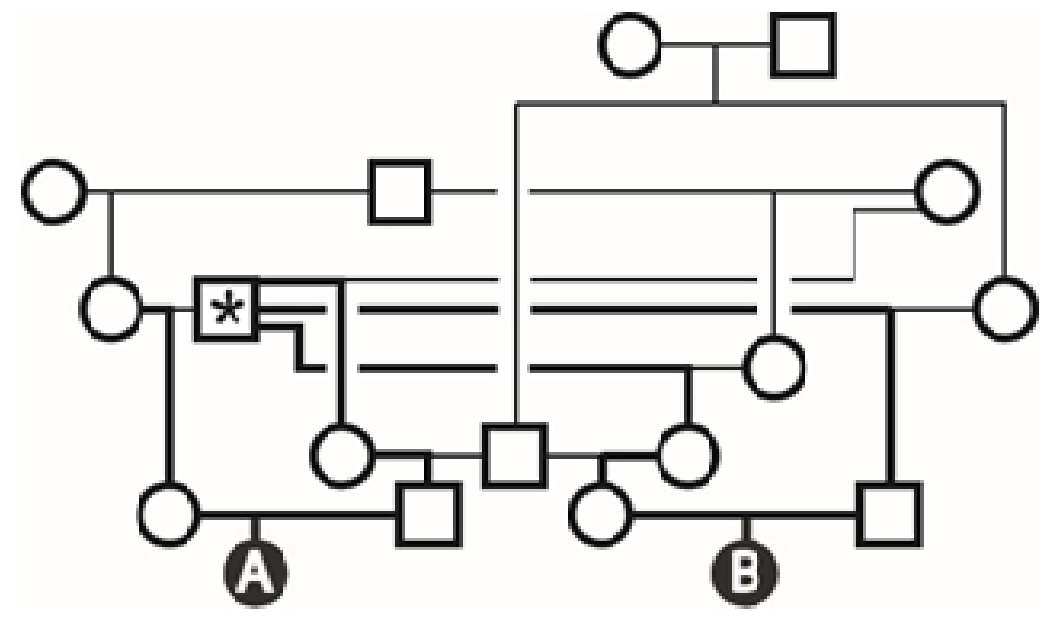

Fig.1 Pedigree shows consanguineous familial relationships for the 2 affected Shiba Inu, Dog A and Dog B. The dog marked with an asterisk contributed genetic material to both parents of both affected dogs. Bold lines show the shortest possible pathway for the inheritance of the causal allele from an ancestor to the homozygous affected dogs. Nonetheless, we do not have DNA from the ancestors to verify this pathway and additional consanguineous relationships among earlier ancestors are likely. Thus, Dog A and Dog B may have obtained their homozygous mutant allele by alternative inheritance pathways. 


\section{Methods}

The DNA from 1 affected Shiba Inu (Dog A) was submitted to a commercial sequencing center $^{\mathrm{a}}$ for PCR-free library preparation and sequencing with a commercial sequencer ${ }^{b}$ that produced paired-end 150 bp reads. Details about the pipeline used to process and analyze this data set are available in the supplementary material. The PCR primers 5'-AAATAGTATAATCATGTGCAA-3' and 5'-CCCAAATACATTTACTGCCTA-3' were used to produce amplicons containing a candidate variant identified in the whole genome sequence. These amplicons were analyzed by Sanger sequencing for validation and by PCRrestriction fragment length polymorphism (PCR-RFLP) with Mnll for genotyping archived Shiba Inu DNA. Previously described procedures were used for fluorescence and electron microscopy ${ }^{52}$ and for thin layer chromatography and enzymology. ${ }^{117}$

\section{Clinical Findings}

Dog A, a spayed female Shiba Inu, exhibited progressive neurologic signs starting with generalized tremors and altered tail carriage (tail down) when approximately 12 months old. As the disease progressed, the severity of the tremors increased and the dog exhibited marked cerebellar ataxia, spastic tetraparesis, anxiety, decreased appetite, decreased responsiveness to verbal commands, trance-like behavior, loss of ability to climb up and down stairs, and impaired vision. Magnetic reasonance imaging (MRI) of the head showed a diffuse loss of gray-white matter distinction on T2-weighted imaging with white matter appearing isointense to 
marginally hypointense relative to grey matter. Because of the progression of neurological signs, the dog was euthanized at approximately 17 months of age.

Dog B also was a spayed female Shiba Inu. Similar to Dog A, the first clinical signs in Dog B appeared at about 12 months of age and included situational anxiety, inability to climb up or down stairs, a wide-based stance, severe generalized tremors, and the same alteration in tail carriage shown by Dog A. During a neurologic examination at 15 months of age, Dog B had normal proprioception but showed intention tremors and severe cerebellar ataxia with frequent falls. The menace response was decreased to absent. A brain MRI, including T2- and T1weighted sequences before and after administration of a contrast agent, showed decreased gray-white matter distinction in both the cerebrum and cerebellum similar to Dog A. In the cerebellum, arbor vitae could not be identified on the sagittal T2-weighted sequence. The ventricular system was symmetrical and normal in size. The neurological signs progressed to include anxiety, compulsive panting, trance-like behavior, intolerance to loud noise, and loss of training including house training. Dog B developed severe muscle stiffness and weakness that spread from the hind limbs resulting in ataxia and frequent falls. In addition, Dog B showed severe visual impairment in both bright and dim light. A sleep disturbance with leg movements mimicking running started at about 18 months of age. Near the end of its life, Dog B was unable to walk or eat without assistance. Dog B died while asleep when approximately 20 months old. Commercial laboratories $^{c, d}$ determined that neither affected dog had a GLB1 frameshift 
mutation, chr23:3,796,317delC (CanFam 3.1), known to cause GM1 gangliosidosis in the Shiba Inu breed (OMIA 000402-9615). ${ }^{206}$

Because the clinical signs suggested neuronal ceroid lipofuscinosis (NCL) as a possible cause, blood and other tissue samples were shipped to the University of Missouri for further analysis. Fluorescence microscopy of unstained tissue sections from both affected dogs identified massive accumulations of yellowemitting autofluorescent storage bodies in neurons throughout the cerebellum (Fig. 2A), the cerebral cortex (Fig. 2B) and the retina (Fig. 2C).
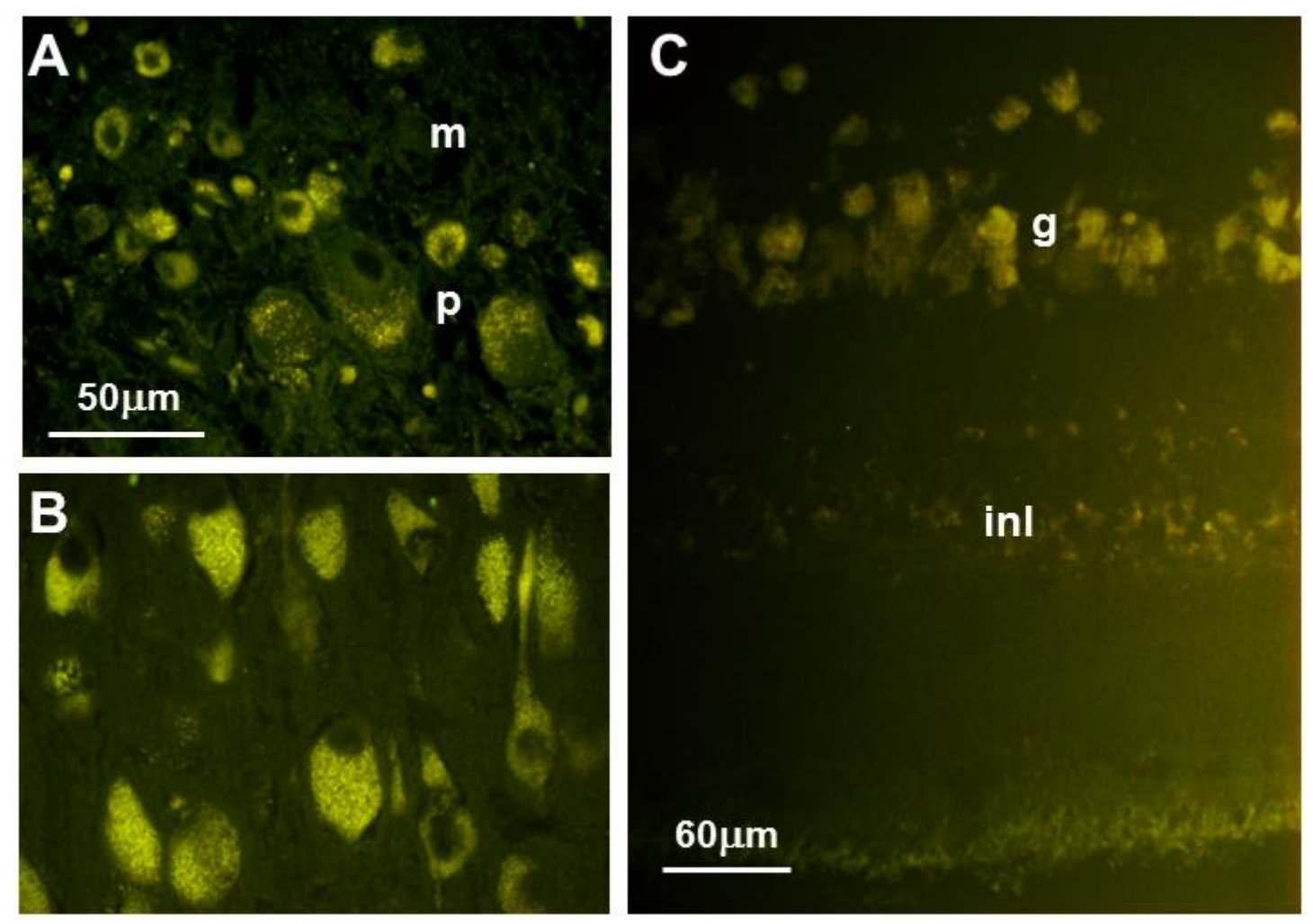

Fig. 2 Fluorescence micrographs of unstained sections of cerebellum (A), cerebral cortex (B), and retina (C) from an affected Shiba Inu. In the cerebellum, autofluorescent intracellular inclusions were present in both the Purkinje cell $(p)$ and molecular $(m)$ layers. Neurons throughout the cerebral cortex gray matter had abundant autofluorescent inclusions. In the retina, the most pronounced accumulation of autofluorescent material was seen in the ganglion cell layer (g), but some accumulation was also present in the inner nuclear layer (inl). Bars in (A) and (B) indicate the magnification for the micrographs. 


\section{Molecular-genetic diagnosis}

The clinical signs and fluorescent microscopic results were consistent with a diagnosis of NCL. Because whole genome sequencing previously had proven to be an effective strategy for identifying genetic variants responsible for NCL in dogs, ${ }^{51,52,54,82,117,143}$ a whole genome sequence was generated with DNA from Dog A. The resulting sequence data had 31-fold average coverage of the CanFam3.1 reference assembly and is available in the National Center for Biotechnology Information Sequence Read Archives (accession SRS1692083). As we have done previously, ${ }^{52,82,117,143}$ the aligned reads from the whole genome sequence were scanned for rare variants in or near the coding exons of canine orthologs of the 13 genes currently associated with human NCL: PPT1, TPP1, CLN3, CLN5, CLN6, MFSD8, CLN8, CTSD, DNAJC5, CTSF, ATP13A2, GRN and KCTD7.137 Unexpectedly, we did not find plausible candidate variants. In addition, the alignment was checked for a GLB1 frameshift mutation, chr23:3,796,317delC (CanFam 3.1), known to cause a GM1 gangliosidosis in the Shiba Inu, ${ }^{206}$ but only the reference allele was found confirming antemortem DNA test results. The search for a causal genetic variant was expanded by filtering the millions of sequence variants found in the Shiba Inu whole genome sequence to retain only those that were: (1) predicted to affect the primary structure of a gene product, (2) homozygous in the affected Shiba Inu, and (3) absent from the 105 whole genome sequences of normal dogs or dogs with other diseases. Only 80 variants in 74 different genes met these criteria (see Supplementary Table 1). Brief searches for the known biological functions of these 74 genes in OMIM 


\section{(https://www.ncbi.nlm.nih.gov/omim)}

and

in

PubMed

(https://www.ncbi.nlm.nih.gov/pubmed) identified a 3 bp deletion in HEXB as the sequence variant considered most likely to be responsible for the Shiba Inu disorder because it was the only variant in a gene that has previously been associated with lysosomal storage disease. The chromosomal location of this deletion is chr2:57,243,656_57,243,658delCCT (CanFam 3.1).

Sanger sequencing confirmed that both affected Shiba Inu were homozygous for the $3 \mathrm{bp} H E X B$ deletion. The absence of this deletion from our 105 control whole genome sequences suggested that it is uncommon in dogs in general. To estimate the frequency of the $3 \mathrm{bp} H E X B$ deletion among the Shiba Inu, all 40 Shiba Inu represented in the University of Missouri DNA repository were genotyped by PCRRFLP for this deletion. Thirty-seven of the tested Shiba Inu were homozygous for the reference allele; the other 3 were heterozygotes. These heterozygotes were born in 2002, 2004, and 2007. There are no known familial relationships among them or between them and the 2 affected Shiba Inu described here. This observation suggests that the $3 \mathrm{bp} H E X B$ deletion may be rare but widely distributed in the Shiba Inu breed.

\section{Biochemical and electron microscopic confirmation}

The HEXB deletion predicts the loss of a leucine residue (p.Leu317del; XP_005617969.1) in the encoded $\beta$-subunit of the $\beta$-hexosaminidase enzyme complex. A leucine residue occurs at a homologous position in $\beta$-subunits from 53

other mammalian species (see Supplementary Figure 1). A functional $\beta$-subunit 
provides essential glycosidic activity to the $\beta$-hexosaminidase enzyme complex that removes the terminal $\mathrm{N}$-acetyl-galactosamine from GM2 ganglioside, an essential step in the lysosomal degradation of gangliosides. Genetic variants in human $H E X B$ can result in a $\beta$-hexosaminidase deficiency called Sandhoff disease and cause the accumulation of GM2 ganglioside..$^{207}$

Thin layer chromatography was used to determine if GM2 ganglioside had accumulated in brain gray matter from 1 of the affected Shiba Inu (Dog B). The resulting thin layer chromatogram is shown in Fig. 3. An extract from the brain of a human patient with GM2 gangliosidosis known to contain GM2 ganglioside as the major component was fractionated in Lane 1. The brain extract in lane 3 was from a human GM1 gangliosidosis patient. GM1 ganglioside was the major component in this extract. Lane 4 contained a normal bovine brain extract and served to show that GM1 and GM2 gangliosides do not accumulate in healthy brains. The extract in lane 2 was from one of the affected Shiba Inu (Dog B). The migration of the major component in this lane matched that in lane 1, indicating that it is also GM2 ganglioside and that the dog had a GM2 gangliosidosis. 


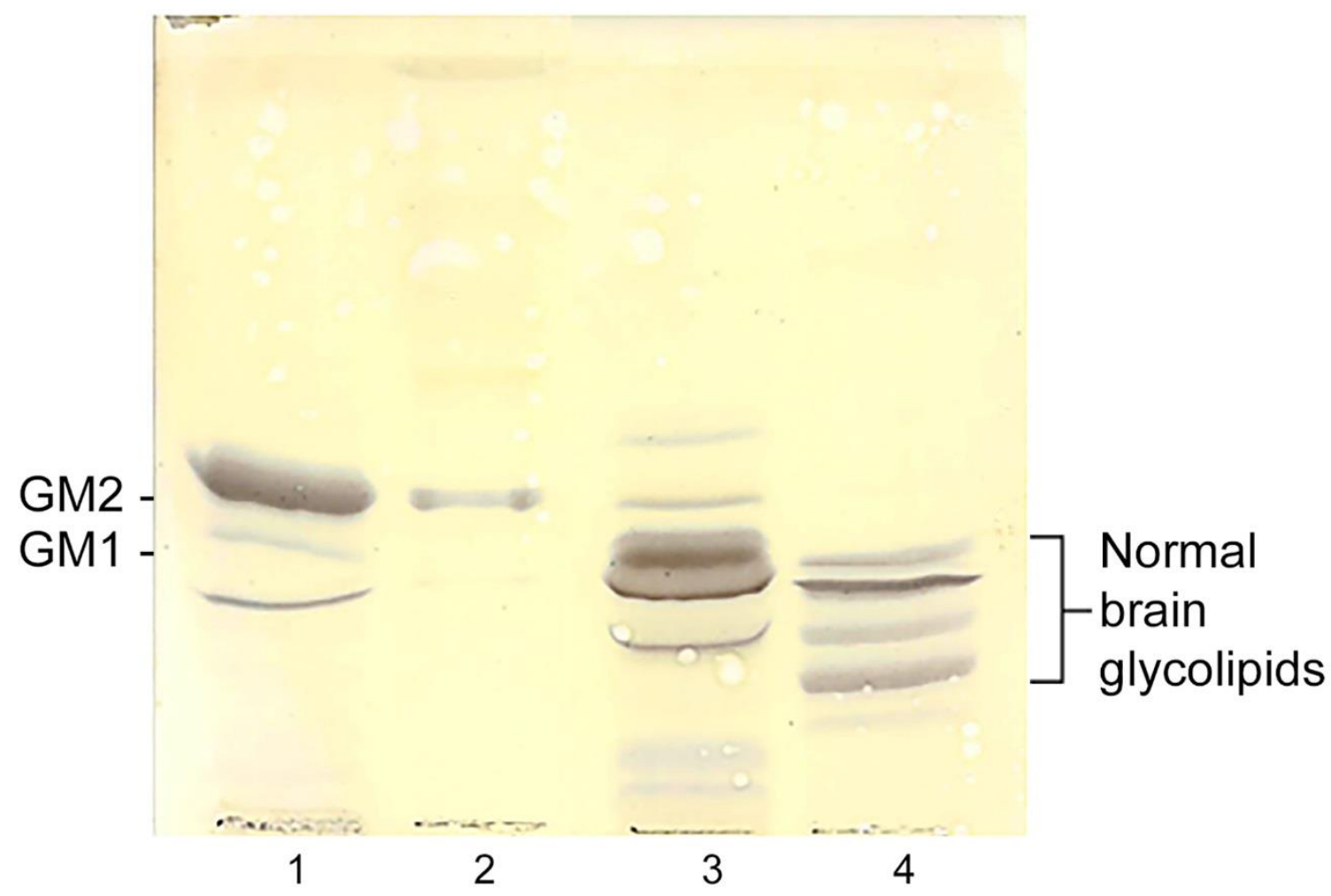

Fig.3 Thin layer chromatographic fractionation of brain gangliosides extracted from the gray matter of a human patient who died from Tay-Sachs disease (lane 1), the gray matter of Dog B, the affected Shiba Inu dog (lane 2), the gray matter of a human patient who died with GM1 gangliosidosis (lane 3) and gangliosides extracted from a normal bovine brain obtained from a commercial source (lane 4). The most abundant component in lane 1 is GM2 ganglioside (GM2). In lane 2, the most abundant component co-migrated with the GM2 ganglioside in lane 1 and not with GM1 ganglioside (GM1), the most abundant component in lane 3. Only trace amounts of GM2 ganglioside were detected in Lane 3 and none was detected in extracts from the normal brain in lane 4.

Besides HEXB, GM2 gangliosidosis can be caused by genetic variants in 2 other genes: HEXA that encodes the $\alpha$-subunit of the $\beta$-hexosaminidase enzyme complex and GM2A that encodes GM2 activator protein. Although the activator protein does not possess intrinsic enzymatic activity, its presence is required for efficient enzymatic degradation of GM2 ganglioside by $\beta$-hexosaminidase. ${ }^{208}$ The $\alpha$ - and $\beta$-subunits of $\beta$-hexosaminidase are enzymatically active as homo- or heterodimers. The 3 genetic causes of GM2 gangliosidosis can be enzymologically 
distinguished with synthetic substrates that identify the source of the residual hexosaminidase activities in patients' tissues. ${ }^{207}$ Patients with GM2 gangliosidosis caused by a GM2A deficiency have residual activity from both the $\alpha$ - and $\beta$ subunits. Thus, they are classified as $A B$ variants. Human patients with $H E X A$ deficiency (known as Tay-Sachs disease) are considered B variants because they have only $\beta$-subunit activity. Homodimers of the $\alpha$-subunit are unstable and heat labile. Patients with Sandhoff disease (HEXB deficiency) who lack the $\beta$-subunit necessary to form stable heterodimers, can only form unstable $\alpha$-subunit homodimers and thus have subnormal activities from both the $\alpha$ - and $\beta$-subunits. ${ }^{207}$ These patients are considered to be 0 (zero) variants. Table 1 contains a summary of the genetic causes, the subunit activities and the associated nomenclature for the 3 causes of GM2 gangliosidosis.

Table 1: Types of GM2 Gangliosidosis

\begin{tabular}{|l|l|l|l|l|}
\hline Disease & Gene $^{\dagger}$ & Encoded Protein & Residual Activity $^{\text {Variant }}{ }^{\ddagger}$ \\
\hline Tay-Sachs Disease & HEXA & $\begin{array}{l}\text { a-Subunit of } \beta \text { - } \\
\text { Hexosaminidase }\end{array}$ & $\beta$-Subunit & B Variant \\
\hline Sandhoff disease & HEXB & $\begin{array}{l}\beta \text {-Subunit of } \beta \text { - } \\
\text { Hexosaminidase }\end{array}$ & None & 0 Variant \\
\hline GM2 Activator Deficiency & GM2A & GM2 Activator & $\alpha$ - and $\beta$-Subunits & AB variant \\
\hline
\end{tabular}

†Harboring causal variants; ${ }^{\ddagger}$ Based on residual activity; ${ }^{\ddagger}$ None because $\alpha$-Subunit dimers are unstable

We used previously described procedures ${ }^{209}$ and the synthetic substrate, 4methylumbelliferyl $\mathrm{N}$-acetyl- $\beta$ - $\mathrm{d}$-glucosaminide, to estimate total $\beta$ hexosaminidase activity in cerebral cortical gray and white matter extracts. We also measured $\beta$-galactosidase activity in these extracts as a control. As indicated in Table 2, the total $\beta$-hexosaminidase activities in the extracts from Dog B were 
markedly decreased compared to 2 control dogs. Furthermore, the $\beta$ hexosaminidase activities in the extracts from Dog B were completely heat labile as would be expected for $\alpha$-subunit homodimers. These results are similar to those observed with 0 variant or Sandhoff disease patients and provide further evidence that the HEXB deletion identified by whole genome sequencing is responsible for the progressive neurodegeneration in the 2 affected Shiba Inu dogs.

Table 2: Enzyme Activities

\begin{tabular}{|c|c|c|c|c|c|c|}
\hline \multirow{2}{*}{ Enzyme } & \multicolumn{5}{|c|}{ Enzymatic Activities (nmol/h/mg protein) } \\
\cline { 2 - 7 } & \multicolumn{2}{|c|}{ Affected Dog B } & \multicolumn{2}{c|}{ Control Dog 1 } & \multicolumn{2}{c|}{ Control Dog 2 } \\
\cline { 2 - 7 } & $\begin{array}{c}\text { Gray } \\
\text { Matter }\end{array}$ & $\begin{array}{c}\text { White } \\
\text { Matter }\end{array}$ & $\begin{array}{c}\text { Gray } \\
\text { Matter }\end{array}$ & $\begin{array}{c}\text { White } \\
\text { Matter }\end{array}$ & $\begin{array}{c}\text { Gray } \\
\text { Matter }\end{array}$ & $\begin{array}{c}\text { White } \\
\text { Matter }\end{array}$ \\
\hline$\beta$-galactosidase & 269 & 174 & 67.1 & 25.5 & 58.9 & 31.8 \\
\hline $\begin{array}{c}\beta \text {-hexosaminidase } \\
(\% \text { Heat labile) }\end{array}$ & $\begin{array}{c}15.1(10 \\
0 \%)\end{array}$ & $\begin{array}{c}11.3 \\
(100 \%)\end{array}$ & $\begin{array}{c}663 \\
(77 \%)\end{array}$ & $\begin{array}{c}363 \\
(72 \%)\end{array}$ & $\begin{array}{c}669 \\
(79 \%)\end{array}$ & $\begin{array}{c}427(79 \% \\
)\end{array}$ \\
\hline
\end{tabular}

tRun as control

Electron microscopic examination of the storage bodies in the brain and retina showed that many neurons contained membrane-like structures arranged in loosely associated concentric whorls (Fig. 4A, C and D). Inclusions with this ultrastructural feature traditionally are called membranous cytoplasmic granules. ${ }^{207,208,210,211}$ Other less common neuronal inclusions appeared to be vacuoles with only a small amount of dispersed electron-dense material (Fig. 4B). Abnormal inclusions with the membranous cytoplasmic granules ultrastructure are common in human patients with GM2 gangliosidosis. ${ }^{210,212,213}$ In addition, membranous storage granules have been found in neurons from patients with GM1 gangliosidosis or mucopolysaccharidosis. ${ }^{213,214}$ However, they have not been reported in NCL-affected brains, which instead typically contain inclusions with 
granular, curvilinear, rectilinear, or fingerprint profiles, either singularly, or in combination..$^{215}$
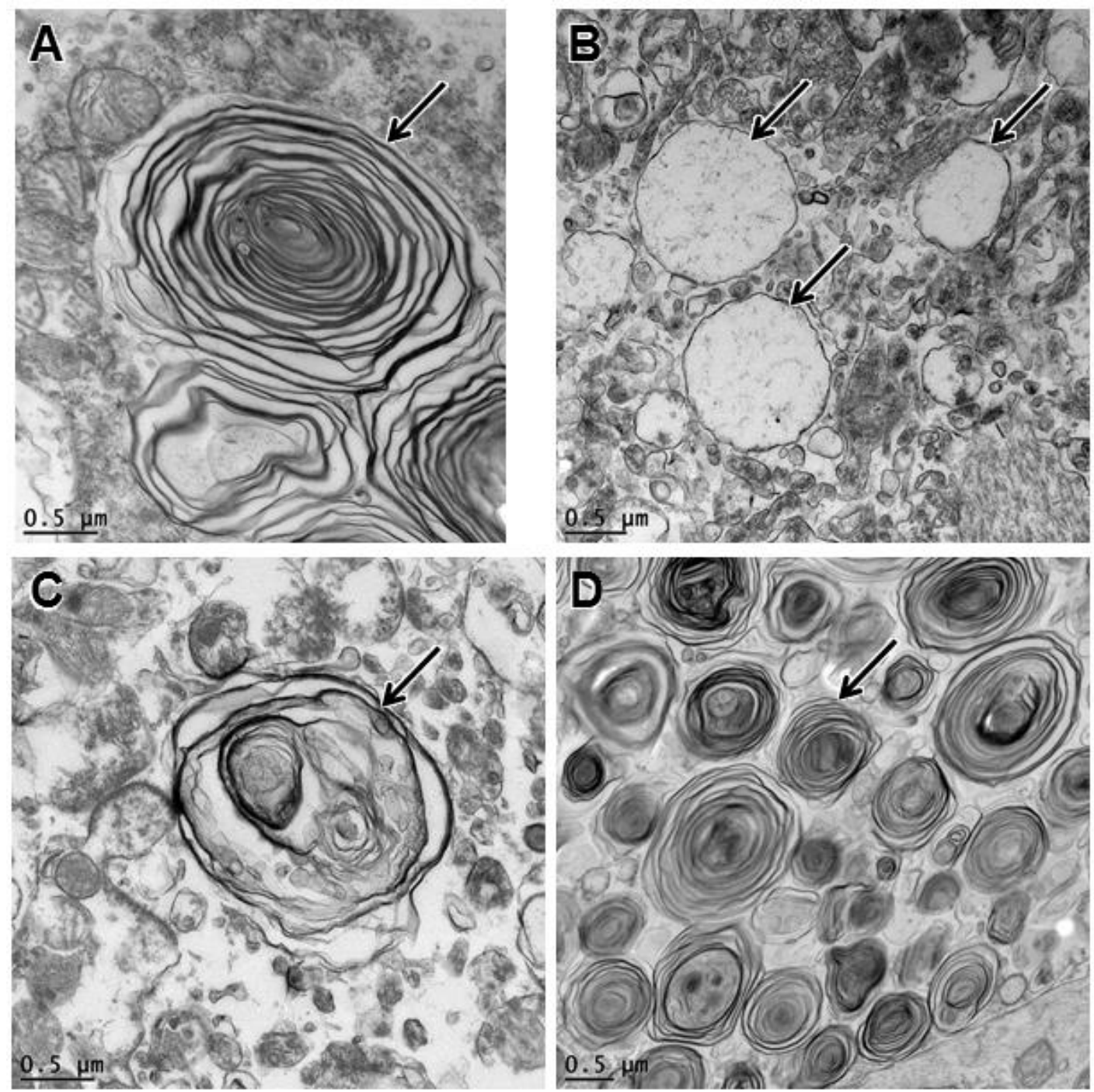

Fig.4 Electron micrographs of storage material in the cerebral cortex (A and B), cerebellar molecular layer cell (C), and retinal ganglion cells (D) of Dog B. Representative storage bodies are indicated by arrows. In cerebral cortical neurons, the storage material consisted of both concentric whorls of membrane-like structures (A) and membrane-bound vacuole-like inclusion bodies (B). In the cerebellum $(\mathbf{C})$, the storage bodies contained more irregularly arranged membrane-like material. The ultrastructural appearance of the cerebellar storage bodies was similar in both the molecular layer and Purkinje cells. In the retinal ganglion cells (D), the storage material consisted primarily of concentric whorls of membrane-like structures. 


\section{Autofluorescent storage bodies in NCL and other diseases}

Before we evaluated the whole genome sequence, we suspected that the affected Shiba Inu dogs had NCL because of their clinical signs of progressive neurodegeneration and the presence of autofluorescent storage bodies. Progressive neurodegeneration and accumulation of autofluorescent lysosomal storage bodies within neurons are widely recognized disease characteristics required for inclusion among the diverse diseases classified as NCLs. ${ }^{137}$ Nonetheless, in a few cases, disorders with these characteristics have been classified as other types of lysosomal storage diseases because of the biochemical composition of the associated storage granules or the nature of the associated enzyme deficiency or both. Among these are GM1 gangliosidosis and mucopolysaccharidosis IIIA in humans and a-mannosidosis in mice..$^{211,214,216,217}$ In addition, there is an example in dogs. When American Staffordshire Terriers and Pit Bull Terriers with a recessively inherited, adult-onset, progressive neurodegenerative disease were found to accumulate autofluorescent storage bodies in hippocampal and cerebellar neurons, their disease was classified as an NCL. ${ }^{218}$ This classification remained unchanged with the identification of the causal genetic variant in $A R S G$, a gene with a biological function that had not yet been determined. ${ }^{219}$ Subsequently, it was shown using Arsg-knockout mice that the natural substrate for the encoded enzyme was heparan sulfate, a mucopolysaccharide, and that the storage bodies were rich in partially degraded heparan sulfate. ${ }^{220}$ Thus, the disease caused by ARSG deficiency is now considered to be a mucopolysaccharidosis. Similarly, the Shiba Inu of our case 
report were thought to have NCL until we determined that brain extracts from 1 of the dogs contained accumulated GM2 ganglioside and had a $\beta$-hexosaminidase deficiency, thus classifying the disorder as a gangliosidosis.

Ours may be the first report that storage bodies associated with GM2 gangliosidosis are autofluorescent. In fact, at least 1 report states that storage bodies from human patients with GM2 gangliosidosis do not autofluoresce. ${ }^{210}$ Thus, we examined tissue from a previously reported case of GM2 gangliosidosis in a Japanese Chin dog caused by a HEXA mutation (OMIA 001461-9615). ${ }^{209}$ As with the results reported here, the storage bodies in the tissue from the dog with the HEXA mutation were autofluorescent (Fig. 5).
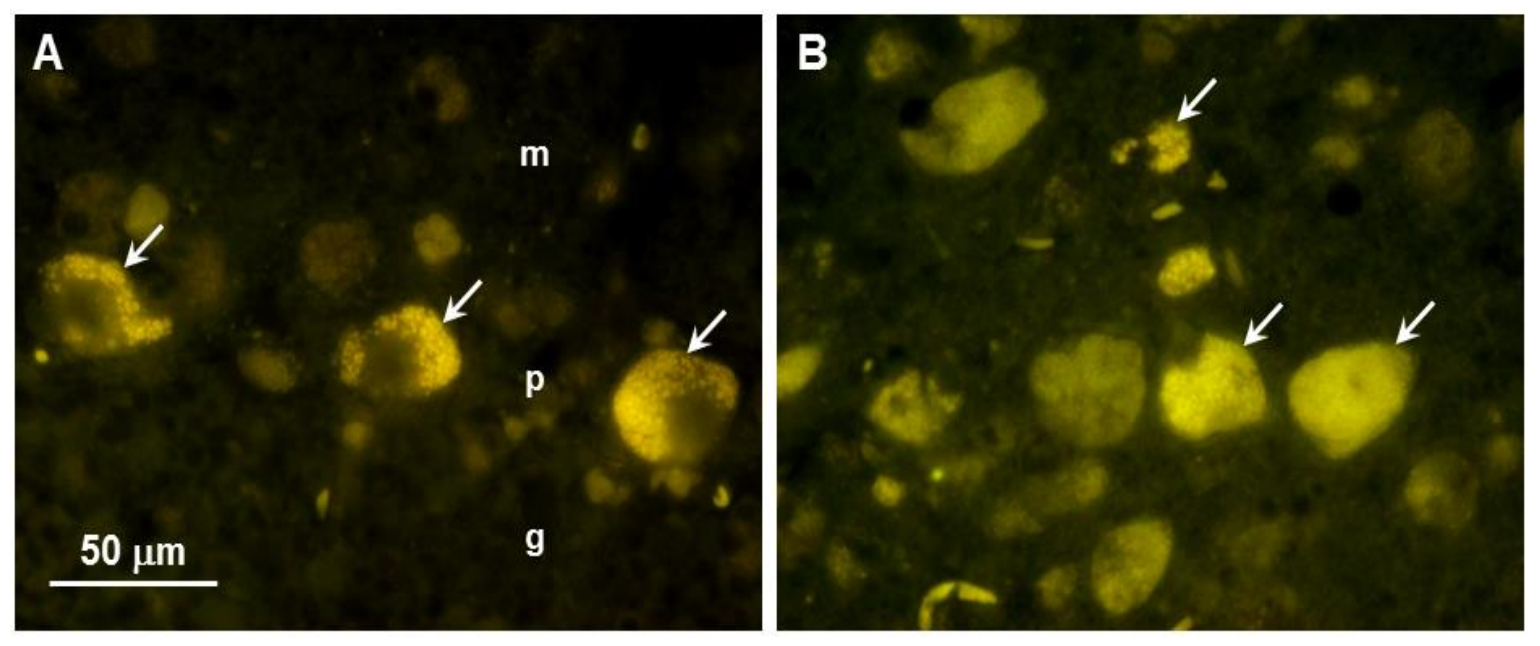

Fig. 5 Fluorescence micrographs of unstained cryostat sections of cerebellum $(\mathbf{A})$ and cerebral cortex (B) from a Japanese Chin dog with GM2 gangliosidosis due to a homozygous HEXA nonsynonymous substitution. In the cerebellum, autofluorescent inclusions were present primarily in the Purkinje cells (arrows in A) in the Purkinje cell layer ( $p$ ), although weakly fluorescent cells were also present in the molecular layer $(\mathrm{m})$. No autofluorescence was observed in the granular cell layer $(\mathrm{g})$. Neurons throughout the cerebral cortex gray matter of the parietal lobe had abundant autofluorescent inclusions (arrows in B). Bar in (A) indicates the magnification for micrographs $(\mathbf{A})$ and $(\mathbf{B})$. 


\section{Comparison with other gangliosidoses in dogs}

Although a GM1 gangliosidosis has been recognized in the Shiba Inu for $>15$ years, ${ }^{206,221,222}$ ours is the first report of a GM2 gangliosidosis in this breed. A GM2 gangliosidosis has been reported in a mixed breed dog and in members of other dog breeds including German Shorthaired Pointer, Japanese Chin, Golden Retriever, and Toy Poodle. ${ }^{209,223-227}$ The Toy Poodle, Golden Retriever and the mixed breed dog were classified as 0 variants (OMIA 001462-9615)..$^{225-227}$ The 1 bp HEXB frameshift variant responsible for the 0-variant gangliosidosis in Toy Poodles $^{228}$ is different from the $3 \mathrm{bp}$ in-frame deletion that we found in the affected Shiba Inu dogs. The molecular genetic causes for the 2 other 0 -variant gangliosidoses have not been reported.

\section{Conclusions}

Although not yet routine, the sequencing and analysis of whole genomes generated from patient DNA is becoming an increasingly important strategy for diagnosing inherited diseases in humans. ${ }^{229}$ Technical improvements in massively parallel DNA sequencing have resulted in the development of a sequencing instrument $^{\mathrm{b}}$ capable of generating deep sequence coverages of mammalian genomes for $<\$ 2,000 .{ }^{230}$ Although initially licensed exclusively for the sequencing of human genomes, ${ }^{231,232}$ this instrument can now be used to sequence domestic animal genomes. With access to this technology, it became economically feasible for us to make use of whole genome sequencing early in our efforts to identify the 
molecular genetic cause of the neurodegeneration in these Shiba Inu. Analysis of the whole genome sequence from 1 of the affected dogs identified a potentially causal candidate variant. This finding enabled us to proceed with more traditional diagnostic techniques (e.g., thin layer chromatography, enzymology, electron microscopy) that were chosen specifically to confirm or refute the causality of the candidate variant. Thus, in our case study, whole genome sequencing was an efficient and cost effective procedure that led to the identification of the Shiba Inu disease as a GM2 gangliosidosis caused by a 3 bp deletion in HEXB. Once impediments such as long turn-around times, low causal variant discovery rates and inadequate data storage facilities have been overcome, we expect whole genome sequencing to become an increasingly important tool for diagnosing heritable animal diseases.

\section{Footnotes}

aMcDonnell Genome Institute, Washington University, St Louis, MO 63108, USA

bHiSeq X Sequencing System, Illumina, Inc., San Diego, CA 92122, USA'Paw Print Genetics, 850 E. Spokane Falls Blvd. Suite 200, Spokane, WA 99202, USA

dVetGen, 3728 Plaza Drive, Suite 1, Ann Arbor, MI 48108, USA

eSigma-Aldrich, 3300 S 2nd St, St. Louis, MO 63118, USA 


\section{Supplemental Material}

\section{Whole Genome Sequence Data Processing Pipeline}

DNA from an affected Shiba Inu was submitted to a commercial sequencing centera for PCR-free library preparation and massively parallel DNA sequencing with a commercial sequencerb using paired end 150 bp reads. The sequence data went through an initial quality control, which included removing adapters using a custom Perl script and examination of overall sequence quality using FastQC (v0.11.5). Reads were trimmed based on quality values using trimmomatic (version 0.35) and then aligned to the canine reference sequence assembly CanFam3.1 using BWA (v0.7.1). The alignment files were sorted and indexed using Samtools (v1.3.1). Picard (v2.6.0) was used to merge alignment files and mark duplicate sequences by library. Base quality score recalibration, indel realignment and variant calling using HaplotypeCaller were performed using GATK (v3.6) according to best practices. Variant annotation and filtering were performed with commercial software.c Annotation was accomplished with RefSeqGenes (NCBI release 104 2016-05-23). Similarly generated whole genome sequences from dogs with other diseases $(n=102)$ or from healthy dogs $(n=3)$ served as controls.

a McDonnell Genome Institute, Washington University, St Louis, MO 63108, USA

b HiSeq X Sequencing System, Illumina, Inc., San Diego, CA 92122, USA 
Supplementary Figure 1. Alignments of $\beta$-hexosaminidase $\beta$-subunit amino acids sequences from 54 mammalian species predicted from codons surrounding the deleted Leu137 codon in the affected Shiba Inu

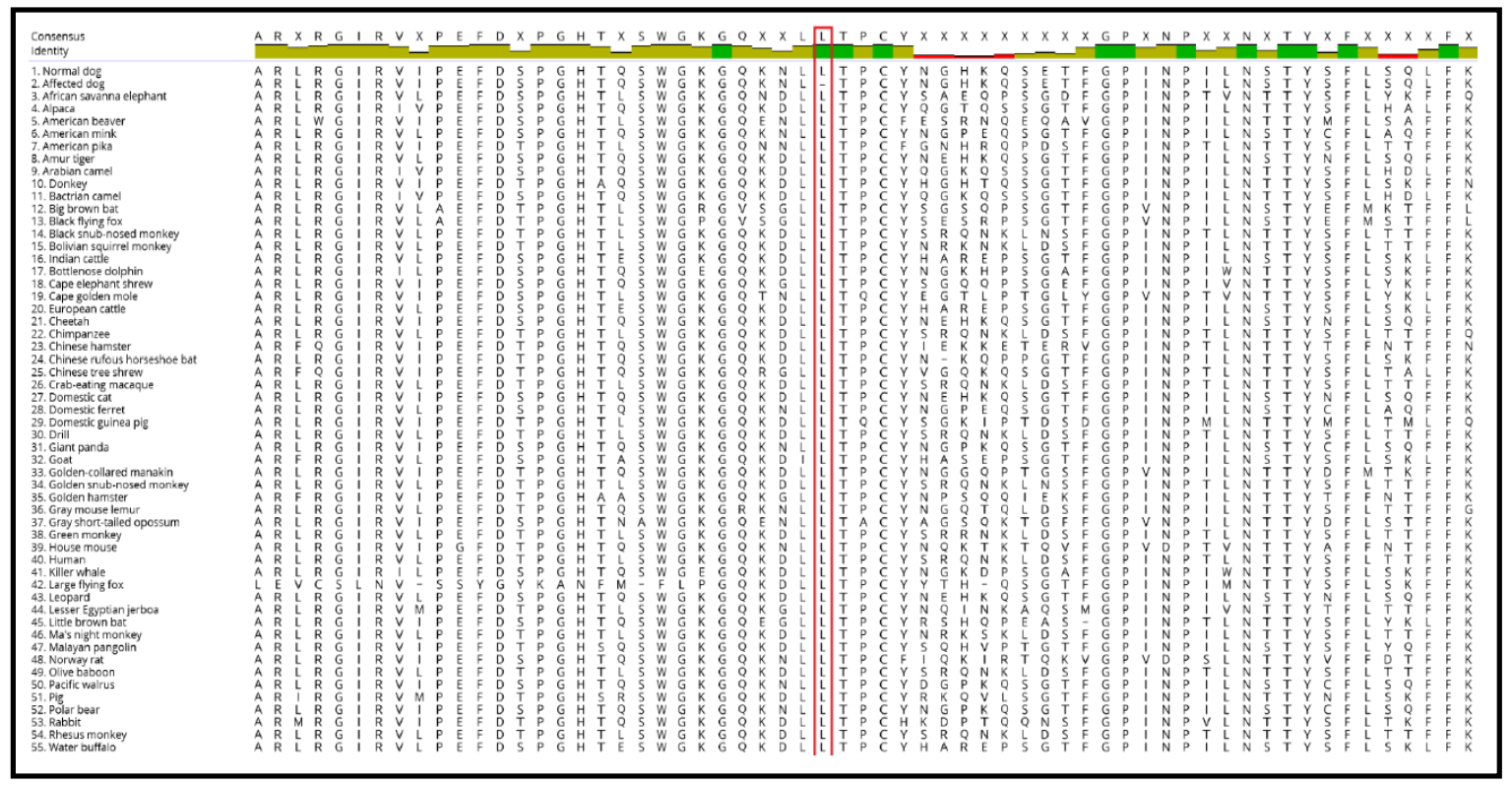

Modified from a report generated with Geneious Basic version 10.1.2 commercial software. ${ }^{1}$

${ }^{1}$ Kearse, M., Moir, R., Wilson, A., Stones-Havas, S., Cheung, M., Sturrock, S., Buxton, S., Cooper, A., Markowitz, S., Duran, C., Thierer, T., Ashton, B., Mentjies, P., \& Drummond, A. (2012). Geneious Basic: an integrated and extendable desktop software platform for the organization and analysis of sequence data. Bioinformatics, 28(12), 1647-1649. 
Supplemental Table 1: Rare variants predicted to change the primary sequence of the gene product for which the affected Shiba Inu was predicted to be homozygous

\begin{tabular}{|c|c|c|c|}
\hline Coordinate & Gene & Nucleotide change & Consequence \\
\hline $1: 924522$ & CTDP1 & c. $1990 \mathrm{C}>\mathrm{A}$ & Nonsyn SNV \\
\hline 1:104890731 & LOC106558999 & c. $-56+1$ - $-56+2$ ins T & Splicing \\
\hline $2: 33828898$ & $A D A R B 2$ & c. $1879 \mathrm{G}>\mathrm{A}$ & Nonsyn SNV \\
\hline $2: 42310546$ & SPINK5 & c. $293 \mathrm{~A}>\mathrm{T}$ & Nonsyn SNV \\
\hline $2: 43248005$ & IL6ST & c. $12 \mathrm{G}>\mathrm{T}$ & Nonsyn SNV \\
\hline $2: 48844920$ & IPO11 & c. $2096 \mathrm{G}>\mathrm{A}$ & Nonsyn SNV \\
\hline $2: 48844931$ & IPO11 & c. $2107 \mathrm{C}>\mathrm{A}$ & Nonsyn SNV \\
\hline $2: 52647551$ & CD180 & c. $646 \mathrm{C}>\mathrm{T}$ & Nonsyn SNV \\
\hline 2:57243653 & HEXB & c.945_947delCCT & Del \\
\hline $2: 58350523$ & $C C D C 113$ & c. $913 \mathrm{G}>\mathrm{A}$ & Nonsyn SNV \\
\hline $2: 58579428$ & CNGB1 & c. $214 \mathrm{C}>\mathrm{T}$ & Nonsyn SNV \\
\hline $2: 58781765$ & ADGRG3 & c. $1595 \mathrm{C}>\mathrm{T}$ & Nonsyn SNV \\
\hline $2: 65342376$ & LOC487290 & c. $203 \mathrm{G}>\mathrm{A}$ & Nonsyn SNV \\
\hline $2: 76408401$ & LACTBL1 & c. $677 \mathrm{C}>\mathrm{T}$ & Nonsyn SNV \\
\hline $3: 18314343$ & ADGRV1 & c. $3502 C>T$ & Nonsyn SNV \\
\hline 4:57953189 & SLC36A2 & c. $958 \mathrm{C}>\mathrm{T}$ & Nonsyn SNV \\
\hline 4:59883859 & SH3TC2 & c. $1273 \mathrm{G}>\mathrm{A}$ & Nonsyn SNV \\
\hline $6: 8811313$ & MUC17 & $\begin{array}{l}\text { c.1294_1295insAGACGTGACCACATCATCAGTA } \\
\text { TCTCCAGATGGGACCACATCAGCAGCTGCTC } \\
\text { CA }\end{array}$ & Stopgain \\
\hline $6: 12571677$ & TNRC18 & c. $4857 \mathrm{C}>\mathrm{G}$ & Nonsyn SNV \\
\hline $6: 13248516$ & $S D K 1$ & c. $2912 \mathrm{G}>\mathrm{T}$ & Nonsyn SNV \\
\hline 6:15276899 & ELFN1 & c. $1886 \mathrm{C}>\mathrm{T}$ & Nonsyn SNV \\
\hline 6:15413161 & TMEM184A & c. $166 \mathrm{C}>\mathrm{T}$ & Nonsyn SNV \\
\hline $6: 36540441$ & $P P L$ & c. $4405 \mathrm{C}>\mathrm{T}$ & Nonsyn SNV \\
\hline 6:36661974 & ZNF500 & c. $1361 \mathrm{G}>\mathrm{A}$ & Nonsyn SNV \\
\hline 6:39118587 & IGFALS & c. $1900 \mathrm{G}>\mathrm{A}$ & Nonsyn SNV \\
\hline 6:39121141 & NUBP2 & c. $37 \mathrm{G}>\mathrm{A}$ & Nonsyn SNV \\
\hline 6:39478018 & LOC448801 & c. $116 \mathrm{C}>\mathrm{T}$ & Nonsyn SNV \\
\hline
\end{tabular}




\begin{tabular}{|c|c|c|c|}
\hline Coordinate & Gene & Nucleotide change & Consequence \\
\hline 6:39511373 & CACNA1H & c.6328G $>T$ & Nonsyn SNV \\
\hline 6:39518309 & CACNA1H & c. $4445 G>A$ & Nonsyn SNV \\
\hline 6:39978234 & $P I G Q$ & c.292G $>T$ & Nonsyn SNV \\
\hline 6:39984223 & NHLRC4 & c. $1259 G>A$ & Nonsyn SNV \\
\hline $6: 39984362$ & NHLRC4 & c. $1120 \mathrm{G}>\mathrm{A}$ & Nonsyn SNV \\
\hline 6:39984617 & NHLRC4 & c. $865 G>A$ & Nonsyn SNV \\
\hline 6:40429609 & LOC611642 & c. $278 \mathrm{G}>\mathrm{A}$ & Nonsyn SNV \\
\hline 6:40654696 & LOC611675 & $\begin{array}{l}\text { c.993_994insCTGCCTCCATCTACCTATGTTCTT } \\
\text { AATGTATGTATACACCCCAGG }\end{array}$ & Ins \\
\hline 6:40823680 & DENND2D & c. $264 \mathrm{G}>\mathrm{T}$ & Nonsyn SNV \\
\hline 9:36330268 & CA4 & c. $412 A>G$ & Nonsyn SNV \\
\hline 11:3522330 & LOC474642 & c. $328 \mathrm{C}>\mathrm{T}$ & Stopgain \\
\hline $11: 12337210$ & SNCAIP & c. $997 \mathrm{C}>\mathrm{G}$ & Nonsyn SNV \\
\hline $14: 40359259$ & HOXA11 & c.664 665insCGG & Ins \\
\hline $14: 43195886$ & NOD1 & c. $1534 G>A$ & Nonsyn SNV \\
\hline $15: 1570206$ & HIVEP3 & c.3269C>T & Nonsyn SNV \\
\hline $15: 3345340$ & LOC100855618 & c. $1268 C>T$ & Nonsyn SNV \\
\hline $15: 5952737$ & SH3D21 & c. $308 \mathrm{G}>\mathrm{A}$ & Nonsyn SNV \\
\hline $15: 6922899$ & ZMYM1 & c.319G>T & Nonsyn SNV \\
\hline $15: 7005024$ & ZMYM6 & c. $2847 A>G$ & Nonsyn SNV \\
\hline 15:8995338 & ORC1 & c. $2554 \mathrm{G}>\mathrm{A}$ & Nonsyn SNV \\
\hline $15: 15536284$ & BEST4 & c. $479 \mathrm{C}>\mathrm{T}$ & Nonsyn SNV \\
\hline $15: 16132552$ & KLF18 & c. $1367 \mathrm{~A}>\mathrm{T}$ & Nonsyn SNV \\
\hline 15:17495861 & LOC100855686 & c. $40 \mathrm{C}>\mathrm{T}$ & Nonsyn SNV \\
\hline $15: 17763635$ & KLHL33 & c. $565 \mathrm{G}>\mathrm{C}$ & Nonsyn SNV \\
\hline $15: 18124446$ & TPPP2 & c. $479 \mathrm{G}>\mathrm{A}$ & Nonsyn SNV \\
\hline 15:22772701 & LOC106559776 & c.39_41delTGT & Del \\
\hline $15: 51916068$ & DCHS2 & c.9953C>T & Nonsyn SNV \\
\hline 16:5993505 & COR13M1 & C. $125 \mathrm{~A}>\mathrm{C}$ & Nonsyn SNV \\
\hline $16: 20282728$ & PTPRN2 & c. $1516 G>A$ & Nonsyn SNV \\
\hline
\end{tabular}




\begin{tabular}{|c|c|c|c|}
\hline Coordinate & Gene & Nucleotide change & Consequence \\
\hline 18:26154975 & LOC106559966 & c. $443 \mathrm{G}>\mathrm{C}$ & Nonsyn SNV \\
\hline 18:46124790 & LOC102153419 & c. $938 \mathrm{G}>\mathrm{A}$ & Nonsyn SNV \\
\hline 18:53442696 & LGALS12 & c.407T>C & Nonsyn SNV \\
\hline 22:52246650 & $C C D C 168$ & c. $2524 \mathrm{C}>\mathrm{T}$ & Nonsyn SNV \\
\hline 22:52356145 & ERCC5 & c. $1658 \mathrm{C}>\mathrm{T}$ & Nonsyn SNV \\
\hline 23:8212869 & $A C V R 2 B$ & c. $431 \mathrm{C}>\mathrm{T}$ & Nonsyn SNV \\
\hline 23:8790242 & TTC21A & c.782A $>C$ & Nonsyn SNV \\
\hline 23:8853006 & XIRP1 & c. $2857 \mathrm{C}>\mathrm{T}$ & Nonsyn SNV \\
\hline 24:44550537 & PPP1R3D & c.683G $>A$ & Nonsyn SNV \\
\hline $25: 35432289$ & LOC106557768 & c.556delT & Frameshift Del \\
\hline 25:35432291 & LOC106557768 & c.558_559delCC & Stopgain \\
\hline 25:37253297 & ZNF596 & c. $157 A>G$ & Nonsyn SNV \\
\hline $27: 1770147$ & PRR13 & c.344A>T & Nonsyn SNV \\
\hline $27: 2448245$ & KRT2 & c. $392 \mathrm{G}>\mathrm{C}$ & Nonsyn SNV \\
\hline 27:38938186 & $V W F$ & c.6380C>T & Nonsyn SNV \\
\hline $31: 29813863$ & C31H21orf140 & c.738_740delCTA & Del \\
\hline $31: 38348044$ & LOC100685181 & c. $241 \mathrm{G}>\mathrm{A}$ & Nonsyn SNV \\
\hline $31: 38355090$ & LOC611489 & c.568_569insCACCTCCA & Frameshift Ins \\
\hline 33:29155279 & TNK2 & c. $1597 \mathrm{G}>\mathrm{A}$ & Nonsyn SNV \\
\hline $36: 3337579$ & GALNT5 & c. $1166 A>G$ & Nonsyn SNV \\
\hline $\mathrm{X}: 1454947$ & $X G$ & c. $358 \mathrm{G}>\mathrm{A}$ & Nonsyn SNV \\
\hline $\mathrm{X}: 1459432$ & $X G$ & c. $399 \mathrm{C}>\mathrm{G}$ & Nonsyn SNV \\
\hline $\mathrm{X}: 6822143$ & WWC3 & c. $2382 \mathrm{G}>\mathrm{C}$ & Nonsyn SNV \\
\hline $\mathrm{X}: 77011557$ & TCEAL4 & c.250_270delAAGCCAGCGGAGAGCGAGGGG & Del \\
\hline
\end{tabular}




\section{Chapter 6}

\section{Discussion and Conclusion}

The previous chapters ( 2 to 5 ) presented four examples in which my thesis-related research efforts lead to reportable discoveries; however, the research described in those chapters represents only a small fraction of my entire research effort since joining the Dr. GS Johnson molecular genetics laboratory as a graduate student in 2013. This final chapter presents a more balanced picture of my overall research efforts, starting with a brief description of the research environment and available resources in the Johnson laboratory, from when my research began and up until now. Next, I present my personal experiences with different procedures for generating and interpreting the canine whole genome sequences and how they advanced during my years as a graduate student. After that, examples of strategies that were not always successful in identifying disease-causing mutations are given, as are examples of efforts to match apparently deleterious sequence variants with suspected phenotypes unrelated to the dog's primary disease. Last, are a brief description of a successful causal gene identification discovered after the writing of this thesis had already started, and suggestions to successfully conclude other ongoing canine disease investigations.

Dr. GS Johnson's molecular genetic laboratory at the University of Missouri focuses on detecting variants associated with disease phenotypes in dogs. Currently, the lab has a canine DNA repository with over 180,000 samples from multiple breeds that were accumulated over three decades of research. From 1990 
until 2010, 17 disease associated variants were identified using traditional methods, such as mapping and candidate gene exon sequencing. In 2010, NGS started to become affordable for canine disease research and since then the lab has submitted DNA samples for WGS for 96 dogs from 53 different breeds and about 40 different disease phenotypes. Together with collaborating institutes, over 500 canine WGS are available for data analysis. The causal mutations for 20 disease phenotypes have been successfully identified through WGS analysis between 2010 and now in our lab, including the four mutations discussed in chapters 2, 3, 4 and 5 (Table 1). To date, all of these successes involve recessive Mendelian traits, and most of the associated mutations are coding (exonic), with the exception of the Cane Corso PPT1 mutation, which is within a splice donor site. Most of the samples that were submitted for WGS are thought to be rare recessive Mendelian traits based on pedigree or previous knowledge (e.g. lysosomal storage diseases), with some possible exceptions, such as epilepsy, which is now thought to be a polygenic trait in most cases. 
Table 4. Successfully identified causal mutations using WGS data.

\begin{tabular}{|c|c|c|}
\hline Breed & Disease & Gene \\
\hline Chinese crested & Multiple system degeneration & SERAC1* \\
\hline Basenji & Fanconi syndrome & FAN1* \\
\hline $\begin{array}{l}\text { Soft coated wheaten } \\
\text { terrier }\end{array}$ & Paroxysmal dyskinesia & $P^{\prime G N}{ }^{80}$ \\
\hline Jack russell terrier & Spinocerebellar ataxia & KCNJ10116 \\
\hline Kerry blue terrier & Multiple system degeneration & SERAC1* \\
\hline Basenji & Progressive retinal atrophy & $S A G^{*}$ \\
\hline Chinese crested & Neuronal ceroid lipofuscinosis & MFSD8 $8^{117}$ \\
\hline Standard schnauzer & Dilated cardiomyopathy & $R B M 20^{*}$ \\
\hline Australian shepherd & Neuronal ceroid lipofuscinosis & CLN8 $8^{52}$ \\
\hline $\begin{array}{l}\text { Black russian } \\
\text { terrier/Rottweiler }\end{array}$ & Laryngeal paralysis/spongiform encephalopaty & $\begin{array}{l}\text { RAB3GAP123 } \\
3,234\end{array}$ \\
\hline English cocker spaniel & Peripheral neuropathy & STK11IP* \\
\hline Golden retriever & Neuronal ceroid lipofuscinosis & CLN5 $5^{143}$ \\
\hline Australian cattle dog & Neuronal ceroid lipofuscinosis & CLN5 82 \\
\hline Cane corso & Neuronal ceroid lipofuscinosis & $P P T 153$ \\
\hline Tibetan terrier & Progressive retinal atrophy & $F A M 161 A^{235}$ \\
\hline Shiba inu & GM2 gangliosidosis & $H E X B^{*}$ \\
\hline Dachshund & Methemoglobinemia & CYB5R3* $^{*}$ \\
\hline Mixed breed & Methemoglobinemia & CYB5R3* $^{*}$ \\
\hline Ns duck tolling retriever & Degenerative encephalopathy & $R B 1 C C 1^{*}$ \\
\hline Mixed breed & Beta-mannosidosis & $M A N B A^{*}$ \\
\hline
\end{tabular}

${ }^{*}$ manuscripts on different stages of completion 
There have been significant differences in the quality of sequence data generated at different points in time that result from to changes in sequencing chemistry and instrumentation. Prior to 2016, the University of Missouri sequences were generated using an Illumina HiSeq 2500 which could generate up to 1 TB of data in six days when using the high-output mode and $300 \mathrm{~GB}$ when using the rapid run mode. The paired read lengths of Hiseq 2500 varied between 2x 50bp to 2x 125bp, and the cost per sample was $\sim \$ 5,000$ for a $30 \mathrm{X}$ average coverage of a $\sim 2.6 \mathrm{~GB}$ genome. From 2016, the Illumina HiSeq X Ten series became available for use with non-human samples. This new technology is almost four times faster it can produce 3.6 TB in six days at a reduced cost of $\sim \$ 2,000$ per sample (30X coverage). Hiseq $X$ Ten improved the sequencing chemistry and technology, decreasing the error rates and slightly increased the read lengths (2x 150bp). There are also differences in the library preparation methodologies between the older and more current sequences. The earlier sequences (2010-2013) used PCRbased libraries with fragment lengths of $300 \mathrm{bp}$ and $400 \mathrm{bp}$. Between 2013 and 2015, kits became available to produce PCR-free libraries with fragment lengths of $350 \mathrm{bp}$ and $550 \mathrm{bp}$.

PCR-based library preparation generally consists of fragmenting the DNA molecules, fusing the fragments to adapters, followed by amplification. The DNA fragmentation step were achieved by either mechanical force (sonication, nebulization or acoustic shearing) or by enzymatic digestion (non-specific endonuclease cocktails). The ends of the fragments were enzymatically repaired and the adapter sequences were ligated to each end. The fragments of the desired 
size can be selected by either gel extraction, or by using solid-phase reversible immobilization (SPRI) beads. The amplification to enrich the product that has the adapters ligated to both ends is then achieved by PCR cycles and the sample is ready for the sequencing by synthesis. ${ }^{236,237}$ PCR-free protocols require a higher concentration of input DNA than the PCR methodology, and use kits such as the Illumina TruSeq DNA PCR-free kit that include DNA fragmentation, fragment size selection and final library quantification in their protocols.

Slightly different PCR-free library preparations were used for the newer HiSeq X Ten sequences, where the fragment lengths are limited to $350 \mathrm{bp}$ or $450 \mathrm{bp}$, because of the physical limitations of the new, bead-dependent ordered array flowcells that are used in this technology. The newer flow cells have much more surface area for cluster generation, which is how the HiSeq X Ten can generate so much more data than the earlier machines. Each flow cell and sequencing technology supports an optimal insert size. Smaller insert sizes amplify more efficiently, however there is a risk of generating a lower number of reads and coverage, and if a much larger insert size is sequenced, there is a possibility of overloading the clustering and bridge amplification step, causing a high number of duplicates, sequencing overlapping, cross-contamination of multiplexed samples and index hoping (mislabeling of reads).

As mentioned in Chapter 1, library preparation is an important step that can introduce biases to the sequences and variation calling. The difference was very noticeable especially between the PCR libraries and the PCR-free libraries. The PCR-based libraries had many sequencing gaps and inconsistent coverage (zero 
to 33X) along the genome, especially along high GC\% and other repetitive regions. High GC\% regions require a higher melting point, due to the strong hydrogen bonds, and often times have low primer specificity. In addition, PCR artifacts, including amplification bias, mispriming events, and higher number of duplicate sequences, resulted in many false positive variants that could not be later verified with Sanger sequencing. Many of the false positives were within the first exons, which can be explained by the high GC\% content of those regions. Sometimes those false positive variants and lower coverage occurred within functionally relevant genes for the diseases that were being researched at the time, and were considered plausible candidates until disproved by Sanger sequencing. Since the lab started to use PCR-free library based sequences, most of the single nucleotide candidate variants reported in the WGS could be later verified through other molecular methods.

Improvements in the computational resources and bioinformatics pipelines were also accomplished over time. Initially, the computational resources were more limited and fewer samples could be processed simultaneously. The entire data processing (including trimming, QC, alignment and variant calling) with the older available computational resources could take from one and a half week to two weeks per sample. Because the canine lab is not the only one that generates a high number of WGS at the University of Missouri, it could take a few months before a variant report was available and annotated. A "Biocomputer cluster" was developed as a part of the 2016 Cyberinfrastructure Plan Update at the University of Missouri (https://mospace.umsystem.edu/xmlui/handle/10355/48334). The 
cluster is currently available and consists of 37 Dell R630 Broadwell nodes with 28 cores, 512 GB of RAM, and 3TB of local SSD storage. The expanded HPC resources have the potential to process over 30 individuals per day with the current QC and variant calling pipeline.

Several algorithms were developed overtime to analyze sequencing data, and they can be broadly classified in different categories and subcategories. For example, preprocessing and quality control software include adapter trimmers, duplicate read removal tools, error correction, variant recalibration, and other algorithms. Data analysis algorithms include read aligners, variant callers, copy number variant callers, annotators and visualization tools. One of the reasons for the "forest of software" in the bioinformatics field is because many laboratories develop their own algorithms optimized for their own data and biological question. Some of those software packages are no longer maintained or updated, hence checking the dates of the latest update and frequencies of the updates is important. For this reason, there are efforts to curate the available and up to date bioinformatics software in one place, such as (OMICtools $)^{238}$. Best practices and user friendly pipelines for whole genome sequence analysis were developed by different institutions and companies, with the goal of helping biologists with little or no computational background. Some developed freely available software, such as the Genome Analysis Toolkit (Broad institute) ${ }^{239}$ and Galaxy ${ }^{240}$, and some others offer commercially available software, such as Softgenetics NextGene ${ }^{\mathrm{TM}}$, Geneious ${ }^{241}$, CLCbio Genomics Workbench ${ }^{242}$, Golden Helix SNP and Variation Suite ${ }^{\mathrm{TM}}$, among others. Some of these software attempt to integrate algorithms pertaining to each 
different WGS analysis step into just one user friendly interface, such as the one from Softgenetics NextGene ${ }^{\mathrm{TM}}$.

The bioinformatics pipeline used within the Johnson lab from 2010 until early 2015 was mostly done by in-house perl scripts for adapter trimming and quality control, and the NextGene software was used for aligning the reads to a reference sequence, variant calling, annotation and visualization. The variants were filtered and stored in a PostgreSQL database. The downside of the NextGene software, other than the cost of the software license, is that the file format of the output is specific to NextGene and extra steps that can add a few days to the processing time are required to convert the files to a more universal format, such as BAM, if the use of different analysis software is desired. In my experience, the file conversion sometimes resulted in corrupted and unusable BAM files. The time used for the troubleshooting such file conversion errors could be used for other more productive analysis.

As increasingly more individual sequences become available, a joint analysis is recommended. Joint analysis uses the evidence from multiple samples to determine the genotypes of all samples, calling the variants occurring in sites with lower coverage with a much higher accuracy and sensitivity than individual variant calling. ${ }^{239}$ However, joint analysis also introduced a problem called the "N+1" problem. ${ }^{239}$ Joint analysis requires that all samples are run together in one batch because not all >100 samples were generated at the same time, whenever a new sample was introduced, the whole batch variant calling needed to be re-run from scratch. As expected, the more samples that are added to the batch, the more 
computationally intensive it becomes to generate the variant calls, resulting in a scalability problem. These problems were addressed by the genome analysis toolkit (GATK) best practices pipeline with the introduction of the GVCF (genomic variant call files) workflow ${ }^{239}$. The GVCF files are similar to regular variant call files (VCF). The difference is that GVCFs contain a record of every position in the genome, or the selected interval, regardless of whether a variant was detected at that site or not. For example, when a newer sample with a certain number of "new" variants and "new" coordinates that were not previously called on the older samples is added to the cohort, the GVCF of the older samples already contains information about each sample's probable genotype in those "new" coordinates, unlike the regular VCF, thus, GVCFs save time and computational power when doing the joint analysis. Since individual GVCF files are generated and stored for each sample as a first step, the joint analysis can be done at any time, using any cohort of samples as desired.

Once the variant calling is done, the next step for detecting causal mutations is variant filtering and annotation. There are two main annotation sources, NCBI (Refseq) and Ensembl. Both sources use different procedures for their gene predictions: Refseq is a collection of non-redundant curated mRNA models, and Ensembl is a database that contains gene models from multiple sources mapped into the reference genome. Therefore, filtering variants based on those two annotation sources can result in very different variant classifications. From the end of 2015, our lab purchased a lab license for the Golden Helix SNP and Variation Suite $^{\mathrm{TM}}$ (SVS), and this software has a user-friendly interface for annotating and 
filtering variants. The variant call files of 120 dogs were annotated with the SVS software using both, Ensembl and RefSeq sources. The total number of variants in this cohort that were classified as potentially functional (variants that are near or within exons and are non-synonymous) was 115,614 . The variants that were common to both $\mathrm{NCBI}$ and Ensembl annotation sources was 50,599 (43.8\%) however $21,715(18.8 \%)$ were only detected by the Ensembl annotation and $43,300(47.5 \%)$ were detected only by the NCBI annotation. In chapter 5 , the causal mutation on HEXB was almost missed because only the Ensembl annotation was interrogated at the time, and the causal deletion was placed in the "unknown classification" bin based on that annotation. Using both annotation sources is important, and even then the causal variants could still be misclassified and be filtered out of the final variant reports, as seen on Table 2. The two instances in Table 2 where the mutations were not present on either annotation report are the mutations on the FAN1 and PPT1 genes. Both of those mutations were detected through the visual inspection of the alignments using a genome browser, like IGV. ${ }^{204,243}$ Those genes in particular were visually inspected because of previous genome mapping studies (FAN1) or because of known previous associations between human orthologs and human diseases with similar phenotypes (PPT1). The FAN1 mutation was probably absent from the reports not because of an annotation error, but because the causal mutation is a large deletion and those types of variants are not reported by the regular variant calling algorithms. Large insertions, deletions, inversions and duplications require specific structural variant (SV) callers that are not yet well established in our pipeline. The 
PPT1 mutation is a splicing mutation, and splicing mutations have major annotation discrepancy problems. In order to predict splice site effects, an accurate exon-intron boundary must be annotated in a gene, and complete transcript information is helpful, if not essential. Some genes have multiple isoforms and it is difficult to known which isoform is active in the cell type of interest. Also, there are overlapping genes that might share an exon, or the variant falls in the exon of one of the genes and in the intron, splice-site, promoter or regulatory region for the other gene. Even genes with only one isoform might harbor variants with more than one consequence, and all of those issues must be considered when evaluating candidate mutations. ${ }^{244}$ 
Table 5. List of genes that were successfully associated to a disease phenotype by Dr. GS Johnson's lab, and which annotation source correctly predicted the variant effect and included the variant in the final mutation report.

\begin{tabular}{|l|c|c|c|c|}
\hline Causal Gene & Ensembl & NCBI & Both & None $^{*}$ \\
\hline SERAC1a & & & $\mathbf{X}$ & \\
\hline FAN1 & & & & $\mathbf{X}$ \\
\hline PIGN & & & $\mathbf{X}$ & \\
\hline KCNJ10 & & & $\mathbf{X}$ & \\
\hline SERAC1b & & & $\mathbf{X}$ & \\
\hline SAG & & & $\mathbf{X}$ & \\
\hline MFSD8 & & & $\mathbf{X}$ & \\
\hline RBM20 & & & $\mathbf{X}$ & \\
\hline CLN8 & & & $\mathbf{X}$ & \\
\hline RAB3GAP1 & & & $\mathbf{X}$ & \\
\hline STK11IP & & & $\mathbf{X}$ & \\
\hline CLN5a & & & & \\
\hline CLN5b & & & & \\
\hline PPT1 & & & & \\
\hline FAM161A & & & & \\
\hline HEXB & & & & \\
\hline CYB5R3a & & & & \\
\hline CYB5R3b & & & & \\
\hline
\end{tabular}

So far our successes in finding disease-causing mutations were done either by direct candidate gene scanning or rare variant filtering. The direct candidate gene scanning approach is used when there is previous knowledge of genes that are 
associated with a particular disease phenotype. This methodology was previously mentioned and described with the FAN1, CLN5 (Chapter 3) and PPT1 (Chapter 4) examples. Visually inspecting a candidate gene approach does not necessarily require a variant report. The visualization of the reads aligned to the reference sequence is done simply by loading a BAM file, the reference sequence (FASTA file) and the annotation sources (GFF or other file formats) into a genome browser. Any potential deleterious mutations near or within the exons of the biologically known candidate genes are then noted and further tested for phenotypic association. When there are hundreds of possible candidate genes, all the variants occurring within those genes can be filtered in the variant reports, rather than visually inspecting thousands of exons. This approach could be used to find potential risk factors for epilepsy, for example. Sometimes the mutation is within a gene that was never associated to the specific disease phenotype before, which was the case for the PIGN mutation (Chapter 2). In this case, one affected individual had 418 unique mutations in comparison to 100 control dogs. Since none of the 302 genes harboring these mutations had a clear biological association to the paroxysmal dyskinesia, a second affected dog was sequenced and the only variant that was common to both affected dogs and was not present in the other 100 control dogs was the PIGN:c.398C>T substitution. Unfortunately, the approach of sequencing more than one affected dog and filtering the rare variants has not yet been successful for the identification of the causal mutations of some other diseases, such as the standard poodle polymicrogyria (PMG), border terrier canine epileptoid cramping syndrome (CECS), border terrier dysmyelinogenisis, 
gordon setter "darned unnamed new genetic disease" (DUNGd), Jack Russel terrier neonatal granuloprival ataxia and bouvier des Fladres glaucoma. There are many possible reasons why the approach has not yet worked for those cases. First, unlike the paroxysmal dyskinesia cases where only one mutation was unique to both affected individuals within the cohort, these other cases have several unique variants, where most have no obvious biological correlation, and are yet to be tested for phenotype-genotype association. Also, there is a chance that the chosen affected samples do not have the same disease and are "phenocopies" Furthermore, as mentioned before, the variant reports do not contain information about structural and copy number variants. There could be possible annotation errors or incomplete annotation, especially involving the regulatory regions within introns or even intergenic. Other types of variants that are being underexplored at the moment are mutations in $\mathrm{CpG}$ islands that could cause loss of imprinting, or other epigenetic effects.

Some of those diseases also have GWAS data generated as an attempt to narrow down the locus of the causal mutation. A few candidate variants within the associated regions are currently under investigation for many of these diseases, including a structural change within an intron that could potentially disrupt a splice site on FIG4 in Gordon Setters with DUNGd. Recently the lab acquired the Agena MassArray system that could potentially genotype up to 30 variants at once. However, so far our experience with designing synergetic and consistent assays has been disappointing. Once the technical difficulties of dealing with the 
equipment and technology are addressed, we might be able to "kiss multiple frogs at once" and more efficiently discover disease-associated genotypes.

There are other ways to analyze and filter variants that can potentially lead to the discovery of new genotype-phenotype relationships, such as incidental findings and "reverse genetics". Incidental findings happen in situations where you find a deleterious mutation in a gene known to cause a phenotype that is different than the disease in the original research plan. For example, a dog that was sequenced because of a heart defect that also has a deleterious mutation in a gene that is known to cause a late onset eye disease. Reverse genetics can be defined as the process of determining which phenotypes arise as a result of a particular variant. ${ }^{245}$ Reverse genetics using WGS could be accomplished by identifying individuals that are heterozygous for a deleterious mutation that could potentially cause disease in the homozygous state, and testing multiple individuals of the same family or breed until a homozygous mutant can be found to verify that the expected particular disease phenotype occurs in this individual. Those two methodologies can be hard to achieve because they may require a very detailed clinical follow up and the cooperation of the sample providers is required, which unfortunately is not always achievable.

Collaboration with veterinary clinicians and an accurate characterization of the disease is very important for different steps of the research, including the process for choosing which samples to sequence. In chapter 5 , the HEXB mutation causing GM2 gangliosidosis could have been discovered by directly sequencing the HEXB gene and save sequencing money, if an enzymatic assay had been performed by 
the clinicians. However, this example also showed that WGS can be useful for personalized veterinary medicine as it can correctly provide a diagnosis.

During my graduate studies I have analyzed 8 different GWAS and tested over 35 different candidate variants that were not associated with the expected disease phenotype. Of the 8 GWAS, one was done in collaboration with the University of Pennsylvania, where I identified a locus on chromosome 9 associated to increased susceptibility with Mycobaterium avium complex (MAC) in Miniature Standard Schnauzers. Recently, researchers at University of Philadelphia identified the phenotype-associated mutation, a homozygous codon deletion in the CARD9 gene that is present within the locus and was highlighted through my analysis. More recently, I detected a single nucleotide variant in the MANBA gene in a mixed breed dog that was suspected of having a lysosomal storage disease, consistent with a diagnosis of $\beta$-mannosidosis. Enzymatic analysis has confirmed this diagnosis.

With the current and future improvements optimization of the variant discovery pipeline, the mutation analysis will become faster and easier. Traits that are not Mendelian are still very challenging and could also explain why we only have a $\sim 30 \%$ discovery success rate. The current optimized variant calling algorithms are not able to detect large deletions, insertions, inversions, insertions of LINEs or SINEs and copy number variations, which can also potentially explain some of the disease causing variations. Also, it still hard to determine the impact of intronic, 5'UTR, 3'-UTR and other regulatory variants with the current canine genome annotation, and as discussed earlier, we are perhaps still missing many causal 
variants due to annotation errors, reference sequence gaps or other data generation or analysis biases. Sequencing technologies with longer reads are already available but too expensive for many routine uses. In the future they will probably cost much less and they will add a lot of information concerning structural differences among individual and cohort samples. There is a current international and multi-collaborative effort to test many of the open source structural variant (SV) and copy number variant (CNV) calling software packages in over 500 canine genomes. The aim is to better characterize the canine genome and potentially detect disease causing variations that are not reported by the GATK or platypus variant callers. In order to identify potential risk factors for complex traits such as epilepsy, different strategies will be required as the risk alleles might be common in the general population, but will only have effect in individuals that have a burden of other risk alleles.

There are still many improvements to be made, but WGS analysis is without doubt a very efficient methodology for detecting disease-causing mutations. The potential conjunction of WGS with other molecular methods such as ATAC-seq for identifying active chromatin and regulatory portions of the genome in the cell type of interest, and CRISPR/Cas9 for assisting in gene function discovery, is very exciting. Improvements in sequencing technology and analytic tools will lead to interesting discoveries that help our best friends to be healthier and happier. 


\section{References}

1. Ember CR, Ember M. Encyclopedia of medical anthropology : health and illness in the world's cultures. New York: Kluwer Academic/Plenum Publishers; 2004.

2. Lamarck JBPAdMd. Histoire naturelle des animaux sans vertèbres : présentant les caractères généraux et particuliers de ces animaux ... : précédée d'une introduction offrant la détermination des caractères essentiels de l'animal, sa distinction du végétal et des autres corps naturels, enfin, l'exposition des principes fondamentaux de la zoologie. Paris: Verdière; 1815.

3. Wang Y, Liu H, Sun Z. Lamarck rises from his grave: parental environment-induced epigenetic inheritance in model organisms and humans. Biol Rev Camb Philos Soc 2017.

4. Darwin C. On the origin of species by means of natural selection, or, The preservation of favoured races in the struggle for life, 5th thousand. ed. London,: J. Murray; 1860;ix, 1 , 502 p.

5. Mendel G, Fisher RA. Experiments in plant hybridisation. Edinburgh,: Oliver \& Boyd; 1965;ix, $95 \mathrm{p}$.

6. Gayon J. From Mendel to epigenetics: History of genetics. C R Biol 2016;339:225-230.

7. Dahm R. Friedrich Miescher and the discovery of DNA. Dev Biol 2005;278:274-288.

8. Miescher F. Ueber die chemische Zusammensetzung der Eiterzellen. Medicinisch-chemische Untersuchungen 1871;4:441-460.

9. Mccarty M. A Retrospective Look at the Discovery of the Genetic Role of DNA. Faseb J 1994;8:889-890.

10. Pray L. Discovery of DNA structure and function: Watson and Crick. Nature Education 2008;1:100.

11. Watson JD, Crick FH. The structure of DNA. Cold Spring Harb Symp Quant Biol 1953;18:123131. 
12. Levene PA. On the Chemistry of the Chromatin Substance of the Nerve Cell. J Med Res $1903 ; 10: 204-211$.

13. Chargaff E, Vischer E. Nucleoproteins, nucleic acids, and related substances. Annu Rev Biochem 1948;17:201-226.

14. Chargaff E, Magasanik B, et al. The nucleotide composition of ribonucleic acids. J Am Chem Soc 1949;71:1513.

15. RajBhandary UL, Kohrer C. Early days of tRNA research: discovery, function, purification and sequence analysis. J Biosci 2006;31:439-451.

16. Sanger F, Donelson JE, Coulson AR, et al. Use of DNA polymerase I primed by a synthetic oligonucleotide to determine a nucleotide sequence in phage fI DNA. Proc Natl Acad Sci U S A 1973;70:1209-1213.

17. Maxam AM, Gilbert W. A new method for sequencing DNA. Proc Natl Acad Sci U S A 1977;74:560-564.

18. Kulski JK. Next-Generation Sequencing - An Overview of the History, Tools, and "Omic" Applications. 2016.

19. Saiki RK, Gelfand DH, Stoffel S, et al. Primer-directed enzymatic amplification of DNA with a thermostable DNA polymerase. Science 1988;239:487-491.

20. Barba M, Czosnek H, Hadidi A. Historical perspective, development and applications of nextgeneration sequencing in plant virology. Viruses 2014;6:106-136.

21. Franca LT, Carrilho E, Kist TB. A review of DNA sequencing techniques. Q Rev Biophys 2002;35:169-200.

22. Heather JM, Chain B. The sequence of sequencers: The history of sequencing DNA. Genomics 2016;107:1-8. 
23. Dewey FE, Pan S, Wheeler MT, et al. DNA sequencing: clinical applications of new DNA sequencing technologies. Circulation 2012;125:931-944.

24. Consortium IHGS. Initial sequencing and analysis of the human genome. Nature 2001;409.

25. Venter JC, Adams MD, Myers EW, et al. The sequence of the human genome. Science 2001;291:1304-1351.

26. Waterston $\mathrm{RH}$, Lander ES, Sulston JE. On the sequencing of the human genome. Proc Natl Acad Sci U S A 2002;99:3712-3716.

27. Koepfli KP, Paten B, Genome KCoS, et al. The Genome 10K Project: a way forward. Annu Rev Anim Biosci 2015;3:57-111.

28. Genome KCOS. Genome 10K: a proposal to obtain whole-genome sequence for 10,000 vertebrate species. J Hered 2009;100:659-674.

29. Stephens ZD, Lee SY, Faghri F, et al. Big Data: Astronomical or Genomical? PLoS Biol 2015;13:e1002195.

30. van Steenbeek FG, Hytonen MK, Leegwater PA, et al. The canine era: the rise of a biomedical model. Anim Genet 2016;47:519-527.

31. Wang GD, Zhai W, Yang HC, et al. The genomics of selection in dogs and the parallel evolution between dogs and humans. Nat Commun 2013;4:1860.

32. Schoenebeck JJ, Ostrander EA. The genetics of canine skull shape variation. Genetics 2013;193:317-325.

33. Boyko AR. The domestic dog: man's best friend in the genomic era. Genome Biol $2011 ; 12: 216$

34. Freedman AH, Lohmueller KE, Wayne RK. Evolutionary History, Selective Sweeps, and Deleterious Variation in the Dog. Annual Review of Ecology, Evolution, and Systematics 2016;47:73-96. 
35. Fan Z, Silva P, Gronau I, et al. Worldwide patterns of genomic variation and admixture in gray wolves. Genome Res 2016;26:163-173.

36. Skoglund P, Ersmark E, Palkopoulou E, et al. Ancient wolf genome reveals an early divergence of domestic dog ancestors and admixture into high-latitude breeds. Curr Biol $2015 ; 25: 1515-1519$.

37. Reiter T, Jagoda E, Capellini TD. Dietary Variation and Evolution of Gene Copy Number among Dog Breeds. PLoS One 2016;11:e0148899.

38. vonHoldt BM, Pollinger JP, Earl DA, et al. A genome-wide perspective on the evolutionary history of enigmatic wolf-like canids. Genome Res 2011;21:1294-1305.

39. Lindblad-Toh $\mathrm{K}$, Wade CM, Mikkelsen TS, et al. Genome sequence, comparative analysis and haplotype structure of the domestic dog. Nature 2005;438:803-819.

40. Kirkness EF, Bafna V, Halpern AL, et al. The dog genome: survey sequencing and comparative analysis. Science 2003;301:1898-1903.

41. Ostrander EA, Lindblad-Toh K, Lander ES, et al. Sequencing the Genome of the Domestic Dog Canis famililaris. Grant proposal.

42. Batzoglou S, Jaffe DB, Stanley K, et al. ARACHNE: a whole-genome shotgun assembler. Genome Res 2002;12:177-189.

43. Hoeppner MP, Lundquist A, Pirun M, et al. An improved canine genome and a comprehensive catalogue of coding genes and non-coding transcripts. PLoS One 2014;9:e91172. 44. Mellersh CS, Langston AA, Acland GM, et al. A linkage map of the canine genome. Genomics 1997;46:326-336.

45. Yuzbasiyan-Gurkan V, Blanton $\mathrm{SH}, \mathrm{Cao} \mathrm{Y}$, et al. Linkage of a microsatellite marker to the canine copper toxicosis locus in Bedlington terriers. Am J Vet Res 1997;58:23-27. 
46. Lohi H, Young EJ, Fitzmaurice SN, et al. Expanded repeat in canine epilepsy. Science 2005;307:81.

47. Voorbij AM, van Steenbeek FG, Vos-Loohuis M, et al. A contracted DNA repeat in LHX3 intron 5 is associated with aberrant splicing and pituitary dwarfism in German shepherd dogs. PLoS One 2011;6:e27940.

48. Karlsson EK, Baranowska I, Wade CM, et al. Efficient mapping of mendelian traits in dogs through genome-wide association. Nat Genet 2007;39:1321-1328.

49. Cadieu E, Neff MW, Quignon P, et al. Coat variation in the domestic dog is governed by variants in three genes. Science 2009;326:150-153.

50. Awano T, Johnson GS, Wade CM, et al. Genome-wide association analysis reveals a SOD1 mutation in canine degenerative myelopathy that resembles amyotrophic lateral sclerosis. Proc Natl Acad Sci U S A 2009;106:2794-2799.

51. Faller KM, Bras J, Sharpe SJ, et al. The Chihuahua dog: A new animal model for neuronal ceroid lipofuscinosis CLN7 disease? J Neurosci Res 2016;94:339-347.

52. Guo J, Johnson GS, Brown HA, et al. A CLN8 nonsense mutation in the whole genome sequence of a mixed breed dog with neuronal ceroid lipofuscinosis and Australian Shepherd ancestry. Mol Genet Metab 2014;112:302-309.

53. Kolicheski A, Barnes Heller HL, Arnold S, et al. Homozygous PPT1 Splice Donor Mutation in a Cane Corso Dog With Neuronal Ceroid Lipofuscinosis. J Vet Intern Med 2017;31:149-157.

54. Hirz M, Drogemuller M, Schanzer A, et al. Neuronal ceroid lipofuscinosis (NCL) is caused by the entire deletion of CLN8 in the Alpenlandische Dachsbracke dog. Mol Genet Metab 2017;120:269-277.

55. Niskanen J, Dillard K, Arumilli M, et al. Nonsense variant in COL7A1 causes recessive dystrophic epidermolysis bullosa in Central Asian Shepherd dogs. PLoS One 2017;12:e0177527. 
56. Bentley DR, Balasubramanian S, Swerdlow HP, et al. Accurate whole human genome sequencing using reversible terminator chemistry. Nature 2008;456:53-59.

57. Rothberg JM, Hinz W, Rearick TM, et al. An integrated semiconductor device enabling nonoptical genome sequencing. Nature 2011;475:348-352.

58. Eid J, Fehr A, Gray J, et al. Real-time DNA sequencing from single polymerase molecules. Science 2009;323:133-138.

59. Mardis ER. A decade's perspective on DNA sequencing technology. Nature 2011;470:198203.

60. Illumina. An introduction to Next-Generation Sequencing Technology. wwwilluminacom/technology/next-generation-sequencinghtml 2016.

61. Horner DS, Pavesi G, Castrignano T, et al. Bioinformatics approaches for genomics and post genomics applications of next-generation sequencing. Brief Bioinform 2010;11:181-197.

62. Doyle K. DNA Quantitation in Next-Generation Sequencing Library Workflows. Promega Corporation Website http://wwwpromegacom/resources/pubhub/dna-quantitation-in-nextgeneration-sequencing-library-workflows/ 2015.

63. Aird D, Ross MG, Chen WS, et al. Analyzing and minimizing PCR amplification bias in Illumina sequencing libraries. Genome Biol 2011;12:R18.

64. McCall CM, Mosier S, Thiess M, et al. False positives in multiplex PCR-based next-generation sequencing have unique signatures. J Mol Diagn 2014;16:541-549.

65. Ross MG, Russ $C$, Costello $M$, et al. Characterizing and measuring bias in sequence data. Genome Biol 2013;14:R51.

66. Illumina. Quality Scores for Next-Generation Sequencing: Assessing sequencing accuracy using Phred quality scoring. Technical Note: Sequencing 2011. 
67. Endrullat C, Glokler J, Franke P, et al. Standardization and quality management in nextgeneration sequencing. Appl Transl Genom 2016;10:2-9.

68. Taylor JC, Martin HC, Lise S, et al. Factors influencing success of clinical genome sequencing across a broad spectrum of disorders. Nat Genet 2015;47:717-726.

69. Choi M, Scholl UI, Ji W, et al. Genetic diagnosis by whole exome capture and massively parallel DNA sequencing. Proc Natl Acad Sci U S A 2009;106:19096-19101.

70. Bolze A, Byun M, McDonald D, et al. Whole-exome-sequencing-based discovery of human FADD deficiency. Am J Hum Genet 2010;87:873-881.

71. Worthey EA, Mayer AN, Syverson GD, et al. Making a definitive diagnosis: successful clinical application of whole exome sequencing in a child with intractable inflammatory bowel disease. Genet Med 2011;13:255-262.

72. Manolio TA, Collins FS, Cox NJ, et al. Finding the missing heritability of complex diseases. Nature 2009;461:747-753.

73. Manolio TA. Genomewide association studies and assessment of the risk of disease. N Engl J Med 2010;363:166-176.

74. Kingsmore SF, Saunders CJ. Deep sequencing of patient genomes for disease diagnosis: when will it become routine? Sci Transl Med 2011;3:87ps23.

75. Green ED, Guyer MS, National Human Genome Research I. Charting a course for genomic medicine from base pairs to bedside. Nature 2011;470:204-213.

76. Roychowdhury S, lyer MK, Robinson DR, et al. Personalized oncology through integrative high-throughput sequencing: a pilot study. Sci Transl Med 2011;3:111ra121.

77. Pleasance ED, Cheetham RK, Stephens PJ, et al. A comprehensive catalogue of somatic mutations from a human cancer genome. Nature 2010;463:191-196. 
78. Snyder TM, Khush KK, Valantine HA, et al. Universal noninvasive detection of solid organ transplant rejection. Proc Natl Acad Sci U S A 2011;108:6229-6234.

79. Palomaki GE, Kloza EM, Lambert-Messerlian GM, et al. DNA sequencing of maternal plasma to detect Down syndrome: an international clinical validation study. Genet Med 2011;13:913920.

80. Kolicheski AL, Johnson GS, Mhlanga-Mutangadura T, et al. A homozygous PIGN missense mutation in Soft-Coated Wheaten Terriers with a canine paroxysmal dyskinesia. Neurogenetics 2017;18:39-47.

81. Livak KJ. Allelic discrimination using fluorogenic probes and the 5' nuclease assay. Genetic Analysis:Biomolecular Engineering 1999;14:143-149.

82. Kolicheski A, Johnson GS, O'Brien DP, et al. Australian Cattle Dogs with Neuronal Ceroid Lipofuscinosis are Homozygous for a CLN5 Nonsense Mutation Previously Identified in Border Collies. J Vet Intern Med 2016;30:1149-1158.

83. Demirkiran M, Jankovic J. Paroxysmal dyskinesias: clinical features and classification. Ann Neurol 1995;38:571-579.

84. Bhatia KP. Paroxysmal dyskinesias. Mov Disord 2011;26:1157-1165.

85. Jankovic J, Demirkiran M. Classification of paroxysmal dyskinesias and ataxias. Adv Neurol 2002;89:387-400.

86. Waln O, Jankovic J. Paroxysmal movement disorders. Neurol Clin 2015;33:137-152.

87. Erro R, Sheerin UM, Bhatia KP. Paroxysmal dyskinesias revisited: a review of 500 genetically proven cases and a new classification. Mov Disord 2014;29:1108-1116.

88. Bruno MK, Lee HY, Auburger GW, et al. Genotype-phenotype correlation of paroxysmal nonkinesigenic dyskinesia. Neurology 2007;68:1782-1789. 
89. Bruno MK, Hallett M, Gwinn-Hardy K, et al. Clinical evaluation of idiopathic paroxysmal kinesigenic dyskinesia: new diagnostic criteria. Neurology 2004;63:2280-2287.

90. Blakeley J, Jankovic J. Secondary paroxysmal dyskinesias. Mov Disord 2002;17:726-734.

91. Chen WJ, Lin $Y$, Xiong ZQ, et al. Exome sequencing identifies truncating mutations in PRRT2 that cause paroxysmal kinesigenic dyskinesia. Nat Genet 2011;43:1252-1255.

92. Rainier S, Thomas D, Tokarz D, et al. Myofibrillogenesis regulator 1 gene mutations cause paroxysmal dystonic choreoathetosis. Arch Neurol 2004;61:1025-1029.

93. Suls A, Dedeken P, Goffin K, et al. Paroxysmal exercise-induced dyskinesia and epilepsy is due to mutations in SLC2A1, encoding the glucose transporter GLUT1. Brain 2008;131:18311844.

94. Wang JL, Cao L, Li XH, et al. Identification of PRRT2 as the causative gene of paroxysmal kinesigenic dyskinesias. Brain 2011;134:3493-3501.

95. Lee $H Y, X u Y$, Huang $Y$, et al. The gene for paroxysmal non-kinesigenic dyskinesia encodes an enzyme in a stress response pathway. Hum Mol Genet 2004;13:3161-3170.

96. Brockmann K. The expanding phenotype of GLUT1-deficiency syndrome. Brain Dev 2009;31:545-552.

97. Anand G, Padeniya A, Hanrahan D, et al. Milder phenotypes of glucose transporter type 1 deficiency syndrome. Dev Med Child Neurol 2011;53:664-668.

98. Schneider SA, Paisan-Ruiz C, Garcia-Gorostiaga I, et al. GLUT1 gene mutations cause sporadic paroxysmal exercise-induced dyskinesias. Mov Disord 2009;24:1684-1688.

99. Weber YG, Storch A, Wuttke TV, et al. GLUT1 mutations are a cause of paroxysmal exertioninduced dyskinesias and induce hemolytic anemia by a cation leak. J Clin Invest 2008;118:21572168. 
100. Li M, Niu F, Zhu X, et al. PRRT2 Mutant Leads to Dysfunction of Glutamate Signaling. Int J Mol Sci 2015;16:9134-9151.

101. Nobile C, Striano P. PRRT2: a major cause of infantile epilepsy and other paroxysmal disorders of childhood. Prog Brain Res 2014;213:141-158.

102. Shen $Y$, Ge WP, Li Y, et al. Protein mutated in paroxysmal dyskinesia interacts with the active zone protein RIM and suppresses synaptic vesicle exocytosis. Proc Natl Acad Sci U S A $2015 ; 112: 2935-2941$.

103. Du W, Bautista JF, Yang H, et al. Calcium-sensitive potassium channelopathy in human epilepsy and paroxysmal movement disorder. Nat Genet 2005;37:733-738.

104. Castiglioni C, Verrigni D, Okuma C, et al. Pyruvate dehydrogenase deficiency presenting as isolated paroxysmal exercise induced dystonia successfully reversed with thiamine supplementation. Case report and mini-review. Eur J Paediatr Neurol 2015;19:497-503.

105. Urkasemsin G, Olby NJ. Canine paroxysmal movement disorders. Vet Clin North Am Small Anim Pract 2014;44:1091-1102.

106. Penderis J, Franklin RJ. Dyskinesia in an adult bichon frise. J Small Anim Pract 2001;42:2425.

107. Black V, Garosi L, Lowrie M, et al. Phenotypic characterisation of canine epileptoid cramping syndrome in the Border terrier. J Small Anim Pract 2014;55:102-107.

108. Herrtage ME, Palmer AC. Episodic falling in the cavalier King Charles spaniel. Vet Rec 1983;112:458-459.

109. Packer RA, Patterson EE, Taylor JF, et al. Characterization and mode of inheritance of a paroxysmal dyskinesia in Chinook dogs. J Vet Intern Med 2010;24:1305-1313.

110. Wolf M, Bruehschwein A, Sauter-Louis C, et al. An inherited episodic head tremor syndrome in Doberman pinscher dogs. Mov Disord 2011;26:2381-2386. 
111. Guevar J, De Decker S, Van Ham LM, et al. Idiopathic head tremor in English bulldogs. Mov Disord 2014;29:191-194.

112. Meyers KM, Lund JE, Padgett G, et al. Hyperkinetic episodes in Scottish Terrier dogs. J Am Vet Med Assoc 1969;155:129-133.

113. Shelton GD. Muscle pain, cramps and hypertonicity. Vet Clin North Am Small Anim Pract 2004;34:1483-1496.

114. Forman OP, Penderis J, Hartley C, et al. Parallel mapping and simultaneous sequencing reveals deletions in BCAN and FAM83H associated with discrete inherited disorders in a domestic dog breed. PLoS Genet 2012;8:e1002462.

115. Gill JL, Tsai KL, Krey C, et al. A canine BCAN microdeletion associated with episodic falling syndrome. Neurobiol Dis 2012;45:130-136.

116. Gilliam D, O'Brien DP, Coates JR, et al. A homozygous KCNJ10 mutation in Jack Russell Terriers and related breeds with spinocerebellar ataxia with myokymia, seizures, or both. J Vet Intern Med 2014;28:871-877.

117. Guo J, O'Brien DP, Mhlanga-Mutangadura T, et al. A rare homozygous MFSD8 single-basepair deletion and frameshift in the whole genome sequence of a Chinese Crested dog with neuronal ceroid lipofuscinosis. BMC Vet Res 2015;10:960.

118. Ohba C, Okamoto N, Murakami Y, et al. PIGN mutations cause congenital anomalies, developmental delay, hypotonia, epilepsy, and progressive cerebellar atrophy. Neurogenetics 2014;15:85-92.

119. Kinoshita T. Biosynthesis and deficiencies of glycosylphosphatidylinositol. Proceedings of the Japan Academy, Series B 2014;90:130-143.

120. Sanger TD, Delgado MR, Gaebler-Spira D, et al. Classification and definition of disorders causing hypertonia in childhood. Pediatrics 2003;111:e89-97. 
121. Sanger TD, Chen D, Fehlings DL, et al. Definition and classification of hyperkinetic movements in childhood. Mov Disord 2010;25:1538-1549.

122. Bhatia KP, Soland VL, Bhatt MH, et al. Paroxysmal exercise-induced dystonia: eight new sporadic cases and a review of the literature. Mov Disord 1997;12:1007-1012.

123. Hooser SB, VR B. Methylxanthine (chocolate and caffeine toxicosis). . Philadelphia: 1986;pp. 191-192.

124. Kinoshita T, Fujita M, Maeda Y. Biosynthesis, remodelling and functions of mammalian GPIanchored proteins: recent progress. J Biochem 2008;144:287-294.

125. Orlean P, Menon AK. Thematic review series: lipid posttranslational modifications. GPI anchoring of protein in yeast and mammalian cells, or: how we learned to stop worrying and love glycophospholipids. J Lipid Res 2007;48:993-1011.

126. Gaynor EC, Mondesert G, Grimme SJ, et al. MCD4 encodes a conserved endoplasmic reticulum membrane protein essential for glycosylphosphatidylinositol anchor synthesis in yeast. Mol Biol Cell 1999;10:627-648.

127. Hong $Y$, Maeda $Y$, Watanabe R, et al. Pig-n, a mammalian homologue of yeast Mcd4p, is involved in transferring phosphoethanolamine to the first mannose of the glycosylphosphatidylinositol. J Biol Chem 1999;274:35099-35106.

128. Vainauskas S, Menon AK. Ethanolamine phosphate linked to the first mannose residue of glycosylphosphatidylinositol (GPI) lipids is a major feature of the GPI structure that is recognized by human GPI transamidase. J Biol Chem 2006;281:38358-38364.

129. Khayat M, Tilghman JM, Chervinsky I, et al. A PIGN mutation responsible for multiple congenital anomalies-hypotonia-seizures syndrome 1 (MCAHS1) in an Israeli-Arab family. Am J Med Genet A 2016;170A:176-182. 
130. Nakagawa T, Taniguchi-lkeda M, Murakami Y, et al. A novel PIGN mutation and prenatal diagnosis of inherited glycosylphosphatidylinositol deficiency. Am J Med Genet A 2016;170A:183-188.

131. Maydan G, Noyman I, Har-Zahav A, et al. Multiple congenital anomalies-hypotonia-seizures syndrome is caused by a mutation in PIGN. J Med Genet 2011;48:383-389.

132. McKean DM, Niswander L. Defects in GPI biosynthesis perturb Cripto signaling during forebrain development in two new mouse models of holoprosencephaly. Biol Open 2012;1:874883.

133. Brady PD, Moerman P, De Catte $L$, et al. Exome sequencing identifies a recessive PIGN splice site mutation as a cause of syndromic congenital diaphragmatic hernia. Eur J Med Genet 2014;57:487-493.

134. Couser NL, Masood MM, Strande NT, et al. The phenotype of multiple congenital anomalies-hypotonia-seizures syndrome 1: report and review. Am J Med Genet A 2015;167A:2176-2181.

135. Fleming L, Lemmon M, Beck N, et al. Genotype-phenotype correlation of congenital anomalies in multiple congenital anomalies hypotonia seizures syndrome (MCAHS1)/PIGNrelated epilepsy. Am J Med Genet A 2016;170A:77-86.

136. Groffen AJ, Klapwijk T, van Rootselaar AF, et al. Genetic and phenotypic heterogeneity in sporadic and familial forms of paroxysmal dyskinesia. J Neurol 2013;260:93-99.

137. Mole SE, Cotman SL. Genetics of the neuronal ceroid lipofuscinoses (Batten disease). Biochim Biophys Acta 2015;1852:2237-2241.

138. Jolly RD, Palmer DN. The neuronal ceroid-lipofuscinoses (Batten disease): comparative aspects. Neuropathol Appl Neurobiol 1995;21:50-60. 
139. Anderson GW, Goebel HH, Simonati A. Human pathology in NCL. Biochim Biophys Acta 2013;1832:1807-1826.

140. Sanders DN, Farias FH, Johnson GS, et al. A mutation in canine PPT1 causes early onset neuronal ceroid lipofuscinosis in a Dachshund. Mol Genet Metab 2010;100:349-356.

141. Awano T, Katz ML, O'Brien DP, et al. A frame shift mutation in canine TPP1 (the ortholog of human CLN2) in a juvenile Dachshund with neuronal ceroid lipofuscinosis. Mol Genet Metab 2006;89:254-260.

142. Melville SA, Wilson CL, Chiang CS, et al. A mutation in canine CLN5 causes neuronal ceroid lipofuscinosis in Border collie dogs. Genomics 2005;86:287-294.

143. Gilliam D, Kolicheski A, Johnson GS, et al. Golden Retriever dogs with neuronal ceroid lipofuscinosis have a two-base-pair deletion and frameshift in CLN5. Mol Genet Metab 2015;115:101-109.

144. Katz ML, Farias FH, Sanders DN, et al. A missense mutation in canine CLN6 in an Australian shepherd with neuronal ceroid lipofuscinosis. J Biomed Biotechnol 2011;2011:198042.

145. Katz ML, Khan S, Awano T, et al. A mutation in the CLN8 gene in English Setter dogs with neuronal ceroid-lipofuscinosis. Biochem Biophys Res Commun 2005;327:541-547.

146. Awano T, Katz ML, O'Brien DP, et al. A mutation in the cathepsin D gene (CTSD) in American Bulldogs with neuronal ceroid lipofuscinosis. Mol Genet Metab 2006;87:341-348.

147. Farias $\mathrm{FH}$, Zeng $\mathrm{R}$, Johnson GS, et al. A truncating mutation in ATP13A2 is responsible for adult-onset neuronal ceroid lipofuscinosis in Tibetan terriers. Neurobiol Dis 2011;42:468-474. 148. Wohlke A, Philipp U, Bock P, et al. A one base pair deletion in the canine ATP13A2 gene causes exon skipping and late-onset neuronal ceroid lipofuscinosis in the Tibetan terrier. PLoS Genet 2011;7:e1002304. 
149. Cho DY, Leipold HW, Rudolph R. Neuronal ceroidosis (ceroid-lipofuscinosis) in a Blue Heeler dog. Acta Neuropathol 1986;69:161-164.

150. Nimmo Wilkie JS, Hudson EB. Neuronal and generalized ceroid-lipofuscinosis in a cocker spaniel. Vet Pathol 1982;19:623-628.

151. Goebel HH, Bilzer T, Dahme E, et al. Morphological studies in canine (Dalmatian) neuronal ceroid-lipofuscinosis. Am J Med Genet Suppl 1988;5:127-139.

152. Rossmeisl JHJ, Duncan R, Fox J, et al. Neuronal ceroid-lipofuscinosis in a Labrador Retriever. J Vet Diagn Invest 2003;15:457-460.

153. Jolly RD, Sutton RH, Smith RI, et al. Ceroid-lipofuscinosis in miniature Schnauzer dogs. Aust Vet J 1997;75:67.

154. Narfstrom K, Wrigstad A. Clinical, electrophysiological, and morphological findings in a case of neuronal ceroid lipofuscinosis in The Polish Owczarek Nizinny (PON) dog. Vet Q 1995;17:46. 155. Appleby EC, Longstaffe JA. Ceroid-lipofuscinosis in two saluki dogs. JComp Path 1982;92. 156. Zeng R, Coates JR, Johnson GC, et al. Breed distribution of SOD1 alleles previously associated with canine degenerative myelopathy. J Vet Intern Med 2014;28:515-521.

157. Li H, Durbin R. Fast and accurate short read alignment with Burrows-Wheeler transform. Bioinformatics 2009;25:1754-1760.

158. Li H, Handsaker B, Wysoker A, et al. The Sequence Alignment/Map format and SAMtools. Bioinformatics 2009;25:2078-2079.

159. Rimmer A, Phan H, Mathieson I, et al. Integrating mapping-, assembly- and haplotypebased approaches for calling variants in clinical sequencing applications. Nat Genet 2014;46:912918.

160. Rama Rao KV, Kielian T. Astrocytes and lysosomal storage diseases. Neuroscience 2016;323:195-206. 
161. Wood PA, Sisk DB, Styer E, et al. Animal model: ceroidosis (ceroid-lipofuscinosis) in Australian cattle dogs. Am J Med Genet 1987;26:891-898.

162. Sisk DB, Levesque DC, Wood PA, et al. Clinical and pathologic features of ceroid lipofuscinosis in two Australian cattle dogs. J Am Vet Med Assoc 1990;197:361-364.

163. Taylor RM, Farrow BR. Ceroid lipofuscinosis in the border collie dog: retinal lesions in an animal model of juvenile Batten disease. Am J Med Genet 1992;42:622-627.

164. Taylor RM, Farrow BR. Ceroid-lipofuscinosis in border collie dogs. Acta Neuropathol $1988 ; 75: 627-631$.

165. Studdert VP, Mitten RW. Clinical features of ceroid lipofuscinosis in border collie dogs. Aust Vet J 1991;68:137-140.

166. Franks JN, Dewey CW, Walker MA, et al. Computed tomographic findings of ceroid lipofuscinosis in a dog. J Am Anim Hosp Assoc 1999;35:430-435.

167. Mizukami K, Kawamichi T, Koie H, et al. Neuronal ceroid lipofuscinosis in Border Collie dogs in Japan: clinical and molecular epidemiological study (2000-2011). ScientificWorldJournal 2012;2012:383174.

168. Mizukami K, Chang HS, Yabuki A, et al. Novel rapid genotyping assays for neuronal ceroid lipofuscinosis in Border Collie dogs and high frequency of the mutant allele in Japan. J Vet Diagn Invest 2011;23:1131-1139.

169. Savukoski M, Klockars T, Holmberg V, et al. CLN5, a novel gene encoding a putative transmembrane protein mutated in Finnish variant late infantile neuronal ceroid lipofuscinosis. Nat Genet 1998;19:286-288.

170. Xin W, Mullen TE, Kiely R, et al. CLN5 mutations are frequent in juvenile and late-onset non-Finnish patients with NCL. Neurology 2010;74:565-571. 
171. Kousi M, Lehesjoki AE, Mole SE. Update of the mutation spectrum and clinical correlations of over 360 mutations in eight genes that underlie the neuronal ceroid lipofuscinoses. Hum Mutat 2012;33:42-63.

172. Mancini C, Nassani S, Guo Y, et al. Adult-onset autosomal recessive ataxia associated with neuronal ceroid lipofuscinosis type 5 gene (CLN5) mutations. J Neurol 2015;262:173-178.

173. Carcel-Trullols J, Kovacs AD, Pearce DA. Cell biology of the NCL proteins: What they do and don't do. Biochim Biophys Acta 2015;1852:2242-2255.

174. Kopra O, Vesa J, von Schantz C, et al. A mouse model for Finnish variant late infantile neuronal ceroid lipofuscinosis, CLN5, reveals neuropathology associated with early aging. Hum Mol Genet 2004;13:2893-2906.

175. Frugier T, Mitchell NL, Tammen I, et al. A new large animal model of CLN5 neuronal ceroid lipofuscinosis in Borderdale sheep is caused by a nucleotide substitution at a consensus splice site (c.571+1G>A) leading to excision of exon 3. Neurobiol Dis 2008;29:306-315.

176. Amorim IS, Mitchell NL, Palmer DN, et al. Molecular neuropathology of the synapse in sheep with CLN5 Batten disease. Brain Behav 2015;5:e00401.

177. Hughes SM, Hope KM, Xu JB, et al. Inhibition of storage pathology in prenatal CLN5deficient sheep neural cultures by lentiviral gene therapy. Neurobiol Dis 2014;62:543-550. 178. Perentos N, Martins AQ, Watson TC, et al. Translational neurophysiology in sheep: measuring sleep and neurological dysfunction in CLN5 Batten disease affected sheep. Brain $2015 ; 138: 862-874$.

179. Palmer DN, Neverman NJ, Chen JZ, et al. Recent studies of ovine neuronal ceroid lipofuscinoses from BARN, the Batten Animal Research Network. Biochim Biophys Acta $2015 ; 1852: 2279-2286$. 
180. Bradbury AM, Gurda BL, Casal ML, et al. A review of gene therapy in canine and feline models of lysosomal storage disorders. Hum Gene Ther Clin Dev 2015;26:27-37.

181. Katz ML, Coates JR, Sibigtroth CM, et al. Enzyme replacement therapy attenuates disease progression in a canine model of late-infantile neuronal ceroid lipofuscinosis (CLN2 disease). J Neurosci Res 2014;92:1591-1598.

182. Vuillemenot BR, Kennedy D, Cooper JD, et al. Nonclinical evaluation of CNS-administered TPP1 enzyme replacement in canine CLN2 neuronal ceroid lipofuscinosis. Mol Genet Metab $2015 ; 114: 281-293$.

183. Katz ML, Tecedor $\mathrm{L}$, Chen $\mathrm{Y}$, et al. AAV gene transfer delays disease onset in a TPP1deficient canine model of the late infantile form of Batten disease. Sci Transl Med 2015;7:313ra180.

184. Weleber RG, Gupta N, Trzupek KM, et al. Electroretinographic and clinicopathologic correlations of retinal dysfunction in infantile neuronal ceroid lipofuscinosis (infantile Batten disease). Mol Genet Metab 2004;83:128-137.

185. Burset M, Seledtsov IA, Solovyev VV. Analysis of canonical and non-canonical splice sites in mammalian genomes. Nucleic Acids Res 2000;28:4364-4375.

186. Krawczak M, Thomas NS, Hundrieser B, et al. Single base-pair substitutions in exon-intron junctions of human genes: nature, distribution, and consequences for mRNA splicing. Hum Mutat 2007;28:150-158.

187. Baralle D, Baralle M. Splicing in action: assessing disease causing sequence changes. J Med Genet 2005;42:737-748.

188. Yeo G, Burge CB. Maximum entropy modeling of short sequence motifs with applications to RNA splicing signals. J Comput Biol 2004;11:377-394. 
189. Popp MW, Maquat LE. Organizing principles of mammalian nonsense-mediated mRNA decay. Annu Rev Genet 2013;47:139-165.

190. Vesa J, Hellsten E, Verkruyse LA, et al. Mutations in the palmitoyl protein thioesterase gene causing infantile neuronal ceroid lipofuscinosis. Nature 1995;376:584-587.

191. Gupta P, Soyombo AA, Atashband A, et al. Disruption of PPT1 or PPT2 causes neuronal ceroid lipofuscinosis in knockout mice. Proc Natl Acad Sci U S A 2001;98:13566-13571.

192. Jalanko A, Vesa J, Manninen T, et al. Mice with Ppt1Deltaex4 mutation replicate the INCL phenotype and show an inflammation-associated loss of interneurons. Neurobiol Dis 2005;18:226-241.

193. Bouchelion A, Zhang Z, Li Y, et al. Mice homozygous for c.451C>T mutation in Cln1 gene recapitulate INCL phenotype. Ann Clin TransI Neurol 2014;1:1006-1023.

194. Miller JN, Kovacs AD, Pearce DA. The novel Cln1(R151X) mouse model of infantile neuronal ceroid lipofuscinosis (INCL) for testing nonsense suppression therapy. Hum Mol Genet 2015;24:185-196.

195. Sarkar C, Chandra G, Peng S, et al. Neuroprotection and lifespan extension in Ppt1(-/-) mice by NtBuHA: therapeutic implications for INCL. Nat Neurosci 2013;16:1608-1617.

196. Finn R, Kovacs AD, Pearce DA. Treatment of the Ppt1(-/-) mouse model of infantile neuronal ceroid lipofuscinosis with the N-methyl-D-aspartate (NMDA) receptor antagonist memantine. J Child Neurol 2013;28:1159-1168.

197. Lu JY, Nelvagal HR, Wang L, et al. Intrathecal enzyme replacement therapy improves motor function and survival in a preclinical mouse model of infantile neuronal ceroid lipofuscinosis. Mol Genet Metab 2015;116:98-105. 
198. Thada V, Miller JN, Kovacs AD, et al. Tissue-specific variation in nonsense mutant transcript level and drug-induced read-through efficiency in the CIn1(R151X) mouse model of INCL. J Cell Mol Med 2016;20:381-385.

199. Tracy CJ, Sanders DN, Bryan JN, et al. Intravitreal Implantation of Genetically Modified Autologous Bone Marrow-Derived Stem Cells for Treating Retinal Disorders. Adv Exp Med Biol 2016;854:571-577.

200. Griffey MA, Wozniak D, Wong M, et al. CNS-directed AAV2-mediated gene therapy ameliorates functional deficits in a murine model of infantile neuronal ceroid lipofuscinosis. Mol Ther 2006;13:538-547.

201. Aungaroon G, Hallinan B, Jain P, et al. Correlation Among Genotype, Phenotype, and Histology in Neuronal Ceroid Lipofuscinoses: An Individual Patient Data Meta-Analysis. Pediatr Neurol 2016.

202. Evans J, Katz ML, Levesque D, et al. A variant form of neuronal ceroid lipofuscinosis in American bulldogs. J Vet Intern Med 2005;19:44-51.

203. Bolger AM, Lohse M, Usadel B. Trimmomatic: a flexible trimmer for Illumina sequence data. Bioinformatics 2014;30:2114-2120.

204. Robinson JT, Thorvaldsdottir H, Winckler W, et al. Integrative genomics viewer. Nat Biotechnol 2011;29:24-26.

205. Mowjoodi A, Paton TA, Scherer SW. Discrimination of SNPs in GC-rich regions using a modified hydrolysis probe chemistry protocol. Biotechniques 2014;57:313-316.

206. Yamato O, Endoh D, Kobayashi A, et al. A novel mutation in the gene for canine acid betagalactosidase that causes GM1-gangliosidosis in Shiba dogs. J Inherit Metab Dis 2002;25:525526. 
207. Lawson CA, Martin DR. Animal models of GM2 gangliosidosis: utility and limitations. Appl Clin Genet 2016;9:111-120.

208. Sandhoff K, Harzer K. Gangliosides and gangliosidoses: principles of molecular and metabolic pathogenesis. J Neurosci 2013;33:10195-10208.

209. Sanders DN, Zeng R, Wenger DA, et al. GM2 gangliosidosis associated with a HEXA missense mutation in Japanese Chin dogs: a potential model for Tay Sachs disease. Mol Genet Metab 2013;108:70-75.

210. Brownstein S, Carpenter S, Polomeno RC, et al. Sandhoff's disease (GM2 gangliosidosis type 2). Histopathology and ultrastructure of the eye. Arch Ophthalmol 1980;98:1089-1097. 211. Lowden JA, Callahan JW, Gravel RA, et al. Type 2 GM1 gangliosidosis with long survival and neuronal ceroid lipofuscinosis. Neurology 1981;31:719-724.

212. Fontaine G, Resibois A, Tondeur M, et al. Gangliosidosis with total hexosaminidase deficiency: clinical, biochemical and ultrastructural studies and comparison with conventional cases of Tay-Sachs disease. Acta Neuropathol 1973;23:118-132.

213. Suzuki K. Neuropathology of late onset gangliosidoses. A review. Dev Neurosci 1991;13:205-210.

214. Oldfors A, Sourander P. Storage of lipofuscin in neurons in mucopolysaccharidosis. Report on a case of Sanfilippo's syndrome with histochemical and electron-microscopic findings. Acta Neuropathol 1981;54:287-292.

215. Radke J, Stenzel W, Goebel HH. Human NCL Neuropathology. Biochim Biophys Acta 2015;1852:2262-2266.

216. Wisniewski K, Rudelli R, Laure-Kamionowska M, et al. Sanfilippo disease, type A with some features of ceroid lipofuscinosis. Neuropediatrics 1985;16:98-105. 
217. Damme M, Stroobants S, Walkley SU, et al. Cerebellar alterations and gait defects as therapeutic outcome measures for enzyme replacement therapy in alpha-mannosidosis. J Neuropathol Exp Neurol 2011;70:83-94.

218. Siso S, Navarro C, Hanzlicek D, et al. Adult onset thalamocerebellar degeneration in dogs associated to neuronal storage of ceroid lipopigment. Acta Neuropathol 2004;108:386-392. 219. Abitbol M, Thibaud JL, Olby NJ, et al. A canine Arylsulfatase G (ARSG) mutation leading to a sulfatase deficiency is associated with neuronal ceroid lipofuscinosis. Proc Natl Acad Sci U S A 2010;107:14775-14780.

220. Kowalewski B, Lamanna WC, Lawrence R, et al. Arylsulfatase G inactivation causes loss of heparan sulfate 3-O-sulfatase activity and mucopolysaccharidosis in mice. Proc Natl Acad Sci U S A 2012;109:10310-10315.

221. Yamato O, Ochiai K, Masuoka Y, et al. GM1 gangliosidosis in shiba dogs. Vet Rec 2000;146:493-496.

222. Yamato O, Masuoka Y, Yonemura M, et al. Clinical and clinico-pathologic characteristics of Shiba dogs with a deficiency of lysosomal acid beta-galactosidase: a canine model of human GM1 gangliosidosis. J Vet Med Sci 2003;65:213-217.

223. Karbe E, Schiefer B. Familial amaurotic idiocy in male German shorthair pointers. Pathol Vet 1967;4:223-232.

224. Cummings JF, Wood PA, Walkley SU, et al. GM2 gangliosidosis in a Japanese spaniel. Acta Neuropathol 1985;67:247-253.

225. Yamato O, Matsuki N, Satoh H, et al. Sandhoff disease in a golden retriever dog. J Inherit Metab Dis 2002;25:319-320.

226. Tamura S, Tamura Y, Uchida K, et al. GM2 gangliosidosis variant 0 (Sandhoff-like disease) in a family of toy poodles. J Vet Intern Med 2010;24:1013-1019. 
227. Kohyama M, Yabuki A, Kawasaki Y, et al. GM2 Gangliosidosis Variant 0 (Sandhoff Disease) in a Mixed-Breed Dog. J Am Anim Hosp Assoc 2015;51:396-400.

228. Rahman MM, Chang HS, Mizukami K, et al. A frameshift mutation in the canine HEXB gene in toy poodles with GM2 gangliosidosis variant 0 (Sandhoff disease). Vet J 2012;194:412-416. 229. Precone V, Del Monaco V, Esposito MV, et al. Cracking the Code of Human Diseases Using Next-Generation Sequencing: Applications, Challenges, and Perspectives. Biomed Res Int 2015;2015:161648.

230. Park ST, Kim J. Trends in Next-Generation Sequencing and a New Era for Whole Genome Sequencing. Int Neurourol J 2016;20:S76-83.

231. Hall N. It's only human. Genome Biol 2014;15:104.

232. Watson M. Illuminating the future of DNA sequencing. Genome Biol 2014;15:108.

233. Mhlanga-Mutangadura T, Johnson GS, Schnabel RD, et al. A mutation in the Warburg syndrome gene, RAB3GAP1, causes a similar syndrome with polyneuropathy and neuronal vacuolation in Black Russian Terrier dogs. Neurobiol Dis 2016;86:75-85.

234. Mhlanga-Mutangadura T, Johnson GS, Ashwini A, et al. A Homozygous

RAB3GAP1:c.743delC Mutation in Rottweilers with Neuronal Vacuolation and Spinocerebellar Degeneration. J Vet Intern Med 2016;30:813-818.

235. Downs LM, Mellersh CS. An Intronic SINE insertion in FAM161A that causes exon-skipping is associated with progressive retinal atrophy in Tibetan Spaniels and Tibetan Terriers. PLoS One 2014;9:e93990.

236. van Dijk EL, Jaszczyszyn Y, Thermes C. Library preparation methods for next-generation sequencing: tone down the bias. Exp Cell Res 2014;322:12-20.

237. Head SR, Komori HK, LaMere SA, et al. Library construction for next-generation sequencing: overviews and challenges. Biotechniques 2014;56:61-64, 66, 68, passim. 
238. Henry VJ, Bandrowski AE, Pepin AS, et al. OMICtools: an informative directory for multiomic data analysis. Database (Oxford) 2014;2014.

239. McKenna A, Hanna M, Banks E, et al. The Genome Analysis Toolkit: a MapReduce framework for analyzing next-generation DNA sequencing data. Genome Res 2010;20:12971303.

240. Afgan E, Baker D, van den Beek M, et al. The Galaxy platform for accessible, reproducible and collaborative biomedical analyses: 2016 update. Nucleic Acids Res 2016;44:W3-W10.

241. Kearse M, Moir R, Wilson A, et al. Geneious Basic: an integrated and extendable desktop software platform for the organization and analysis of sequence data. Bioinformatics 2012;28:1647-1649.

242. CLC Genomics Workbench 9.5.3. In. https://www.qiagenbioinformatics.com/.

243. Thorvaldsdottir H, Robinson JT, Mesirov JP. Integrative Genomics Viewer (IGV): highperformance genomics data visualization and exploration. Brief Bioinform 2013;14:178-192. 244. McCarthy DJ, Humburg P, Kanapin A, et al. Choice of transcripts and software has a large effect on variant annotation. Genome Med 2014;6:26.

245. Griffiths AJF MJ, Suzuki DT, et al. . An Introduction to Genetic Analysis., 7th edition ed. Available from: https://www.ncbi.nlm.nih.gov/books/NBK21843/: New York: W. H. Freeman; 2000. 


\section{VITA}

Ana Leticia Kolicheski was born in 1985 in the city Curitiba, capital of the state of Parana (PR) in Brazil. She obtained her Bachelor's in Veterinary Medicine at the Universidade Federal do Parana (UFPR) in December 2009, Curitiba, PR, and her two Master's degree in Animal Breeding and Genetics were completed at Wageningen University (WU), Wageningen, in The Netherlands, and at the Swedish University of Agricultural Sciences (SLU), Uppsala, Sweden in 2012. She started her PhD studies at the University of Missouri in February of 2013 and completed her PhD in July of 2017. 